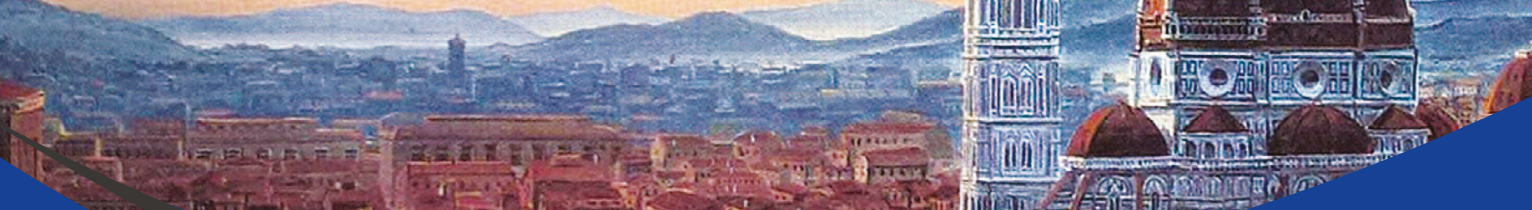

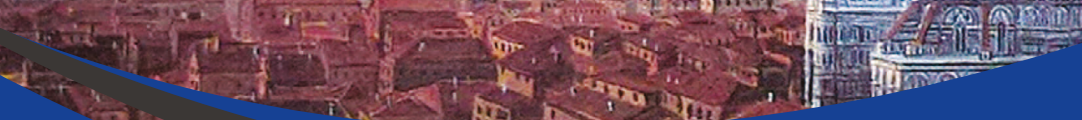
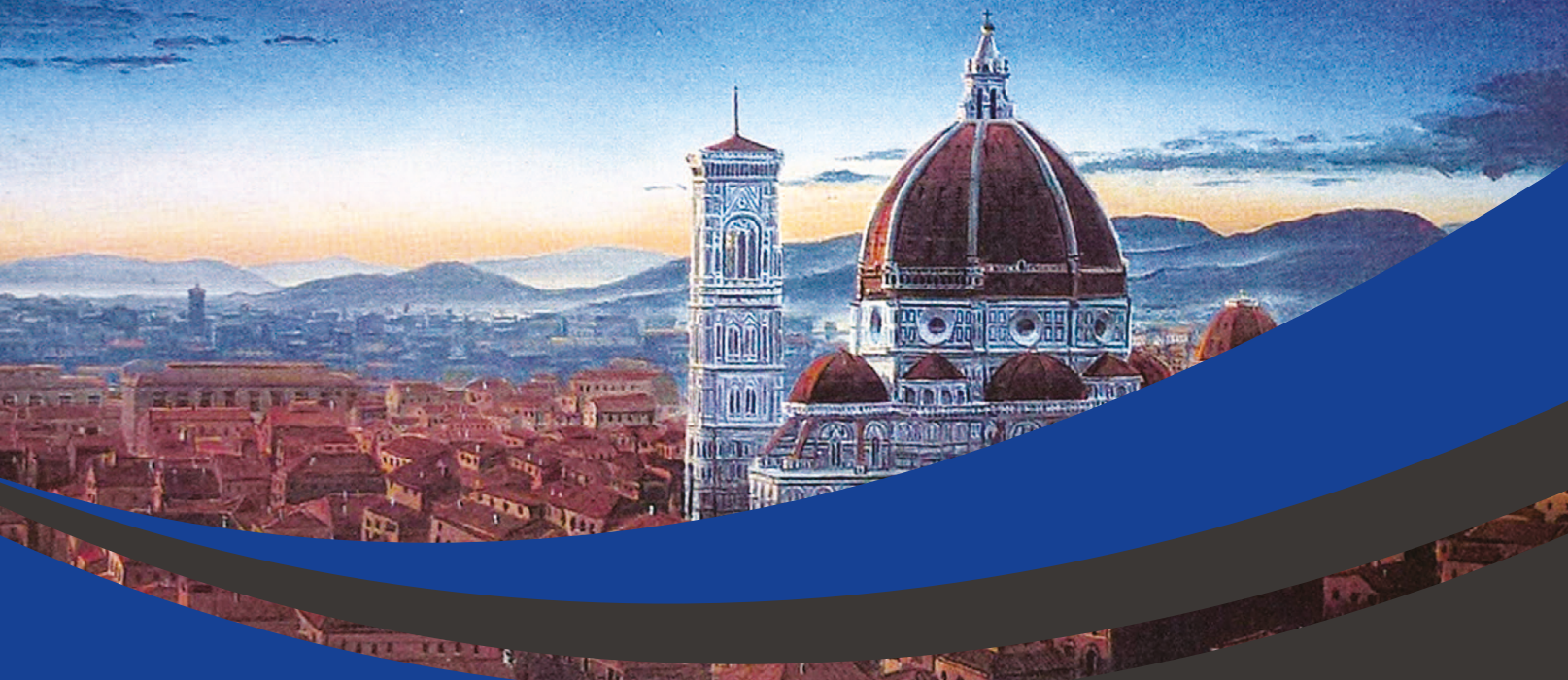

Firenze, 4-8 Febbraio 2019

\title{
XV Congresso Nazionale di Scienze Planetarie
}


PRESIDENTE DEL CONGRESSO:

Giovanni Pratesi.

COMITATO SCIENTIFICO:

Giovanni Pratesi, Emanuele Pace, Marco Benvenuti, John Brucato, Fabrizio Capaccioni, Sandro Conticelli, Aldo Dell'Oro, Carlo Alberto Garzonio, Daniele Gardiol, Monica Lazzarin, Alessandro Marconi, Lucia Marinangeli, Giuseppina Micela, Ettore Perozzi, Alessandro Rossi, Alessandra Rotundi.

COMITATO ORGANIZZATORE:

Cristian Carli, Mario Di Martino, Livia Giacomini, Vanni Moggi Cecchi, Emanuele Pace, Giovanni Pratesi, Giovanni Valsecchi.

CURATORI DEL VOLUME

Giovanni Pratesi, Fabrizio Capaccioni, Emanuele Pace, John Brucato, Marco Benvenuti, Sandro Conticelli, Aldo Dell'Oro, Daniele Gardiol, Carlo Alberto Garzonio, Monica Lazzarin, Alessandro Marconi, Lucia Marinangeli, Giuseppina Micela, Ettore Perozzi, Alessandro Rossi, Alessandra Rotundi.

Papers, data, figures, maps and any other material published are covered by the copyright own by the Società Geologica Italiana.

DISCLAIMER: The Società Geologica Italiana, the Editors are not responsible for the ideas, opinions, and contents of the papers published; the authors of each paper are responsible for the ideas opinions and contents published.

La Società Geologica Italiana, $i$ curatori scientifici non sono responsabili delle opinioni espresse e delle affermazioni pubblicate negli articoli: l'autore/i èlsono illi solo/i responsabile/i. 


\begin{abstract}
INDEX
EDUCARE ALLA SCIENZA, COMUNICARE LA SCIENZA. Giornata di formazione

Capaccioni F. - L’esplorazione del Sistema Solare: sfide tecnologiche e scoperte scientifiche

Brucato J. - Vita extraterrestre

Pace E. - L'Esplorazione planetaria oltre il Sistema Solare, a caccia di simil-terre

Gardiol D. - Tutti a caccia di bolidi e meteoriti con PRISMA. (Prima Rete Italiana per la Sorveglianza sistematica di Meteore e Atmosfera)

Maraviglia A. - Comunicare le scienze, strumenti e attività. La testimonianza da Unifi

Giacomini L., Galliani M., Malaspina M. \& Sandrelli S. - Didattica e Divulgazione in INAF e il progetto didattico "Pianeti in una stanza".
\end{abstract}

\title{
PLANETARY SYSTEMS.
}

Alei E. \& Claudi R. - Matched Exoplanet Catalog with VO connection

Alei E., Petralia A., Locci D., Claudi R., Cecchi Pestellini C., Ciaravella A. \& Micela G. - MAGRATHEA: 1D radiative-convective code for terrestrial atmospheres

Benatti S., Damasso M. \& Desidera S. - A test case to understand planetary migration mechanisms from system architecture

Bignamini A., Benatti S., Claudi R. \& Molinaro M. - GAPS Time Series resource and service implementation

Capria M.T. - Exocomets and comets .

Claudi R., Benatti S. \& Desidera S. - GAPS2.0: Hunting young planets

Fedele D. - ALMA highlights on protoplanetary disks.

Garrido A., Maldonado J., Affer L., Micela G., Petralia A., Maggio A. \& HADES collaboration - M stars. Activity Vs Planets

Giacobbe P., Bonomo A. \& Nascimbeni V. - GAPS2.0: a GIANO view of the atmosphere of hot Jupiters......

Ivanovski S.L., Turrini D., Capria M.T., Polychroni D., Zakharov V.V., Della Corte V. \& Rotundi A. - Nonspherical dust dynamics in protoplanetary disks: how dust particle realistic shapes change the dust evolution timescales

Lazzoni C., Desidera S. \& Marzari F. - Dynamical Models to Explain Double Debris Belts Systems and Comparison with SPHERE Observations.

Molinaro M., Alei E., Benatti S., Bignamini A., Bonnarel F., Damasso M., Louys M., Maris M. \& Nascimbeni V. - A Data Model for discovery and description of Exoplanetary Systems Datasets.....

Pace E. - La missione spaziale ARIEL

Pinto L.D., Magni G., Capaccioni F. \& Capuzzo-Dolcetta R. - Evolution of protoplanetary disks in Open Clusters. Material mixing in the protoplanetary matter after the perturbation of passing-by stars.

Turrini D., Oliva F., Migliorini A., Politi R., Fonte S., Fedele D., Molinari S. \& Schisano E. - ARIEL and the quest to understand planetary diversity

Zinzi A., Maris M., Alei E., Murante G., Silva L., Turrini D., Verrecchia F. \& Vladilo G. - ARTECS and ExoplAn3T: toward a synergy between two exoplanetary tools

HABITABILITY AND ASTROBIOLOGY

Balbi A., Kovacevic A.B., Tombesi F. \& Wislocka A.M. - Supermassive black holes and planetary habitability

Balucani N., Skouteris D., Caracciolo A., Casavecchia P. \& Rosi M. - Nitrogen incorporation into aromatic rings in extraterrestrial environments: toward the formation of pyridine and nucleobases 
Battistuzzi M., Cocola L., Claudi R., Alei E., Poletto L., Morosinotto T. \& La Rocca N. - An innovative setup to investigate by remote sensing growth and photosynthetic performances of cyanobacteria exposed to exo-earths simulated environments

Billi D. \& Balbi A. - Desert cyanobacteria under planetary simulations: towards future astrobiology experiments in space

Corazzi M.A., Brucato J.R. \& Poggiali G. - Photoprocessing of formamide ice: route towards prebiotic chemistry in space

Faggi S., Mumma M.J., Villanueva G.L., Paganini L. \& Lippi M. - The Volatile Composition of Comets as revealed by near-IR High-Resolution Spectroscopy

Galuzzo D., Berrilli F., Cagnazzo C. \& Giovannelli L. - 3D Simulations of Exoplanetary Atmosphere......

Longo S. \& Micca Longo G. - Is primordial atmosphere a key to organic matter delivery to early Earth? ......

Marconi A. on behalf of the HIRES Consortium - Exoplanet atmospheres with HIRES, an optical-NIR high resolution spectrograph for the ELT

Modica P., Martins Z., Meinert C. \& d'Hendecourt L. - Amino acids from photo- and thermo-processing of extraterrestrial ices: a possible source for further prebiotic chemistry.....

Mura A., Benatti S., Locci D., Micela G., Milillo A., Petralia A. \& Vladilo G. - Exoplanetary Exosphere and Exospheric tail

Murante G., Provenzale A., Vladilo G., Silva L., Maris M., Palazzi E. \& Taffoni G. - Bistability in a climate model for rocky planets

Musetti B. \& Margheritis D.B. - ExoMars RSP Mission and the developed technologies for Planetary Protection.

Provenzale A., Lenzi S. \& Murante G. - Habitability of desert-like worlds: role of albedo, evapotranspiration and carbon feedbacks.

Vladilo G., Silva L., Maris M., Murante G., Provenzale A., von Hardenberg J., Palazzi E., Simonetti P., Fulle M. \& Ivanovski S.L. - On the challenge of quantifying the habitability of extrasolar planets .

\section{PLANETS AND SATELLITES}

Adriani A., Mura A., Bolton S. \& the Juno team - The Jupiter's Atmosphere from Juno .

Altieri F., Moriconi M.L., Dinelli B.M., Mura A., Adriani A., Migliorini A., Plainaki C., Grassi D., Tosi F., Sindoni G., Piccioni G., Noschese R., Sordini R., Cicchetti A. \& the JIRAM team - Temporal and Morphological Variability of Auroral Emissions from Juno/JIRAM data.

Baioni D. - Karst landforms within Cagli crater, Mars as useful lithological and paleo-climatic markers........

Bellucci G., Vandaele A.C., Lopez-Moreno J.-J., Patel M.R., Daerden F., Depiesse C., Mason J., Ristic B., Thomas I.R., Willame Y., Oliva F., D’Aversa E., Altieri F. \& Carrozzo F.G. - The NOMAD experiment on board TGO/Exomars 2016: First results.

Bertoli S., Pacifici A., Baroni C. \& Salvatore M.C. - Geomorphological mapping of Cilaos - Gasa impact crater complex .....

Brunetti M.T., Ardizzone F., Peruccacci S. \& Guzzetti F. - Geometrical properties of landslides on Earth and Mars.

Camplone V., Sindoni G., Negri B., Ammannito. E., Altieri. F., Carrozzo F.G., Cremonese G., Pajola M., Pirrotta S. \& Zinzi A. - Identification and characterization of possible Martian landing sites.

Carli C., Serventi G., Giacomini L., Pozzobon R. \& Sgavetti M. - Proclus Crater: mapping the spectral variability within Lunar Highlands.

Cremonese G., Capaccioni F., Capria M.T., Palumbo P., Debei S., Bettanini C., Cicchetti A., Da Deppo V., De Sanctis M.C., Filacchione G., Massironi M., Noschese R., Politi R., Re C., Simioni E., Slemer A. \& Zusi M. - SIMBIO-SYS present and future.

De Marchi F., Di Achille G., Di Benedetto M., Di Stefano I. \& Cappuccio P. - Investigation of superficial features of the Galilean moons by 3GM experiment aboard ESA JUICE mission

Di Achille G. \& Vaz D.A. - Martian fan-deltas and the $\Delta \mathrm{H} 2 \mathrm{O}$ throughout the geological history of Mars: insights from sedimentary mass balance..... 
Di Achille G., Salese F. \& Vaz D.A. - Geomorphology and Sedimentology of southwestern Lake General Carrera/Buenos Aires (Chile/Argentina): Implications for Martian fluvio-lacustrine deposits.

Di Benedetto M., Cappuccio P., Ciarcia S., Iess L., Kaspi Y. \& Mann R. - The 3GM experiment on board the ESA JUICE mission.

Di Stefano I., Cappuccio P., De Marchi F. \& D’Achille G. - Determination of the Mercury's Moho depth with ESA BepiColombo MORE.

Durante D., Notaro V., Racioppa P. \& Iess L. - A peek inside the gas giants with gravity measurements.........

Fiorucci S., Podda S., Collu C., Brunetti M.T., Melis M.T. \& Scaioni M. - Detection, classification and mapping of rilles in the northern hemisphere of the Moon .

Galluzzi V., Giacomini L., Guzzetta L., Ferranti L., Massironi M. \& Palumbo P. - Regional-scale study of the structural asset of the high-Mg region of Mercury.

Genova A. - Mercury's Crust and Core from MESSENGER Geodetic Investigation......

Giacomini L., Aloisi F., De Angelis I. \& Capretti S. - Planets in a Room: a DIY, low-cost educational kit to teach Planetology.

Giacomini L., De Sanctis M.C., Ivanovski S.L., Capria M.T., Gatti L., Galluzzi V. \& Lucchetti A. - The new Italian Hub of Europlanet Society

Giacomini L., Galluzzi V., Carli C., Zambon F., Massironi M., Ferranti L. \& Palumbo P. - Geological mapping of the Kuiper (H06) quadrangle of Mercury

Guzzetta L., Giacomini L., Ferranti L. \& Palumbo P. - Structural analysis of the north-eastern sector of the Caloris basin

Ivanovski S.L., Milillo A., Kartalev M., Massetti S., Orsini S., Sordini R., Mura A., Mangano V., De Angelis E., Rispoli R., Lazzarotto F., Aronica A. \& Kazakov A. - Modelled magnetic reconnection at the Mercury's magnetopause in support of the scientific objectives of SERENA.

Lucchetti A., Pajola M., Semenzato A., Galluzzi V., Munaretto G., Cremonese G., Massironi M. \& Palumbo P. - Global properties of hollows on the surface of Mercury.

Luzzi E., Rossi A.P., Carli C. \& Altieri F. - Surface geology of Arsinoes Chaos: morphological and spectral affinities across Chaotic terrains on Mars.

Maccone C. - Moon Farside Radiotelescope and its Protection.

Marinangeli L., Tangari A.C., Pompilio L., Scarciglia F. \& Piluso E. - Clay formation in Margaritifer Chaos, Mars....

Milillo A., Mangano V., Massetti S., Mura A., De Angelis E., Ivanovski S.L., Orsini S., Rispoli R., Lazzarotto F., Aronica A. \& Kazakov A. - Exospheric Na distributions along the Mercury orbit with the THEMIS telescope .

Moriconi M.L., Adriani A., Grassi D., Mura A., Altieri F. \& the JIRAM team - Power spectral analysis of Jupiter's polar clouds by JIRAM data

Mura A., Adriani A. \& Bolton S. - Jupiter's Magnetosphere from Juno

Oliva F., Bellucci G., D’Aversa E., Altieri G., Carrozzo F.G., Daerden F., Depiesse C., Lopez-Moreno J.-J., Mason J., Patel M.R., Ristic B., Thomas I.R., Vandaele A.C. \& Willame Y. - Mars dust characterization by means of TGO/NOMAD data analysis.

Orosei R., Lauro S. E., Pettinelli E., Cicchetti A., Coradini M., Cosciotti B., Di Paolo F., Flamini E., Mattei E., Pajola M., Soldovieri F. \& Cartacci M. - Radar evidence of subglacial liquid water on Mars

Pacifici A., Ori G.G., Aboudan A., Murana A., Di Pietro I. \& Lorenzoni L - ExoMars 2020 Oxia Planum landing area characterization and performance assessment for Surface Platform landing, Rover egress and traversability

Pajola M., Pozzobon R., Simioni E., Teodoro L., Wilson J., Kling A., Salese F., Lucchetti A., Munaretto G.,8, Massironi M., Cremonese G., Sauro F., Silvestro S., Marinangeli L., Pompilio L., Tangari A.C., Noe Dobrea E. \& De Toffoli B. - Mars Human Exploration: the Vernal crater landing site

Pantaloni M., Graciotti R., Roma M., Carta R., D’Orefice M., Campo V., Pondrelli M., Marinangeli L., Massironi M., Pozzobon R., Ferrari S., Carli C., Galluzzi V., Ferranti L., Mancinelli P., Pauselli C., Melis M.T., Rossi A.P. \& Zinzi A. - Planetary geological mapping: integrating Earth-based protocols with the support of the Italian Geological Survey. 
Provenzale A. \& Lenzi S. - Toward a simulation of ice-covered oceans.

Pompilio L. \& Marinangeli L. - The intriguing case of Ladon Vallis, Mars

Re C., Zinzi A., Cremonese G., Simioni E., Mudric T. \& Capria M.T. - The CaSSIS data management and visualization on ASI-SSDC

Rognini E., Capria M.T. \& Zinzi A. - Mercury surface temperature distribution

Silvestro S., Chojnacki M., Vaz D.A., Cardinale M., Mongelluzzo G. \& Esposito F. - First evidence for brighttoned megaripple migration on Mars

Tosi F., Mura A., Adriani A., Filacchione G., Sindoni G., Altieri F., Grassi D., Migliorini A., Moriconi M.L., Piccioni G., Plainaki C., Turrini D., Cicchetti A., Noschese R. \& Sordini R. - Thermal mapping of Io obtained with the JIRAM instrument onboard the Juno mission .....

Zambon F., Carli C., van der Bogert C.H., Hiesinger H., Altieri F., Massironi M. \& Capaccioni F. - Spectral analysis of the Apollo basin on the Moon

Zambon F., Carli C., Wright J., Altieri F., Giacomini L., Massironi M., Capaccioni F., Rothery D.A., Cremonese G. \& the PLANMAP Team - Spectral variations across H-05 Hokusai quadrangle on Mercury: a connection between spectral properties and morpho-stratigraphic units

Zinzi A., Giardino M., Sindoni G., Ammannito E., Perozzi E., Plainaki C., Capria M.T. \& Palomba E. MATISSE 2.0: towards a hub for the planetary science community.

DYNAMICS OF CELESTIAL BODIES, EITHER NATURAL OR MAN-MADE

Buzzoni A., Guichard J., Matassoni F., Figer A., Altavilla G. \& Galleti S. - Toward a physical characterization of the Soviet/Russian constellation of Molniya satellites

Cibin L., Chiarini M., Bernardi F., Ragazzoni R., Salinari P., Sessler G. \& Kugel U. - The Fly Eye Telescope is growing up - Development status and first lights from Sky....

Di Cecco A., Perozzi E., Guarcello M., Iacovone D., Bianco G., Buzzoni A., Di Paola A., Falvella M.C., Fierro D., Marzo C. \& Micela G. - Astronomical seeing evaluation for NEO surveys at Monte Mufara and at the ASI-CGS in Matera.

Di Cintio P., Rossi A. \& Valsecchi G.B. - Numerical simulations of space debris populations with symplectic integrators

Dotto E., Mazzotta Epifani E., Perna D., Ieva S., Perozzi E., Lazzarin M. \& Bertini I. - Physical Properties of Near Earth Objects: the legacy of the EU H2020 programme NEOShield-2

Lari G., Saillenfest M. \& Fenucci M. - Orbital evolution of the Galilean satellites driven by tidal dissipation

Lucchesi D.M, Anselmo L., Bassan M., Magnafico C., Pardini C., Peron R., Pucacco G. \& Visco M. - State of the art of the measurement of the Lense-Thirring effect after a century from its formulation

Mazzotta Epifani E. on behalf of the LICIACube team - LICIACube: the Light Italian Cubesat for Imaging of Asteroid in support to the NASA DART mission.

Nastasi A., Spanò P., Masiero S. \& Ziino L. - The new wide-field instruments installed on Madonie Regional Natural Park to study and monitor fast moving and transient phenomena.

Perna D. - The "Near-Earth Space Trekker" (NEST) multi-asteroid mission concept.

Santoli F., Iafolla V., Fiorenza E., Lefevre C., Lucchesi D.M., Lucente M., Magnafico C. \& Peron R. - The BepiColombo ISA accelerometer: flying to Mercury

Serra D., Lari G., Tommei G. \& Milani A. - A long tradition of orbit determination in Pisa: the Juno mission to Jupiter

Tommei G. - Impact Monitoring of Near-earth Objects: past, present and future challenges

Valsecchi G.B. - Comparing NEA surveys

Ciarniello M., De Sanctis M.C., Ammannito E., Raponi A., Carrozzo F.G., Longobardo A., Tosi F., Rognini E., Zambon F., Schröder S., Raymond C.A., Russell C.T. \& the VIR team - Opposition effect on Ceres from VIR-Dawn observations. 
Ciarniello M., Capaccioni F., Filacchione G., De Sanctis M.C., Capria M.T., Raponi A., Formisano M., Tosi F., Longobardo A., Rinaldi G., Erard S., Bockelee-Morvan D., Leyrat C., Arnold G., Barucci M.A., Quirico E., Fornasier S., Kappel D., Rousseau B., Mottola S. \& the Rosetta/VIRTIS Team - The seasonal cycle of water ice at the surface of 67P/Churyumov-Gerasimenko as observed by VIRTIS-Rosetta..............

Dell'Oro A. - Monte Carlo investigation of asteroid collisions statistics

Formisano M., Federico C., De Sanctis M.C. \& Magni G. - Numerical simulations of thermal convection: applications to the dwarf planet Ceres.

Ieva S., Dotto E., Mazzotta Epifani E., Perna D., Micheli M., Brucato J.R., Poggiali G., Antoniucci S., Valsecchi G.B. \& Perozzi E. - Carbonaceous near-Earth objects: a survey of the primitive material in the Solar System

Lazzarin M., Petropoulou V., Bertini I., La Forgia F. \& Migliorini A. - The surface variability of 3200 Phaethon: implication for its origin and evolution

Longobardo A., Della Corte V., Rotundi A., Rinaldi G., Zakharov V.V., Ivanovski S.L., Formisano M., Palomba E., Dirri F. \& Palumbo P. - 67P/Churyumov-Gerasimenko's dust activity from pre- to post- perihelion as detected by Rosetta/GIADA.

Lucchetti A., Penasa L., Pajola M., Massironi M., Brunetti M.T., Cremonese G. \& OSIRIS-REx Team Landslides on Comet 67P/Churyumov-Gerasimenko Yarkowski evolution of asteroid families

Migliorini A., De Sanctis M.C., Lazzaro D., Lazzarin M., La Forgia F., Barbieri M. \& Mesa D - Insights of the Vesta family and non-family members spectroscopic properties

Pajola M., Della Giustina D., Bennett C., Burke K., Lauretta D.S., Rizk B., Delbo M., Walsh K., Brucato J.R., Dotto E., Bierhaus E.B., Campins H., Daly M., Elder C., Michel P., Molaro J., Nolan M.C., Schwartz S.R. \& The OSIRIS-REx Team - Scientific Analysis of the Size-Frequency Distribution of Boulders $\geq$ $10 \mathrm{~m}$ on Asteroid (101955) Bennu

Paolicchi P., Spoto F., Knezevic Z. \& Dell'Oro A. - The V-plot of asteroid families, and the relevance of the initial conditions: a preliminary analysis.

Raponi A., Ciarniello M., Filacchione G., Capaccioni F., De Sanctis M.C., Moroz L.V., Vinogradoff V., Tosi F., Arnold G., Quirico E., Mennella V., Beck P., Pilorget C., Pommerol A., Schröder S., Kappel D., Istiqomah I., Rousseau B. \& Poch O. - Aliphatic organics on comet 67P: from interstellar dust to pristine solar system material

Rinaldi G., Formisano M., Kappel D., Capaccioni F., Bockelée-Morvan D., Cheng Y.-C., Vincent J-B., Deshapriya P., Arnold G., Capria M.T., Ciarniello M., D’Aversa E., De Sanctis M.C., Doose L., Erard S., Federico C., Filacchione G., Fink U., Leyrat C., Longobardo A., Magni G. el al. - Analysis of a night side dust activity on comet 67P observed by VIRTIS-M: A new method to constrain the surface thermal inertia.....

Rognini E., Capria M.T., Tosi F., De Sanctis M.C., Ciarniello M., Longobardo A., Carrozzo F.G., Raponi A., Frigeri A., Palomba E., Fonte S., Giardino M., Ammannito E., Raymond C.A. \& Russell C.T. - Ceres' thermal inertia from Dawn data

Zakharov V.V., Ivanovski S.L., Della Corte V. \& Rotundi A. - Analytical models for the dusty-gas near nucleus coma.......

\section{METEORS AND METEORITES, IDPS \& SAMPLES RETURNED FROM MINOR BODIES.}

Barbaro A., Domeneghetti M.C., Meneghetti M., Litti L., Fioretti A.M., Goodrich C., Shaddad M.H., Alvaro M., \& Nestola F. - Graphite-based geothermometry of Almahata Sitta ureilites

Barghini D., Gardiol D. \& Carbognani A. - Assessment of astrometric performances for PRISMA all-sky cameras

Bellesi M., Giuli G. \& Santini C. - Classification of two meteorites from North-Western Africa (NWA), characterization of the trace elements found in phosphide inclusions and analysis of the crystalline nano-phases contained in the fusion crusts and/or the shock veins

Bonadiman C., Lugari C., Pelorosso B., Lanzafame G., Mancini L., Vaccaro C. - Chemical and physical characteristics of a new chondrite from Draa Valley, Tata (Morocco) 
Carbognani A. - MuFiS - Multipurpose Fireball Software.

Carli C., Pratesi G., Moggi-Cecchi V. - Effects of mineralogical and petrographic variability on the VIS-NIR spectral features of HED meteorites

De Angelis S., Carli C., Ferrari M., Manzari P., Vinogradoff V., De Sanctis M.C., Pratesi G. \& Moggi Cecchi V. - VNIR spectroscopy of Olivine grains in Achondrites Meteorites ....

Dionnet Z., Longobardo A., Rotundi A., Suttle M.D., Brunetto R., Folco L., Gemelli M., Della Corte V. \& King A. - Non destructive pre-characterization of micrometeorites by $\mathrm{X}-\mu \mathrm{CT}$.

Gardiol D. \& the PRISMA collaboration. - PRISMA italian fireball network: status update

Hu J., Asimow P.D., Ma C. \& Bindi L. - New compositions of shock-synthesized AlCuFe icosahedral quasicrystals: implications for the impact origin of natural quasicrystals in the Khatyrka meteorite.....

Longobardo A., Dionnet Z., Rotundi A., Suttle M.D., Brunetto R., Folco L., Gemelli M. \& Della Corte V. Infrared spectroscopy of micrometeorites' interior and exterior

Lugari C., Bonadiman C., Cruciani G., Franceschelli M., Marchi M. \& Tassinari R. - New data and revision of classfication of the Sinnai meteorite (Cagliari)

Mancuso S., Taricco C., Colombetti P., Rubinetti S., Sinha N., Bhandari N., Barghini D. \& Gardiol D. - 44Ti activity in meteorites and assessment of the long-term heliomagnetic field variation

Marinucci W., Arzilli F., Mancini L., Cicconi M.R., Folco L., Glass B.P., Koeberl C., Pratesi G. \& Giuli G. Bubble size distribution of microtektites from the Australasian strewn field.

Moggi Cecchi V., Pratesi G., Ghiara M.R., Rossi M., Gardiol D. \& Ascione G. - The Sannio meteorite: a new chondrite from Southern Italy.

Nava J., Suttle M.D., Carli C., Spiess R., Folco L., Najorka J., Palomba E. \& Massironi M. - MM 5.29: a unique $\mathrm{CV}$-like micrometeorite as evidence of hydrothermal activity on $\mathrm{CC}$ parent bodies

Pittarello L., Goderis S., Soens B., McKibbin S.J., Bariselli F., Barros Dias B.R., Helber B., Giuli G., Lepore G.O., Yamaguchi A., Roszjar J., Debaille V., Koeberl C., Magin T. \& Claeys P. - Fusion crust and atmospheric entry of ordinary chondrites, a comparison between experiments and nature

EXPERIMENTAL AND LABORATORIAL PLANETOLOGY.

Brucato J.R., Meneghin A. \& the EURO-CARES Team - EURO-CARES: Conceptual design and procedures for a European sample curation facility for sample return

Coccaro F., Filippone R., De Angelis G. \& Di Iorio A. - A luminescence In-Situ instrument for planetary dating applications.

Fastelli M., Comodi P., Zucchini A., Maturilli A., Balic-Zunic T. \& Rossi M. - Reflectance and emissivity measurements on hydrated salts: a tool to dive into icy planets.

Ferrari M., De Angelis S., De Sanctis M.C., Altieri F. \& Ammannito E. - Laboratory activities in support to the Ma_MISS experiment onboard the ExoMars2020 rover .

Ferri A., Cassi C., Massobrio F., Centuori S. \& Pellacani A. - The European contribution to Mars Sample Return mission as key opportunity for planetary science.

Fulvio D., Ieva S., Perna D., Mazzotta Epifani E., Strazzulla G. \& Dotto E. - Space weathering effects on V-type asteroids

Gabbai F., Lauro S.E., Cosciotti B., Di Paolo F., Mattei E., Pettinelli E., Pratesi G. \& Carli C. - Electrical chondrite characterization for the radar propagation study in planetary crusts.

Mancarella F., D’Elia M., Orofino V., Micca Longo G. \& Longo S. - Laboratory measurements on decomposition of White Soft Minerals (WSM) under conditions of interest to planetology

Masiero S., Nastasi A. \& Ziino L. - GAL Hassin, the new center for Outreach of Astronomy and Physical Sciences in Sicily.

Migliorini A., Ioppolo S., Kanuchova Z., Strazzulla G., Piccioni G. \& Tosi F. - Molecular oxygen in the surface of Galilean moons investigated in laboratory.....

Murri M., Cámara F., Adam J., Domeneghetti M.C. \& Alvaro M. - Intracrystalline geothermometry applied to a Martian analogue 
Pisello A., De Sanctis M.C., Maturilli A., De Angelis S., Ferrari M., Vetere F., Pauselli C. \& Perugini D. Experimental petrology and planetary studies: spectroscopy on silicate glasses for the exploration of volcanic terrains

Poggiali G., Brucato J.R., Corazzi M.A., Meneghin A., Ieva S., Dotto E., Mazzotta Epifani E. \& Fornaro T. Laboratory study in support of OSIRIS-REx mission. Mineral and organics reflectance spectra in space simulated environment as hint for a deep investigation of Bennu surface.

Stefani S., Piccioni G. \& Snels M. - Experimental set up used to characterize the optical properties of gases at typical planetary conditions

Strazzulla G. - Ion and electron bombardment of materials studied in the laboratory in support of planetary space missions

Strumia F., Brez A., Bacci P. \& Paolicchi P. - The use of a quantum filter in astronomical imaging 


\section{EDUCARE ALLA SCIENZA, COMUNICARE LA SCIENZA}

Giornata di formazione 


\title{
L'esplorazione del Sistema Solare: sfide tecnologiche e scoperte scientifiche
}

\author{
Capaccioni F.
}

Direttore dell’Istituto di Astrofisica e Planetologia Spaziali (INAF)

Corresponding author email: fabrizio.capaccioni@iaps.inaf.it

L'esplorazione del Sistema Solare per mezzo di missioni umane e di sonde robotiche ha rappresentato negli ultimi 60 anni una delle grandi avventure nella storia dell'Umanità ed ha permesso di rivoluzionare completamente la nostra comprensione della miriade di corpi orbitanti attorno al Sole. Missione dopo missione, nuove ed emozionanti scoperte hanno contribuito a modificare la nostra visione del Sistema Solare. Dai depositi di ghiaccio esistenti nei poli lunari, alle piogge di Metano liquido che, sulla superficie di Titano, generano fiumi e laghi. Dalla scoperta di un clima marziano caldo ed umido che può aver determinato condizioni ambientali favorevoli alla nascita della vita, alla scoperta di vulcani potenzialmente attivi sulla superficie di Venere. Dalle bizzarre strutture geometriche determinate dalla circolazione atmosferica nelle regioni polari di Saturno, alla scoperta di processi dinamici attivi negli anelli di Saturno che possono aiutare la comprensione dei meccanismi di accrescimento planetario. Dallo studio delle comete ed asteroidi primitivi che ci hanno fornito indicazioni sulle prime fasi di formazione del Sistema Solare, alla potenziale presenza di oceani di acqua liquida nel sottosuolo di Europa, Titano ed Encelado.

Questi ed altri sensazionali risultati scientifici hanno richiesto sviluppi tecnologici altrettanto sensazionali per mettere in grado gli scienziati di realizzare dei progetti a volte visionari. Le nuove tecnologie sviluppate nel corso di questi progetti hanno trovato proficue applicazioni nella nostra vita quotidiana. Pensiamo ad esempio alle schiume a memoria di forma (Memory Foam) che, studiate inizialmente per proteggere gli astronauti sono ora usate correntemente per la produzione di materassi e di caschi protettivi, o alle tele termiche, teli leggerissimi e riflettenti ma estremamente efficaci come materiale isolante in situazioni di emergenza.

Le missioni spaziali di esplorazione hanno permesso di investigare tutti i pianeti del sistema solare, da Mercurio il più vicino al Sole fino a Plutone ed oltre: di circa un mese fa l'incontro ravvicinato della sonda New Horizon con un corpo della fascia di Kuiper (2014MU69 denominato Ultima Thule) alla distanza di 45 Unità Astronomiche (circa 7 miliardi di km dal Sole). Le sonde Voyager hanno superato i limiti del Sistema Solare e rappresentano perciò i nostri messaggeri nello spazio interstellare, oltre ad essere le missioni più longeve (sono state infatti ideate negli anni ' 70 ) in operazione.

Le sonde spaziali, equipaggiate con sofisticate strumentazioni, attraverso lo studio dei singoli corpi del Sistema Solare permettono di individuare degli ambiti di ricerca che sono fondamentali per la comprensione del ruolo dell’Uomo nell'Universo:

- Lo studio dei processi fisici e chimici che agiscono all'interno, sulla superficie e nelle atmosfere (ove presenti) dei pianeti e satelliti, rappresenta una fondamentale opportunità per ricavare informazioni sul nostro pianeta, la Terra, sulla sua storia e sulla sua evoluzione.

- I corpi primitivi, comete ed asteroidi, pressoché inalterati dal momento della loro formazione ci permettono decifrare le tracce residue dei processi attivi nelle fasi primordiali e ricavare informazioni sull'origine del Sistema Solare.

- Quali sono i processi che rendono un ambiente abitabile? quali sono le sorgenti di materiale organico nel Sistema Solare? Marte può aver sperimentato nell'arco della sua storia condizioni adatte all'attività biologica? Gli interni dei satelliti dei pianeti giganti hanno oggi condizioni adatte all'attività biologica?

L'Italia riveste un ruolo molto importante nell'esplorazione del Sistema Solare attraverso i suoi ricercatori e le sue industrie coordinati dall'Agenzia Spaziale Italiana sia in ambito continentale, nell'Agenzia Spaziale Europea (ESA), che grazie ai rapporti di collaborazione bilaterale con le maggiori agenzie spaziali internazionali a cominciare dalla NASA. 


\section{Vita extraterrestre}

Brucato J.

Membro dello Science Team di OSIRIS-Rex - Osservatorio Astrofisico di Arcetri (INAF)

Corresponding author email: john.brucato@inaf.it

Esiste vita nello spazio? Lo studio della nascita della vita sulla Terra e la ricerca di forme di vita extraterrestre sono argomenti che, oltre ad esercitare un grande fascino sul pubblico, rappresentano soprattutto un indirizzo nuovo che la scienza attuale vuole intraprendere per cercare risposte a domande che da sempre accompagnano l'uomo.

Oggi sappiamo che la presenza della vita sulla Terra è legata all'origine ed evoluzione del Sistema Solare e alle condizioni iniziali presenti nella nube molecolare interstellare dalla quale il nostro pianeta ha avuto origine. La vita, così com'è conosciuta sulla Terra, è originata da complesse reazioni basate sulla chimica del carbonio, probabile risultato dell'interazione di molecole organiche e materiale inerte come i minerali. Tuttavia, le condizioni ambientali in cui la vita si è sviluppata sulla Terra primordiale non sono ancora del tutto note, e inoltre le tracce della più antica storia del nostro pianeta sono state, purtroppo, cancellate dall'attività geologica. Ancora oggi mancano le prove definitive della composizione dell'atmosfera primordiale, della temperatura superficiale, il $\mathrm{pH}$ oceanico, e di tutte le altre condizioni ambientali generali e locali importanti per la comparsa dei primi organismi viventi sulla Terra.

Quello che sappiamo è che la vita sul nostro pianeta è apparsa subito ed ha assunto, nel corso delle ere geologiche, svariate forme ciascuna con diversi gradi di complessità. Ma siamo in grado di riconoscere le impronte che la vita può aver lasciato nel nostro Sistema Solare o in pianeti extrasolari?

Le ricerche in ambito spaziale di luoghi dove la vita può essere presente o essersi sviluppata nel passato si concentrano su quegli oggetti dove l'acqua liquida può esistere o può essere esistita in passato. La presenza di acqua liquida è fondamentale per permettere l'esistenza di reazioni chimiche complesse in quanto solvente universale in grado di formare legami ad idrogeno con i soluti polari. Nel nostro sistema solare i luoghi nei quali possiamo trovare l'acqua sono Marte, le lune ghiacciate di Giove Europa ed di Saturno Encelado, i grandi asteroidi e le comete.

Oggi l'obiettivo di questa nuova e straordinaria avventura è cercare segni di vita nel nostro Sistema Solare ma anche mondi abitabili in pianeti extrasolari con caratteristiche e collocazione planetaria che possano permettere una vita paragonabile, almeno biochimicamente, a quella terrestre. I candidati già ci sono, ma le informazioni che oggi riusciamo ad avere su di essi sono ancora troppo vaghe. La ricerca di esistenze extraterrestri, infatti, è strettamente legata al significato che si dà alla parola "vita", sul quale gli scienziati continuano ancora oggi a dibattere. L'esplorazione spaziale ha, quindi, l'obbiettivo principale di trovare e decifrare i segni che forme di vita presente o passata possono aver lasciato nello spazio. In questo intervento si parlerà delle recenti missioni spaziali, le tecniche con le quali poter trovare la vita nello spazio e quali sono le implicazioni culturali e sociali che una scoperta così rivoluzionaria porterà. 


\title{
L'Esplorazione planetaria oltre il Sistema Solare, a caccia di simil-terre
}

\author{
Pace E. \\ National Program Manager della missione ARIEL - Dipartimento di Fisica e Astronomia, Università degli Studi di \\ Firenze \\ Corresponding author email: emanuele.pace@unifi.it, pace@arcetro.astro.it
}

L'illuminata intuizione di Giordano Bruno sui molti mondi, sulla non unicità della terra, è ormai una realtà dimostrata: nel 1992 il primo annuncio di un pianeta orbitante intorno ad un'altra stella. Trattandosi di una stella molto speciale, una pulsar, non si credette possibile. Si attese il 1995 per l'annuncio da parte di Mayor e Queloz dell'Osservatorio di Ginevra della scoperta di un pianeta di dimensioni paragonabili a quelle di Giove (51 Pegasi b) orbitante la stella 51 Pegasi. Da oltre vent'anni quindi, gli astronomi utilizzano strumenti sempre più sofisticati per studiare il cosmo alla ricerca di pianeti orbitanti intorno alle stelle che ogni notte illuminano il nostro cielo. Telescopi spaziali e grandi telescopi a terra scrutano ogni stella alla ricerca di segnali che permettano di individuare la presenza di uno o più pianeti. Non riusciamo, se non in pochissimi casi, a osservare direttamente il pianeta, siamo costretti piuttosto a evidenziare fenomeni che diano informazioni indirette della presenza dei pianeti. Tra i metodi più utilizzati ci sono il metodo dei transiti e il metodo delle velocità radiali: il primo sfrutta la riduzione del flusso di luce che arriva a noi dovuto al passaggio del pianeta davanti alla stella (di fatto un'eclisse), il secondo invece osserva il movimento della stella, dovuto all'attrazione che il pianeta esercita sulla stella, attraverso lo spostamento delle righe spettrali legato all'effetto Doppler. Il motivo di tanta attenzione è radicato nell'antica domanda riguardante l'unicità della Terra come pianeta vivente e soprattutto sulla possibilità che la vita si sia formata ed evoluta altrove.

Altro tema di grande interesse è la formazione e l'evoluzione dei sistemi planetari. Finora conoscevamo solo il Sistema Solare, un sistema di pianeti strutturato in pianeti interni rocciosi e pianeti esterni giganti gassosi o ghiacciati. In base ad esso, i ricercatori avevano formulato dei modelli di formazione ed evoluzione planetaria che si sono rivelati non applicabili alla generalità dei sistemi che si andavano via via scoprendo. Grazie al gran numero di pianeti e sistemi planetari scoperti intorno a stelle di dimensioni molto diverse tra loro, è possibile avere una popolazione statistica che permetterà agli astronomi di definire meglio i modelli.

La conferenza descriverà gli sviluppi di questa ricerca a partire dalle prime fasi, in un avvincente progresso che ci ha portato a trovare circa 4000 pianeti, a studiarne le caratteristiche e a preparare gli strumenti futuri che arriveranno addirittura a studiarne le atmosfere e la meteorologia. Tra questi strumenti, PLATO, CHEOPS e TESS sono telescopi spaziali che raccoglieranno l'eredità dei telescopi spaziali Corot e Kepler per osservare il cielo a caccia di nuovi pianeti, con l'obiettivo ambizioso di arrivare ad almeno un milione di pianeti noti. Questi strumenti spaziali sono affiancati da molti altri applicati ai grandi telescopi a terra. Nel futuro a breve, un ruolo importante sarà giocato dagli strumenti collocati al grande telescopio Extremely Large Telescope e dal telescopio ARIEL, che studierà per la prima volta le atmosfere e gli ambienti planetari. Di grande interesse sono le simil-terre, pianeti con caratteristiche simili alla terra e che quindi lasciano sperare in mondi abitati simili ai nostri. Oggi sono al limite delle nostre capacità di rivelarli, ma presto riusciremo a coglierne i deboli segnali che ne indicano la presenza. La speranza è di conoscere meglio come si sono formati i pianeti, come sono evoluti e di comprenderne la grande varietà, ma soprattutto è trovare i marcatori che inequivocabilmente diano indicazione di vita altrove. 


\title{
Tutti a caccia di bolidi e meteoriti con PRISMA (Prima Rete Italiana per la Sorveglianza sistematica di Meteore e Atmosfera)
}

\author{
Gardiol D.
}

Responsabile Scientifico di PRISMA - Osservatorio Astrofisico di Torino (INAF)

Corresponding author email: daniele.gardiol@inaf.it

PRISMA (Prima Rete per la Sorveglianza sistematica di Meteore e Atmosfera), creata nel 2016, consiste in una rete di camere all-sky per l'osservazione del cielo. La rete, sotto il coordinamento dell'INAF-Osservatorio Astrofisico di Torino (OATo), attualmente consiste di 33 camere operative e altre 15 in fase di installazione. Lo scopo scientifico principale è il monitoraggio di meteore brillanti (bolidi) per determinare l'orbita degli oggetti che le provocano e delimitare con un buon grado di approssimazione le aree dell'eventuale caduta di frammenti. L'osservazione sistematica del cielo, effettuata 24 ore su 24, consente inoltre di raccogliere dati utili per la meteorologia e il monitoraggio ambientale, con particolare riferimento ai livelli di inquinamento luminoso provocato da fonti artificiali. Al progetto PRISMA partecipano ricercatori dell'Istituto Nazionale di Astrofisica e delle Università, Gruppi Astrofili, Osservatori Astronomici e Meteorologici regionali e locali, Musei, Centri di divulgazione scientifica. Le Scuole sono coinvolte con un programma didattico e con laboratori di astronomia che intendono far partecipare gli studenti e i docenti alle attività di ricerca del progetto, fianco a fianco con i ricercatori.

PRISMA è una rete di camere all-sky per l'osservazione di meteore brillanti ("bolidi"), realizzata con l'obiettivo di determinare le orbite degli oggetti che le provocano e delimitare con un buon grado di approssimazione le aree dell'eventuale caduta di meteoriti che in genere è associata a questi eventi. A tutt'oggi in Italia si conoscono solamente una quarantina di eventi associati al recupero di meteoriti, un numero molto esiguo.

A regime la presenza di questo "fireball network" italiano consentirà di massimizzare le probabilità di recupero di meteoriti "fresche". Le meteoriti sono la principale fonte di informazioni sulla storia remota del Sistema Solare, sui meccanismi di formazione e sul materiale da cui si formarono il Sole ed i pianeti. L'osservazione sistematica di questi eventi permette inoltre di determinare il flusso degli oggetti cosmici di più piccole dimensioni che interagiscono con la Terra, parametro questo non ancora ben conosciuto. Inoltre $\mathrm{i}$ dati raccolti in maniera sistematica possono contribuire a perfezionare i modelli relativi all'interazione di corpi cosmici con l'atmosfera che a tutt'oggi presentano ancora molte lacune a causa della mancanza di un buon set di dati osservativi.

Oltre all'aspetto astrofisico, le immagini del cielo raccolte in continuo permettono il monitoraggio sistematico della copertura nuvolosa e dell'attività elettrica atmosferica. L'obiettivo finale del progetto è quello di creare una rete di stazioni osservative, con maglie che non superino i $100 \mathrm{~km}$, che si estenda su tutta l'Italia. La rete PRISMA è interconnessa ad analoghi programmi già in funzione o in corso di realizzazione in Francia (FRIPON), Olanda, Belgio e Germania. Un aspetto caratteristico del progetto è che vede coinvolti, oltre a personale scientifico dell'INAF e di varie Università, anche gruppi di astrofili, osservatori regionali e locali, planetari, docenti e studenti principalmente delle scuole superiori. Il potenziale del progetto in termini di divulgazione e coinvolgimento del pubblico anche attraverso i mass media è molto grande ed è già stato verificato. Il tema della caduta di materiale extraterrestre e la possibilità di osservare il fenomeno, comprenderne l'origine e studiarne la composizione, ha un impatto molto grande presso il pubblico.

Ad oggi sono operative 33 camere ed in fase di installazione altre 15 camere, in particolare nel Nord Italia. Ogni singola stazione della rete PRISMA è costituita da un mini-PC (NUC), da uno switch e da una camera IP con interfaccia RJ45 alimentata PoE. Dall'inizio del progetto, che fa capo all'INAF-Osservatorio Astrofisico di Torino, numerosi altri enti hanno aderito, Pubblici e Privati, Università, Associazioni, Scuole. Una intensa attività di Didattica e Divulgazione è in corso poiché il progetto ben si presta a questo tipo di attività. La rete PRISMA ha buona visibilità in vari ambiti: Stampa generalista (Repubblica, Il secolo XIX, La Stampa, Il Corriere, ...) e specializzata (Le Stelle, Coelum, ...), Radio e TV (Radio 2 nazionale "Caterpillar", RaiNews24, TGR Leonardo, TG regionali, Reteconomy, ...), oltre a numerosissimi siti web, associazioni di astrofili, eccetera. 


\title{
Comunicare le scienze, strumenti e attività. La testimonianza da Unifi
}

\author{
Maraviglia A. \\ Responsabile Unità di processo "Servizi di comunicazione", Ufficio stampa - \\ Università degli Studi di Firenze, giornalista
}

Corresponding author email: antonella.maraviglia@unifi.it

Oltre alla didattica e alla ricerca l'Università ha una terza missione: il trasferimento e la divulgazione della conoscenza. È una dimensione ormai diventata istituzionale e strutturale, nata dalla trasformazione sociale, dalla crescita tecnologica e anche dalle attese della stessa cittadinanza nei confronti dello sviluppo scientifico. E' cresciuta di conseguenza negli anni la richiesta di informazione scientifica, la domanda di divulgazione, si potrebbe dire il bisogno di esperti. In parallelo, i ricercatori universitari vengono oggi valutati - dagli organismi nazionali incaricati - anche sul public engagement, cioè su quanto i risultati della ricerca vengono comunicati, condivisi, disseminati: ai media, agli stakeholder, ai cittadini.

La testimonianza relativa all'Università di Firenze propone alcuni esempi e nello stesso tempo strumenti che possono essere utili al pubblico degli studenti più giovani e ai professionisti della comunicazione. L'iniziativa più recente è un database dal nome "Le parole della ricerca". L'Università di Firenze ha chiesto, attraverso un sondaggio on line, ai propri docenti e ricercatori di catalogare per parole chiave il proprio campo di impegno scientifico e indicare la propria disponibilità per iniziative di divulgazione e di public engagement. I dati raccolti sono incrociati con quelli relativi al ruolo, al Dipartimento di appartenenza e al settore disciplinare e consentono di avere uno strumento da poter utilizzare per la comunicazione dei risultati della ricerca e dell'offerta divulgativa dell'Università. Il database sarà fonte diretta di consultazione per gli studenti e le loro famiglie, le scuole, le aziende e più in generale i cittadini. Sarà anche una risorsa utile per i media e per $\mathrm{i}$ giornalisti.

Sempre più diffusa nella pratica della cronaca è, infatti, la ricerca dell'esperto, cioè della persona dotata di competenza garantita dal suo ruolo attivo nella ricerca, che sia in grado di spiegare e collaborare a far comprendere fatti e situazioni. Molta parte del lavoro dell'ufficio stampa di un'Università è anche quella di riuscire a mettere in contatto il giornalista con la persona giusta per il tema del giorno, per l'approfondimento o anche semplicemente per la verifica di una notizia. Far incontrare "domanda e offerta" in questo caso rappresenta, di conseguenza, una modalità di comunicazione della ricerca in atto, un trasferimento di conoscenza che risponde anche all'esigenza diffusa di nuovi divulgatori, di affabulatori scientifici, di narratori di cultura capaci di appassionare. Ma è anche un servizio per i professionisti del giornalismo: i tranelli delle fake news, la difficoltà di combattere l'eccessiva velocità dell'informazione sono elementi di un quadro complesso, nel quale il ricercatore può e deve dare un contributo di chiarezza e correttezza.

UnifiMagazine, il periodico online edito dall'Università di Firenze, in questo senso vuole rispondere a una doppia esigenza: essere strumento diretto di informazione e comunicazione - anche con un registro più disinvolto e attrattivo - e nello stesso tempo garantire, sulla base di alcuni semplici criteri di base, un'informazione scientifica corretta, verificabile, referenziata. Gli autori/ricercatori ci "mettono la faccia" e collaborano con la redazione (composta da giornalisti interni) sotto la supervisione del comitato editoriale, a spiegare quello che stanno studiando.

Per ultima ma non ultima, infine, l'esperienza di divulgazione e comunicazione scientifica di più lungo corso, quella di OpenLab, costituito dall'Università di Firenze nel 2002 con l'obiettivo di creare una struttura formativa per promuovere attività di divulgazione e diffusione della cultura scientifica.

OpenLab opera durante l'anno con iniziative aperte a tutti e con proposte rivolte alle scuole. Una manifestazione annuale della durata di una settimana, "ScienzEstate", è ormai un appuntamento fisso per tutta l'area fiorentina. Può essere utile ricordare che OpenLab è nato letteralmente sul campo, cioè nel polo scientifico dell'Università di Firenze a Sesto fiorentino, creato all'inizio degli anni Novanta, per iniziativa degli stessi ricercatori, con l'impulso dettato dall'esigenza di aprire - in primis ai cittadini del territorio -, le porte di quei laboratori, far vedere che cosa succede nel lavoro quotidiano degli scienziati. 


\title{
Didattica e Divulgazione in INAF e il progetto didattico "Pianeti in una stanza"
}

\author{
Giacomini L. ${ }^{1}$, Galliani M. ${ }^{2}$, Malaspina M. ${ }^{3} \&$ Sandrelli S. ${ }^{4}$ \\ ${ }^{1}$ Responsabile Comunicazione, Divulgazione e Didattica INAF-IAPS. \\ ${ }^{2}$ Responsabile Ufficio Stampa INAF. \\ ${ }^{3}$ Direttore Responsabile Media INAF. \\ ${ }^{4}$ Responsabile Didattica e Divulgazione INAF.
}

Corresponding author email: livia.giacomini@inaf.it

Astronomy for a better world. L'Astronomia per un mondo migliore, ovvero, la scienza come luogo di connessioni e divertimento. Non elemento magico o prodotto culturale da ammirare e basta, ma un'attività umana da condividere, con la giusta umiltà, comprendendone il linguaggio, i metodi e gli obiettivi. Questa la vision che INAF, Istituto Nazionale di Astrofisica, ha ereditato direttamente dalla IAU, Unione Astronomica Internazionale. Ė con questa impostazione che INAF si occupa dalla sua nascita di informazione, divulgazione e didattica. Tenendo conto delle enormi differenze di target, obiettivi e linguaggio di queste attività, INAF si è dotata di una Struttura per la Comunicazione articolata in diversi settori, che negli anni ha sviluppato esperienze e strumenti specifici e differenziati.

Come Media Inaf, la testata online per il grande pubblico che raggiunge oggi una media di 100.000 lettori unici mensili, o il canale YouTube MediaInaf Tv, con i suoi 5 milioni di visualizzazioni. Tra gli esempi più vicini al mondo della scuola, troviamo Edu INAF, il portale per la didattica nel campo dell'astronomia, le Olimpiadi Italiane di Astronomia, a cui nel 2018 hanno partecipato quasi 7000 studenti, e infine Martina Tremenda, la Pippi Calzelunghe dell'astrofisica che, dal 2019, porterà molte scuole nei teatri italiani.

La presenza di INAF sul territorio viene inoltre ottimizzata con il coinvolgimento crescente delle sedi locali e la realizzazione progetti didattici specifici.

Tra questi, presenteremo "Pianeti in una stanza", realizzato dall'Associazione culturale Speak Science in collaborazione con INAF-IAPS e il Dipartimento di Matematica e Fisica dell'Università degli Studi Roma Tre. Vincitore di un premio per la divulgazione scientifica di Europlanet, Pianeti in una Stanza è un kit fai da te con cui costruire un simulatore di pianeti, un piccolo proiettore sferico a basso costo realizzato in stampa $3 \mathrm{~d}$ e materiali di recupero. Utilizzato sotto una cupola, il sistema può anche funzionare come proiettore di un piccolo planetario. In distribuzione da Settembre 2018, Pianeti in una stanza è anche una comunità di appassionati di spazio e scienza che hanno deciso di costruire, usare e migliorare il sistema per far crescere la loro passione e coinvolgere le nuove generazioni. 


\section{PLANETARY SYSTEMS}




\title{
Matched Exoplanet Catalog with VO connection
}

\author{
Alei E. ${ }^{1,2} \&$ Claudi R. ${ }^{1}$ \\ ${ }^{1}$ INAF - Osservatorio Astronomico di Padova, Vicolo dell'Osservatorio 5, 35122 Padova, Italy. \\ ${ }^{2}$ Dipartimento di Fisica e Astronomia Galileo Galilei, Università di Padova, Vicolo dell'Osservatorio 3, \\ 35122 Padova, Italy.
}

\section{Corresponding author email: eleonora.alei@inaf.it}

Keywords: exoplanet catalog, exoplanets, VO connection.

The heterogeneity of observational papers makes every attempt to write a uniform catalog almost impossible. Our aim is to build a new catalog selecting the best targets whose datasets were included in one or more of the four major exoplanets online databases: NASA Exoplanet Archive [1, A], Exoplanet Orbit Database [2, B], Exoplanet Encyclopaedia [3, C] and Open Exoplanet Catalogue [4, D].

We wrote a Python code that collects and selects the most precise measurement for all interesting planetary and orbital parameters, taking into account the presence of multiple aliases for the same target. For each parameter, the code stores the corresponding reference paper link. For this reason, when the merging process is completed it could be possible to have a final dataset for each target which is not necessarily composed of consistent measurements. It is however not essential for our statistical purposes.

The code is able to download the source files from the three catalogs by use of VO ConeSearch connections to the major stellar catalogs such as SIMBAD [5] and those available in VizieR [6]. It also retrieves the compulsory user preferences through a Graphic User Interface, which allows choosing all sorts of parameter range selection. It is able to generate automatic plots that are commonly used in the exoplanetary community, but the user can retrieve and manipulate data at will.

\section{Websites:}

[A] www.exoplanetarchive.ipac.caltech.edu

[B] www.exoplanets.org

[C] www.exoplanet.eu

[D] www.openexoplanetcatalogue.com

References:

[1] Akeson et al. (2013) - PASP, Volume 125, 989-999.

[2] Wright et al. (2011) - PASP, Volume 123, Issue 902, pp. 412.

[3] Schneider et al., 2011, A 532, A79.

[4] Rein (2012) - arXiv:1211.7121.

[5] Wenger et al. (2000) - A\&AS,143,9.

[6] Cutri et al. (2003), VizieR Catalog: II/246 . CDS, Strasbourg, France. 


\title{
MAGRATHEA: 1D radiative-convective code for terrestrial atmospheres
}

\author{
Alei E. ${ }^{1,2}$, Petralia A. ${ }^{3}$, Locci D. ${ }^{3}$, Claudi R. ${ }^{1}$, Cecchi Pestellini C.. ${ }^{3}$ Ciaravella A. ${ }^{3} \&$ Micela G. ${ }^{3}$ \\ ${ }^{1}$ INAF - Osservatorio Astronomico di Padova, Vicolo dell'Osservatorio 5, 35122 Padova, Italy. \\ ${ }^{2}$ Dipartimento di Fisica e Astronomia Galileo Galilei, Università di Padova, Vicolo dell'Osservatorio 3, \\ 35122 Padova, Italy. \\ ${ }^{3}$ INAF - Osservatorio Astronomico di Palermo, P.za Parlamento 1, 90134 Palermo, Italy.
}

Corresponding author email: eleonora.alei@inaf.it

Keywords: MAGRATHEA, 1D structure code, radiative transfer, convective adjustment.

Researchers and $\mathrm{PhD}$ students belonging to an Italian collaboration among the Observatories of Padua, Palermo, and Cagliari built from scratch a 1D structure code which accounts for both radiative transfer and convective adjustment.

The radiative subroutine can calculate up and down fluxes at each atmospheric layer using absorption spectra previously adapted via the k-distribution method. The k-distribution approach is fast, flexible, and generally accurate. It reduces by orders of magnitude the computational time and the volume of data that it is usually occupied by a whole set of absorption spectra, necessary in order to retrieve the fluxes at each layer of the atmosphere. It also allows the radiative code to equally consider input absorption spectra at various pressures.

The thermo-convective subroutine was written to manage convective adjustment, following the approach proposed by Manabe \& Strickler 1964 [1], and it is coupled to the radiative counterpart.

At present time, this code allows to model Early-Earth and Mars-like cloud-free atmospheres spanning a wide range of parameters, such as pressure, temperature, and stellar irradiation.

We are working on stochastic modeling of clouds in Super Earths atmospheres as a new feature to be attached to the code. This method is based on the so-called master equation, to handle the cloud problem in an approximate way

We assume that throughout a ray the casual alternation of the gaseous/cloudy phase is a stochastic process, with Markovian transitions between states. It is then possible to define the probability of encountering a cloud deck on the line of sight and to associate the Liouville master equation to it, thus obtaining a system of coupled differential equations.

A simultaneous work is currently ongoing for what concerns thermal and photo-induced chemistry.

The actual state of the code these future upgrades and some interesting results will be presented.

\section{References:}

[1] Manabe \& Strickler (1964). Journal of the Atmospheric Sciences, 21(4), 361-385. 


\title{
A test case to understand planetary migration mechanisms from system architecture
}

\author{
Benatti S. ${ }^{1}$, Damasso M. ${ }^{2} \&$ Desidera $S .^{3}$ \\ ${ }^{1}$ INAF - OAPD. \\ 2 INAF - OATO. \\ ${ }^{3}$ INAF - OAPD and GAPS Team.
}

Corresponding author email: serena.benatti@,inaf.it

Keywords: GAPS, exoplanets, planetary migration.

The GAPS (Global Architecture of Planetary Systems) program is an Italian project for the study and characterization of exoplanets with the radial velocity method, performed with the TNG facilities. The aim of the first five years of observations was to define the planet frequency around stars with different properties and environments. The search for short period low mass planets in systems with known long period giant gaseous planets helps to define the occurrence of scaled Solar Systems, but, as shown by theoretical planetary migration models, is also a challenge, since the presence of giant bodies severely impact on the survival of small rocky planets in the same system.

In the framework of GAPS, we observed the 9 Gyr K0 star, HD164922, known to host one long period Jupiter-like planet and one temperate Neptune. Our observations show the evidence of a Super-Earth with a period of 12.46 days, recovered after a heavy mitigation of the stellar activity signal. In this talk, I will present our study with attention on the possible migration scenario for this system, characterized by an unusual architecture. 


\title{
GAPS Time Series resource and service implementation
}

\author{
Bignamini A. ${ }^{1}$, Benatti S. ${ }^{2}$, Claudi R. ${ }^{2} \&$ Molinaro M. ${ }^{1}$ \\ ${ }^{1}$ INAF-OATs. \\ ${ }^{2}$ INAF-OAPd.
}

Corresponding author email: andrea.bignamini@inaf.it

Keywords: GAPS, exoplanet modeling, time series dataset, VO connection.

The publicly available Time Series from GAPS exoplanets observations have been proposed as a use case to provide requirements to the IVOA in the effort of standardizing modelling, discovery and access to time series datasets. The interoperability effort has continued and following the current modelling draft and scenario a prototype TAP service has been built at the Italian center for Astronomical Archives to deploy the GAPS Time Series datasets. The service embeds the time series modelling solution for time axis representation and, in addition, provides a view for the recently started activity of homogeneizing the representation and discovery of exoplanetary datasets. This contribution reports current resource and service status and foreseen developments, that will focus on two main points: VO compliant serialization of the output and modelling of the exoplanetary metadata and attributes of the datasets themselves. 


\title{
Exocomets and comets
}

\author{
Capria M.T.
}

IAPS/INAF.

Corresponding author email: mariateresa.capria@iaps.inaf.it

Keywords: Exocomets, comets, exoplanetary systems.

The word "exocomet" is increasingly common in the literature on exoplanetary systems. The definition is applied to icy objects in general when they are detected, or simply suspected, in the exoplanetary systems. The real meaning of the term remains quite vague: in the solar system not all the icy bodies are necessarily a comet. And, given the incredible zoo of planet types that our instruments are discovering, are we sure that comets and exocomets are exactly the same kind of body? The real nature of the first interstellar visitor, 1'Oumuamua [1], is still unclear: could this hint to exotic types of minor bodies?

The contribution of exocomets is important not only when discussing the formation of a planetary system, but also its habitability. In order to model their formation, evolution, and contribution to the habitability of a planet we have to begin by defining what they are, and which properties, not necessarily coincident with those of our comets, they could show.

We try here to make hypotheses on possible differences in the physical properties and dynamical evolution (reservoirs) of exocomets, in view of the building of models related both to the formation and the dynamical evolution of exocomets.

\section{References:}

[1] Williams (2017) - Minor Planet Electronic Circular 2017-U181 (October 25). 


\title{
GAPS2.0: Hunting young planets
}

\author{
Claudi R. ${ }^{1}$, Benatti S. ${ }^{1} \&$ Desidera S. ${ }^{2}$ \\ ${ }^{1}$ INAF OAPD. \\ ${ }^{2}$ INAF OAPD GAPS team.
}

Corresponding author email: riccardo.claudi@inaf.it

Keywords: GAPS, exoplanets, exoplanet search.

The GAPS (Global Architecture of Planetary Systems) project gathers most of the Italian exoplanetary community. In the past 5 years our radial velocity survey with HARPS-N@TNG was focused to the search and characterization of planetary systems around stars with different characteristics ( $M$ dwarf, stars with already known planets, metal-poor stars, members of open clusters), producing important results and a robust and competitive community. Thanks to GIARPS (GIANO-B \& HARPS-N), GAPS has the opportunity to explore new perspectives on the exoplanet search: the simultaneous VIS and NIR observations can help to discriminate the nature of the radial velocity variation when observing very active stars. Taking advantage of the GIARPS capabilities, the Young Objects sub-program of GAPS2 survey focused its attention on the first stages of planetary systems formation. Recent results suggest that we could expect a large fraction of hot Jupiters around very young stars with respect to the old ones, but the current statistic is far to be complete. The observation of this kind of objects allows us to study the ongoing planet formation and to compare the planet properties at different time scales, helping to investigate the role played by the migration mechanisms, the formation sites and the orbit evolution on their observed diversity. During the first part of the program, we gained the expertise to properly model the stellar activity (Gaussian processes) starting from a suitable observing strategy, which is a crucial issue when dealing with young stars. Our target sample includes members in young associations (e.g., Taurus): some of them are known to host confirmed or candidate hot Jupiters. In this talk, I will present the main objectives of our program and the preliminary results. 


\section{ALMA highlights on protoplanetary disks}

Fedele D.

INAF, Osservatorio Astrofisico di Arcetri.

Corresponding author email: davide.fedele@inaf.it

Keywords: planetary formation, protoplanetary disks, circumstellar disks, ALMA, radiotelescopes.

Observations of protoplanetary disks can provide the physical and chemical conditions during the very early phases of planet formation and evolution. A major contribution to this field is coming from the Atacama Large Millimeter Array (ALMA). For the first time indeed, we are now able to detect and spatially resolve the emission coming from the cold disk interior. I will present some recent results obtained with ALMA observations of gas (multiple molecular species) and dust. 


\section{M stars. Activity Vs Planets}

Garrido A., Maldonado J., Affer L., Micela G., Petralia A., Maggio A. \& HADES collaboration

Corresponding author email: antonio.garrido@inaf.it

Keywords: M stars, exoplanets, HADES, radial velocity.

$\mathrm{M}$ dwarfs are currently considered ideal targets for identifying low-mass planets. However, the frequency of small planets around $\mathrm{dM}$ stars is still poorly constrained, mainly because of intense activity typical of these stars. We present the results of the HADES project at HARPS-N@TNG, focusing on the observation campaigns on GJ9440 and GJ686 including 5 years of HARPS-N radial velocity measurements as well as complementary photometry observations. We will illustrate the analysis methods, including periodograms and gaussian processes as well their limitations. The nature of the revealed periods is discussed in the context of current radial velocity programs focussed on $\mathrm{M}$ dwarfs. 


\title{
GAPS2.0: a GIANO view of the atmosphere of hot Jupiters
}

\author{
Giacobbe P. ${ }^{1}$, Bonomo A. ${ }^{1} \&$ Nascimbeni V. ${ }^{2}$ \\ ${ }^{1}$ INAF-OATo. \\ ${ }^{2}$ NAF-OAPd.
}

Corresponding author email: paolo.giacobbe@,inaf.it

Keywords: GAPS, near-infrared spectroscopy, exoplanets, planet atmospheres, transits.

The GAPS (Global Architecture of Planetary Systems) project gathers most of the Italian exoplanetary community and it is focused on the search and characterization of planetary systems. Thanks to GIARPS (GIANO-B \& HARPS-N), GAPS has the opportunity to explore new perspectives on the exoplanet characterization: We present near-infrared spectroscopy of a sample of transiting hot Jupiters with the GIANO spectrograph on the Telescopio Nazionale Galileo (TNG). GIANO offers the opportunity to probe at the high spectral resolution the planet's atmosphere simultaneously over the Y-H-J-K bands, a 21-fold increase in wavelength coverage with respect to the existing studies, e.g. based on CRIRES data. We use the highdispersion spectroscopy technique described in Brogi, Giacobbe et al. (2018 for the GIANO data and we carry out a cross-correlation analysis with different models of planetary spectra looking for the presence at high confidence of different molecular species. We discuss the prospects for detection of the individual contributions of the molecular species (e.g., water, methane, carbon dioxide, hydrogen cyanide) and for constraining the planet $\mathrm{C} / \mathrm{O}$ ratio with the GIANO data, ultimately gauging the prospects for establishing GIANO among the leading instruments for characterizing exoplanet atmospheres. 


\title{
Non-spherical dust dynamics in protoplanetary disks: how dust particle realistic shapes change the dust evolution timescales
}

\author{
Ivanovski S.L. ${ }^{1}$, Turrini D. ${ }^{2}$, Capria M.T. ${ }^{2}$, Polychroni D., Zakharov V.V., ${ }^{2,3}$, Della Corte V. ${ }^{2}$ \& Rotundi A. ${ }^{2,3}$ \\ ${ }^{1}$ INAF-Osservatorio di Trieste. \\ ${ }^{2}$ INAF-IAPS. \\ ${ }^{3}$ Università di Parthenope, Napoli.
}

\section{Corresponding author email: stavro.ivanovski@inaf.it}

Keywords: planetary formation, protoplanetary disks, circumstellar disks, dust dynamics.

The dust evolution and dynamics studied through the observational capabilities of SKA will prompt the need of refined theoretical models that can explain features of the dust evolution processes previously impossible to observe. Already, high-resolution observations of protostellar discs from the infrared to sub-mm instruments at large telescopes (as LBT and VLT) showed that gas and dust, while coupled through drag, can undergo divergent dynamical evolution.

We propose a novel cross-disciplinary study that will transfer know-how and methods derived from recent advancements in the field of Solar System studies to the study of circumstellar discs.

Since the theoretical modeling of the dust dynamical evolution in the Epstein regime is not available in the most numerical codes and tools we will provide a unique perspective of the dust evolution in circumstellar discs and explore how dust can be injected and sustained in a vertically extended region spanning several AU from the mid-plane under comparatively gas-poor environment. [1] presents UV and IR observations of H2 and CO in the young T Tauri system RY Lupi and, combining these inner disk gas tracers for the first time, allows one to derive a mapping of the radial structure in the region of the system, where protoplanets may form. Such young protoplanets can be responsible for the prominent gaps in the dust continuum emission as suggested by the high-resolution images of ALMA [2,3] showing with high precision the spatial extent of these protoplanetary disks both vertically and radially. Based on the expertise on modeling non-spherical dust dynamics in rarefied gas field in cometary environment [4,5], we report results on the dynamics of nonspherical particles in protoplanetary disks when the gas flow is in Epstein regime, i.e. in rarefied gas field.

References:

[1] Arulanantham et al. (2018) - ApJ, 855.

[2] ALMA Partn. et al. (2015).

[3] Isella et al. (2016).

[4] Ivanovski et al. (2017), Icarus 282.

[5] Ivanovski et al. (2017), MNRAS. 


\title{
Dynamical Models to Explain Double Debris Belts Systems and Comparison with SPHERE Observations
}

\author{
Lazzoni C. ${ }^{1}$, Desidera S. ${ }^{2} \&$ Marzari F. \\ ${ }^{1}$ INAF-OAPD, Università di Padova. \\ 2 INAF-OAPD. \\ ${ }^{3}$ Università di Padova.
}

Corresponding author email: cecilia.lazzoni@,oapd.inaf.it

Keywords: exoplanetary systems, debris disks, planetary system formation, SPHERE.

A large number of extra-solar systems harboring a debris disk show evidence for a double belt architecture, as in the solar system. We observed several tens of these systems with SPHERE as for example HR8799. One likely hypothesis for explaining the gap between the belts is the presence of one or more planets dynamically carving it. This work aims to investigate this scenario in systems with two components debris disks. All the targets in the sample were observed with the SPHERE instrument which performs high-contrast direct imaging. Positions of the inner and outer belts were estimated by SED fitting of the infrared excesses or from resolved images of the disk. Very few planets have been observed so far in debris disks gaps and we intended to test if such non-detections depend on the observational limits of the present instruments. This aim is achieved by deriving theoretical predictions of masses, eccentricities and semi-major axes of planets able to open the observed gaps and comparing such parameters with detection limits obtained with SPHERE. The relation between the gap and the planet is due to the chaotic zone around the orbit of the planet. The radial extent of this zone depends on the planet-star mass ratio, on the semi-major axis and on the eccentricity of the planet and it can be estimated analytically. We apply the formalism to the case of one planet on a circular or eccentric orbit. We then consider multi-planetary systems: 2 and 3 equal-mass planets on circular orbits and 2 equal-mass planets on eccentric orbits in a packed configuration. We then compare each couple of values $(\mathrm{M}, \mathrm{a})$, derived from the dynamical analysis of single and multiple planetary models, with the detection limits obtained with SPHERE. Our results show that the apparent lack of planets in gaps between double belts could be explained by the presence of a system of two or more planets possibly of low mass and on an eccentric orbit whose sizes are below the present detection limits. 


\title{
A Data Model for discovery and description of Exoplanetary Systems Datasets
}

\author{
Molinaro M. ${ }^{1}$, Alei E. ${ }^{2,5}$, Benatti S. ${ }^{2}$, Bignamini A. ${ }^{1}$, Bonnarel F. ${ }^{3}$, Damasso M. ${ }^{4}$, Louys M. ${ }^{3}$, \\ Maris M. ${ }^{1} \&$ Nascimbeni $V^{2,5}$ \\ ${ }^{1}$ INAF - OATs. \\ ${ }^{2}$ INAF - OAPd. \\ ${ }^{3}$ CDS (CNRS, France). \\ ${ }^{4}$ INAF - OATo. \\ ${ }^{5}$ Dip. Fisica e Astronomia - Università di Padova.
}

Corresponding author email: marco.molinaro@,inaf.it

Keywords: Exoplanets, exoplanetary systems, exoplanet catalog, exoplanet dataset.

Datasets from observations, simulations and catalogues of exoplanetary systems are growing in number and details. Tackling interoperability of these data and finding a common, shared way to describe them would be a benefit to the whole community. Starting this effort now has the advantage of working while the field is relatively new, thus not incurring in the risk of having to rework major loads of existing data. An interoperable data model, following the guidelines and technical solutions proposed by the IVOA, could work both in terms of dataset discovery, i.e. finding relevant datasets to be used on scientific production, and with respect of datasets description, i.e. adding proper metadata to let datasets be meaningful to the community and re-usable by third party researchers. An effort has been started in this direction under the H2020 ASTERICS umbrella and is proving to be of interest at global level. The data model ideally picks up model components from IVOA specifications, either existing or under investigation, and attach new ones where needed. It covers a first set of requirements and considerations and takes into account aspects like the observations of exoplanetary systems, the usage of existing exoplanets catalogues, the investigation of atmospheres of confirmed exoplanets and the simulation of exoplanet's atmospheres devoted to characterize exoplanets habitability.

This contribution reports the history of this activity, its current status and its goals. 


\section{La missione spaziale ARIEL}

Pace E.

on the behalf of the ARIEL Consortium.

Corresponding author email: emanuele.pace@unifi.it

Keywords: ARIEL, space missions, extrasolar planets, atmospheres.

"ARIEL is the Atmospheric Remote-Sensing Infrared Exoplanet Large-survey, which was down-selected for a Phase-B1 study as an ESA M4 candidate mission in 2018. Its aim is to characterise the atmospheres of around 500 warm-hot exoplanets orbiting nearby bright stars.

Using the differential technique of transit and eclipse spectroscopy, ARIEL will obtain transmission and/ or emission spectra of the atmospheres of a large and diverse sample of known exoplanets covering a wide range of masses, densities, equilibrium temperatures, orbital properties and host-star characteristics. The instantaneous spectral coverage of ARIEL is unique in its breadth, spanning from visible to thermal infrared. This broad range opens up the possibility to study exoplanets with physical temperatures ranging from several hundred to over a few thousand Kelvin. Importantly the visible waveband provides an essential means by which to monitor and subsequently correct for the effects of activity of the host star, which could otherwise introduce significant uncertainty into the final exoplanet spectrum and its interpretation.

Scientific goals and the payload structure will be presented along with the Italian contribution. 


\title{
Evolution of protoplanetary disks in Open Clusters. Material mixing in the protoplanetary matter after the perturbation of passing-by stars.
}

\author{
Pinto L.D. ${ }^{1}$, Magni G. ${ }^{1}$, Capaccioni F. ${ }^{1}$ \& Capuzzo-Dolcetta R. ${ }^{2}$ \\ ${ }^{1}$ INAF-IAPS. \\ ${ }^{2}$ Sapienza, Università di Roma.
}

Corresponding author email: luis.pinto@,inaf.it

Keywords: planetary formation, protoplanetary disks, circumstellar disks, planetary evolution, open clusters.

Recent detection of both planetary systems and protoplanetary gaseous matter around stars in Open Clusters opened new perspectives in the theory of planet formation. The secular processes of alpha-viscosity evolution ([1]), so far deeply investigated, under certain conditions, may bring protoplanetary disks to condensate and to form planets. Such evolution may lead to different final configurations if protoplanetary disks are embedded in a more complex environment like Open Clusters: a context in which they may be substantially perturbed by the interaction with other field stars. Modelling of such complex systems is important as recent dynamical models have pointed out the high probability of the evolution of exoplanetary systems in Open Clusters.

We investigate with our own Smooth Particle Hydrodynamic (SPH) code the evolution of a self-gravitating disk around a single star, interacting with several planetary cores embedded in it. We already investigated the dynamical effects of passing by stars, where a series of numerical simulations for an isothermal model has been described. A subsequent step in the code development is the addition of the thermal evolution of the gas by considering the radiative transport through matter.

In the meantime, we focus our attention on the mass transport and material mixing within the protoplanetary disk due to the fly-by with a passing by star. The evolution of the disk is studied as a function of several parameters, e.g. the masses of the two stars, their relative velocity, the impact factor, the mass of the protoplanetary disk, etc. The talk will describe the model used and the effects the various parameters have in the subsequent evolution of the protoplanetary disk.

\section{References:}

[1] Shakura \& Sunyaev (1973) - Astronomy \& Astrophysics. 


\title{
ARIEL and the quest to understand planetary diversity
}

\author{
Turrini D. ${ }^{1}$, Oliva F. ${ }^{1}$, Migliorini A. ${ }^{1}$, Politi R. ${ }^{1}$, Fonte S. ${ }^{1}$, Fedele D. ${ }^{2}$, Molinari S. ${ }^{1}$ \& Schisano E. ${ }^{1}$ \\ ${ }^{1}$ INAF - Istituto di Astrofisica e Planetologia Spaziali. \\ ${ }^{2}$ INAF - Osservatorio Astronomico di Arcetri.
}

Corresponding author email: diego.turrini@inaf.it

Keywords: ARIEL, extrasolar planets, atmospheres, planetary formation, spectral signatures.

ARIEL (Atmospheric Remote-Sensing Infrared Exoplanet Large-survey), ESA's fourth M-class space mission, will investigate the atmospheric composition of hundreds of exoplanets in close orbits around bright stars and will search for the spectral signatures of tens of molecules formed by elements spanning all main cosmochemical classes, from the most refractory ones to the most volatile ones [1]. The strong tidal interactions between the planets of ARIEL's sample and their host stars, together with their high atmospheric temperatures, result in a strong coupling between their interior and their atmospheric compositions and make these planets unique natural laboratories to study the processes of planetary formation and migration and understand the origins of the extreme planetary diversity in our galaxy [2]. To fully take advantage of the opportunity that will be offered by ARIEL's observations, however, it will be necessary to integrate the unique know-how and methods provided by a range of disciplines spanning from star formation to planetary formation, and from the study of circumstellar disks to that of planetary atmospheres in the Solar Systems. Within the ARIEL Consortium, the ARIEL "Planet Formation" Working Group is performing a number of activities aiming at achieving this goal: in this talk we will provide an overview of the Italian contributions to such efforts.

\section{References:}

[1] Tinetti et al. (2018) - A chemical survey of exoplanets with ARIEL. Experimental Astronomy 46, 135-209. DOI: $10.1007 / \mathrm{s} 10686-018-9598-x$.

[2] Turrini et al. (2018). The contribution of the ARIEL space mission to the study of planetary formation. Experimental Astronomy 46, 45-65. DOI: 10.1007/s10686-017-9570-1. 


\title{
ARTECS and ExoplAn3T: toward a synergy between two exoplanetary tools
}

\author{
Zinzi A. ${ }^{1}$, Maris M. ${ }^{2}$, Alei E. ${ }^{3}$, Murante G. ${ }^{1}$, Silva L. ${ }^{1}$, Turrini D. ${ }^{4}$, Verrecchia F. ${ }^{5}$ \& Vladilo G. ${ }^{1}$ \\ ${ }^{1}$ Space Science Data Center - ASI. \\ ${ }^{2}$ INAF - Osservatorio Astronomico di Trieste. \\ ${ }^{3}$ INAF - Osservatorio Astronomico di Padova; Dipartimento di Fisica e Astronomia Galileo Galilei, \\ Università di Padova. \\ ${ }^{4}$ INAF-IAPS. \\ ${ }^{5}$ Space Science Data Center - ASI; INAF-OAR.
}

Corresponding author email: angelo.zinzi@ssdc.asi.it

Keywords: ARTECS, ExoplAn3T, exoplanets, software facilities, climate models, observational database.

ARTECS (http://wwwuser.oats.inaf.it/exobio/climates/) and ExoplAn3T (https://tools.ssdc.asi.it/exoplanet) are two innovative software facilities independently developed by Astronomical Observatory of Trieste (INAF-OATS) and Space Science Data Center (SSDC-ASI), respectively, for exoplanetary sciences. ARTECS is a repository of climate models of terrestrial exoplanets, while ExoplAn3T provides access to observational databases with an interface designed and optimized for the study of exoplanetary systems as a whole. The synergistic use of the two facilities offers new possibilities for exoplanetary studies taking advantage of existing observational data. In order describe the capabilities offered by this approach we will present two different use cases, the first one related to the communication from ARTECS to ExoplAn3T and the second one about the inverse link:

1) the user can look for precise climate conditions, obtaining as output a well-determined class of exoplanets, based on the type of host star, semi-major axis of the planet and other planetary and orbital parameters. Once the output is ready it can be sent to the query mask of ExoplAn3T that will return as a final result a list of exoplanetary systems with at least one planet fulfilling the conditions to possess the desired climate.

2) the user will query exoplanet catalogues by means of ExoplAn3T, subsequently focusing on the best promising exosystems. The final step is represented by the forwarding of the observed exoplanetary parameters from ExoplAn3T to ARTECS, allowing to find typical climates for the terrestrial planets in the sample.

The goal of the present work is to establish a communication protocol between the two tools for the end of 2019, taking advantage from the long-lasting experience of the two working groups in the field of scientific data management and interoperability, particularly in Virtual Observatory (VO) activities, thus providing the community with a new and useful scientific tool. 


\section{HABITABILITY AND ASTROBIOLOGY}




\title{
Supermassive black holes and planetary habitability
}

\author{
Balbi A. ${ }^{1}$, Kovacevic A.B. ${ }^{2}$, Tombesi F. ${ }^{1,3,4}$ \& Wislocka A.M. ${ }^{5}$ \\ ${ }^{1}$ Dipartimento di Fisica, Università degli Studi di Roma "Tor Vergata", Italy. \\ ${ }^{2}$ Department of Astronomy, Faculty of Mathematics, University of Belgrade, Serbia. \\ ${ }^{3}$ Department of Astronomy, University of Maryland, USA. \\ ${ }^{4}$ NASA/Goddard Space Flight Center, USA. \\ ${ }^{5}$ Institute for Astro \& Particle Physics, University of Innsbruck, Austria.
}

Corresponding author email: amedeo.balbi@roma2.infn.it

Keywords: Galactic habitability, high energy events, biosphere.

It is generally thought that the habitability of a planet is strongly influenced by the amount of ionizing radiation incident on its atmosphere and surface. While the host star is usually the prevalent origin of such high-energy radiation, previous studies have also considered the effect on a hypothetic biosphere of other possible sources, in particular with regard to potentially catastrophic transient events such as nearby gamma ray bursts or supernova explosions. Here we consider a phenomenon whose astrobiological consequences are much less explored, i.e. the production of X-ray and extreme ultraviolet (XUV) radiation during the peak of the active phase of supermassive black holes. In particular, we studied [1] how the activity of the supermassive black hole at the center of the Milky Way, known as Sagittarius A* (Sgr A*), may have affected the habitability of Earth-like planets in our Galaxy. Our results show for the first time that the combined effect of atmospheric loss and of the direct biological damage to surface life was probably significant during the AGN phase of Sgr $\mathrm{A}^{*}$, possibly hindering the development of complex life within a few kiloparsecs from the galactic center. As a further investigation in this direction, we included the contribution of XUV radiation from nearby AGNs and estimated its overall impact on known rocky exoplanets in our galaxy [2].

References:

1. Balbi \& Tombesi (2017) - Scientific Reports 7, 16626.

2. Wislocka et al. (2018) - submitted. 


\title{
Nitrogen incorporation into aromatic rings in extraterrestrial environments: toward the formation of pyridine and nucleobases
}

\author{
Balucani N., Skouteris D., Caracciolo A., Casavecchia P. \& Rosi M. \\ DCBB - Università degli Studi di Perugia. \\ DICA - Università degli Studi di Perugia. \\ Master-Up, Perugia.
}

Corresponding author email: nadia.balucani@unipg.it

Keywords: astrochemistry, gas-phase reactions, molecules, experimental.

Gas-phase reactions involving atomic nitrogen in the ground $4 \mathrm{~S}$ and first excited 2D electronic states with simple hydrocarbons or hydrocarbon radicals lead to the formation of prebiotic N-containing organic molecules. These reactions are now active in the upper atmosphere of Titan (a massive moon of Saturn) and might have played an important role in nitrogen fixation in the primitive upper terrestrial atmosphere. Their products (nitriles, imines and $\mathrm{N}$-containing organic radicals) are the precursors of larger $\mathrm{N}$-containing molecules, which form the dense haze aerosols that completely cover Titan. If anything similar to Titan's haze has ever existed on primitive Earth, it is reasonable to imagine that, once deposited on the surface of the oceans, further chemical evolution might have transformed these molecules into aminoacids and nucleobases, the molecular building blocks of living entities [1].

$\mathrm{n}$ our laboratory, we have investigated the reactions of $\mathrm{N}(2 \mathrm{D})$ with several aliphatic hydrocarbons common in the atmosphere of Titan [2-4] and we have verified that products containing a novel C-N bond are formed, thus corroborating the suggestion that these gas-phase reactions allow for nitrogen fixation in organic compounds. More recently, we have turned our attention to N(2D) reactions with aromatic hydrocarbons, namely benzene and toluene (both present in the upper atmosphere of Titan). The main results will be illustrated in this contribution, but we anticipate here that the most striking result is the formation of an aromatic compound, pyridine, where $\mathrm{N}$ is directly incorporated into the aromatic ring [5].

\section{References:}

[1] Balucani (2012) - Chem. Soc. Rev., 41, 5473-5483.

[2] Balucani et al. (2009) - J. Phys. Chem. A, 113, 11138.

[3] Balucani et al. (2010) - Faraday Discuss., 147, 189.

[4] Balucani et al. (2012) - J. Phys. Chem. A, 116, 10467.

[5] Balucani et al. (2018). Astrobiology, in preparation. 


\title{
An innovative setup to investigate by remote sensing growth and photosynthetic performances of cyanobacteria exposed to exo-earths simulated environments
}

\author{
Battistuzzi M. ${ }^{1,5}$, Cocola L. ${ }^{2}$, Claudi R. ${ }^{3}$, Alei E. ${ }^{3,4}$, Poletto L. ${ }^{2}$, Morosinotto T. ${ }^{5} \&$ La Rocca N. ${ }^{5}$ \\ ${ }^{1}$ Centro di Ateneo di Studi e Attività Spaziali "Giuseppe Colombo" - CISAS, Via Venezia 15, 35131, Padova. \\ ${ }^{2}$ CNR-IFN, Via Trasea 7, 35131, Padova. \\ ${ }^{3}$ INAF - Astronomical Observatory, Vicolo Osservatorio 5, 35122, Padova. \\ ${ }^{4}$ Dept. Physic and Astronomy Galileo Galilei, Padova Univ., Vicolo Dell’Osservatorio 3, I-35122 Padova. \\ ${ }^{5}$ Dept. Biology, Padova Univ., Via U. Bassi 58/b, 35131, Padova.
}

Corresponding author email: mariano.sk8er@gmail.com

Keywords: astrobiology, microbiology, photosymthesis, experimental.

During recent years, by means of space and ground-based observations, more than 3800 new exoplanets[1] have been discovered, many of which are terrestrial-like planets orbiting the Habitable Zone of their host stars, enhancing our hope to find life elsewhere in the universe. Due to the great distances separating us from these planets, any study regarding their habitability must be made through remote sensing techniques or in laboratory, by means of environmental condition simulations. Thanks to the collaboration between INAF, CNR-IFN and the department of Biology of Padova, a Star Light Simulator (SLS) and an Atmosphere Simulator Chamber (ASC) have been built. Using these devices, we study oxygenic photosynthetic microorganisms under simulated environmental conditions of terrestrial-like exoplanets orbiting M-type stars, to understand if they could maintain their oxygenic photosynthetic activity and furthermore impact on primeval atmospheres lacking oxygen. Testing these conditions requires light, temperature, pressure and atmospheric composition conditions that can be extremely different from terrestrial ones and imposes us to seal the ASC and assess the physiological responses of the cultures only at the beginning and at the end of the experiments. To overcome this issue, here we present a novel experimental setup to follow by remote sensing the growth and photosynthetic activity of oxygenic photosynthetic microorganisms by means of reflectivity, spectroscopic and fluorescence measurements, respectively.

\section{References:}

[1] https://exoplanets.nasa.gov/ 


\title{
Desert cyanobacteria under planetary simulations: towards future astrobiology experiments in space
}

\author{
Billi D. ${ }^{1} \&$ Balbi A. ${ }^{2}$ \\ ${ }^{1}$ University of Rome Tor Vergata, Department of Biology, Rome Italy. \\ ${ }^{2}$ University of Rome Tor Vergata, Department of Physics, Rome Italy.
}

Corresponding author email: billi@uniroma2.it

Keywords: astrobiology, cyanobacteria, survaiability, experimental.

The occurrence of extremophiles thriving in hot and cold deserts and in sub-glacial lakes, considered as planetary field analogues for Mars and icy moons of Jupiter and Saturn, is reshaping the boundaries of habitability [1]. New insights into what makes a planet or an icy-moon habitable are provided by experiments in which extremophiles are exposed to simulations of planetary and deep space conditions [2].

Under laboratory conditions, desert strains of Chroococcidiopsis tolerate at least 4 years of air-drying, up to $13 \mathrm{~kJ} / \mathrm{m}$ of UVC irradiation $15 \mathrm{kGy}$ of X-rays and $12 \mathrm{kGy}$ of $\gamma$-radiation. When irradiated in the dried state, they exhibit an enhanced radiation resistance, being able to cope with $30 \mathrm{~kJ} / \mathrm{m}$ of a simulated Martian UV flux, $24 \mathrm{kGy}$ of $\gamma$-radiation and $2 \mathrm{kGy}$ of Fe ions $[3,4]$. Recently, these cyanobacteria were exposed in the dried, ametabolic state to ground-based simulations of space and Martian conditions, as well as to space and Marslike conditions in low Earth orbit. When exposure did not exceed repair capabilities upon rewetting, data were inferred on physico-chemical constraints of life. When the accumulated damage exceeded survival potential, the biomarkers persistence contributed to identify biosignatures for searching life [4].

In the contest of the ASI project "Life in Space", desert strains of Chroococcidiopsis will be exposed in the hydrated, metabolically active state to laboratory simulations of Mars and icy-moon conditions, as well as to an infra-red source for photosynthesis. Deciphering the molecular mechanisms of their adaptation will enhance our knowledge on the adaption potential of life and will provide a valuable requisite for future astrobiology experiments to be carried out on cubesats or/and outside the deep space gateway. The characterization of the spectral features of the photosynthetic pigments synthetized under non-Earth-simulations will contribute to identify spectroscopic surface biosignatures on exoplanets [5].

\section{References:}

[1] Martins et al. (2017) - Space Sci. Rev., 209, 43-81.

[2] Cottin et al. (2017) - Space Sci. Rev., 209, 83-181.

[3] Billi (2018) - Int. J. Astrobiology, 1-7.

[4] de Vera et al. (2019) - Springer International Publishing, pp 111-122.

[5] Schwieterman et al. (2018) - Astrobiology 18, 663-708. 


\title{
Photoprocessing of formamide ice: route towards prebiotic chemistry in space
}

\author{
Corazzi M.A. ${ }^{1,2}$, Brucato J.R. ${ }^{2} \&$ Poggiali G. ${ }^{1,2}$ \\ ${ }^{1}$ Department of Physics and Astronomy, University of Florence, via Sansone 1, 50019 Sesto Fiorentino, Italy. \\ ${ }^{2}$ INAF-Astrophysical Observatory of Arcetri, L.go E. Fermi 5, 50125 Firenze, Italy.
}

Corresponding author email: corazzi@arcetri.astro.it

Keywords: prebiotic chemistry, ices, photoprocessing, experimental.

The chemical reactions of molecules containing $\mathrm{H}, \mathrm{C}, \mathrm{N}$ and $\mathrm{O}$ are considered a plausible pathway for synthesis of biomolecules under prebiotic conditions. Formamide (HCONH2) is the simplest molecule containing the peptide linkage [1] first detected in the gas phase in Orion-KL and SgrB2 [2,3]. It is plausible that it is initially adsorbed on the surface of the grains within these regions of star formation and then sublimates as a consequence of the radiation coming from the stars. Interpretation of observations can benefit of laboratory activities where it is possible to study formamide under simulated space conditions such as UV irradiation and low temperature. Here we report two laboratory analyses: the first concerns the study of formamide photo stability under UV irradiation when adsorbed on space relevant minerals at $63 \mathrm{~K}$ and in high vacuum regime. The second analysis is aiming to investigate Temperature Programmed Desorption of pure formamide and after UV irradiation.

These laboratory results will support observational data and improving our understanding of the role of grain surface in enriching the chemistry in space.

\section{References:}

[1] Saladino (2012) - COSPAR.

[2] Turner (1991) - ApJS.

[3] Nummelin (1998) - ApJS. 


\title{
The Volatile Composition of Comets as revealed by near-IR High-Resolution Spectroscopy
}

\author{
Faggi S. ${ }^{1}$, Mumma M.J. ${ }^{1}$, Villanueva G.L. ${ }^{1}$, Paganini L. ${ }^{1,2} \&$ Lippi M. ${ }^{1,3}$ \\ ${ }^{1}$ NASA Goddard space Flight Center, Greenbelt, MD 20771, USA. \\ ${ }^{2}$ Catholic University of America, Washington DC, 20064, USA. \\ ${ }^{3}$ American University, Washington DC, 20016, USA.
}

Corresponding author email: sara.faggi@,nasa.gov

Keywords: comets, molecules, high resolution spectroscopy.

The importance of studying water and organic molecules in space is related to their biological relevance. Comets are considered the most pristine bodies of the solar system and their investigation through cosmogonic parameters provides important information on native conditions in the solar nebula. Our knowledge about infant stages of our planetary system is still fragmentary and cometary nuclei retain the least processed material from that era. Investigation of comets composition, based on cosmogonic indicators, is essential for testing models of Solar System formation, for assessing cometary delivery of organic compounds to the early Earth, and for addressing the origin of water on Earth (Mumma \& Charnley 2011). High resolution spectroscopy with long-slit echelle spectrometers is powerful method for ground- based IR surveys. In the infrared spectral region of about (3-5 m), trace volatiles, including $\mathrm{CO}, \mathrm{H}_{2} \mathrm{CO}, \mathrm{CH}_{3} \mathrm{OH}, \mathrm{CH}_{4}, \mathrm{C}_{2} \mathrm{H}_{2}, \mathrm{C}_{2} \mathrm{H}_{6}, \ldots$ are sampled simultaneously with $\mathrm{H}_{2} \mathrm{O}$ (Mumma et al. 2003; DiSanti et al. 2008). Symmetric species $\left(\mathrm{CH}_{4}, \mathrm{C}_{2} \mathrm{H}_{6}\right.$, etc.) lack a permanent dipole moment and so are not active in pure rotational emission (radio, submillimeter), and their excited electronic states are usually pre-dissociated. Thus, they are uniquely sensed through their vibrational emissions at IR wavelengths, usually excited by solar fluorescence (Di Santi et al. 2006; Villanueva et al. 2011, 2013). Today, advances in sensitive high-resolution spectrometers and analytical spectroscopic tools continue to open new paths in the exploration of comets and planetary atmospheres. In particular, high-resolution infrared echelle-spectrometers now permit exploration of compositions for a broad range of planetary sources with unprecedented precision. By providing major increases in spectral resolving power, spectral grasp, and instrumental sensitivity, the emergence of a new class of high-resolution IR echelle spectrometers offers the next advance in this field. 


\title{
3D Simulations of Exoplanetary Atmosphere
}

\author{
Galuzzo D. ${ }^{1}$, Berrilli F. ${ }^{1}$, Cagnazzo C. ${ }^{2} \&$ Giovannelli L. ${ }^{3}$ \\ ${ }^{1}$ Università Tor Vergata. \\ ${ }^{2}$ Istituto di Scienze Marine - CNR. \\ ${ }^{3}$ Università Tor Vergata.
}

Corresponding author email: daniele.galuzzo@gmail.com

Keywords: High resolution spectroscopy, Extremely Large Telescpe, instrumentation, exoplanets.

Three-dimensional General Circulation Models (GCMs) are at the moment, the best available tools for investigating and predict the properties of the exo-atmospheres of Earth-like planets orbiting nearby stars. As a case study, we investigate the climate of the tidally locked Earth-like planet orbiting around Proxima Centauri via the 3-D GCM PlaSim and the 1-D radiative transfer model uvspec. A planetary preindustrial atmosphere, circular orbit and null axial tilt are assumed. The model output includes the atmospheric dynamics, surface temperature and the presence of liquid water, as well as reflective and emission spectra of the planet at high resolution. Our tool can effectively retrieve atmospheric fingerprints of Earth-like planets of nearby systems, giving clues on the habitability of such planets, and has been used to set observational limits with space-born (e.g., JWST) and ground-based telescopes. 


\title{
Is primordial atmosphere a key to organic matter delivery to early Earth?
}

\author{
Longo S. ${ }^{1} \&$ Micca Longo G. ${ }^{1}$ \\ ${ }^{1}$ Dipartimento di Chimica, Università degli Studi di Bari.
}

Corresponding author email: savino.longo@uniba.it

Keywords: Earth early atmosphere, meteorites, modelling.

A model of the atmospheric entry of sub-mm grains is applied to the study of the effect of different hypothesis of a primordial Earth atmosphere on the heating during the atmospheric entry, in the context of organic matter delivery. We demonstrate that 1 . the total gas budget of the atmosphere is not highly relevant as far as the determination of the heating associated with slowing to subsonic speed is concerned 2. accordingly, light components which are expected to be present in a primordial atmosphere and even more abundant in the upper one, may be the primary components to be considered in the evaluation of momentum and heat transfer in such scenarios, finally 3 . lighter components lead to lower heating for the same momentum transfer. Calculations are performed with model spherical, uniform grains of "conventional" composition as well for the recently proposed "white soft mineral" (WSM) grains for which chemical decomposition cooling is also important. Strong reduced heating is obtained for an upper atmosphere rich in light components, showing that the composition of the primordial Earth atmosphere may be the key issue in the quantification of the delivery and pre-biotic role of thermolabile organic matter enclosed in sub-mm extraterrestrial grains. 


\title{
Exoplanet atmospheres with HIRES, an optical-NIR high resolution spectrograph for the ELT
}

\author{
Marconi A. on behalf of the HIRES Consortium
}

Dipartimento di Fisica e Astronomia, Università di Firenze INAF-Osservatorio Astrofisico di Arcetri on behalf of the HIRES Consortium.

Corresponding author email: alessandro.marconi@unifi.it

Keywords: exoplanet, atmosphere, modelling.

HIRES is an optical-infrared High-Resolution Spectrograph for ESO Extremely Large Telescope, for which the phase A study has just been completed by a large international consortium and the construction is expected to start in 2019. The baseline design is modular fiber-fed cross-dispersed echelle spectrograph with two ultrastable spectral arms providing a simultaneous spectral range of 0.4-1.8 $\mu \mathrm{m}$ at a spectral resolution of $\sim 100,000$. ELT-HIRES will have several, interchangeable observing modes including the possibility of a variable number of apertures hence a small multi object capability. The top science cases will be the detection of life signatures from exoplanet atmospheres, tests on the stability of Nature's fundamental couplings, the direct detection of the cosmic acceleration. In this talk, after a brief description of the instrument, I will focus on the top priority science case for HIRES, the study of exoplanet atmospheres and the search for biosignatures in Earth analogues. 


\title{
Amino acids from photo- and thermo-processing of extraterrestrial ices: a possible source for further prebiotic chemistry
}

\author{
Modica P. ${ }^{1}$, Martins Z. ${ }^{2}$, Meinert C. ${ }^{3} \&$ d'Hendecourt L. ${ }^{4}$ \\ ${ }^{1}$ CNRS/LPC2E, Orléans (France). \\ ${ }^{2}$ CQFM-IN and IBB, Lisboa (Portugal). \\ ${ }^{3}$ Institut de Chimie, Nice (France). \\ ${ }^{4}$ PIIM, Marseille (France).
}

Corresponding author email: paola.modica@cnrs-orleans.fr

Keywords: Prebiotic chemistry, ices, photoprocessing, experimental.

Laboratory experiments that simulate the photo- and thermo-processing of extraterrestrial ices always lead to the formation of semi-refractory organic residues [1,2]. These residues can be considered as laboratory analogs for the primitive organic matter incorporated into comets and asteroids. Many specific organic molecules have been detected in them [3,4]. Here we focus on amino acids because of their possible relevance to further prebiotic chemistry on Earth. We compare the amino acid content and distribution measured in organic residues produced in our experiments to those observed in CM chondrites presenting an increasing degree of aqueous alteration [5], a process thought to impact amino acid chemistry.

Moreover, the discovery of meteoritic amino acids with chiral asymmetry (enantiomeric excesses of the left form) has suggested that extraterrestrial organic materials may have contributed to prebiotic chemistry and directed the initial chiral asymmetry that further led to homochirality of amino acids on living organisms [6]. A proposed mechanism for the origin of chiral asymmetry in meteoritic amino acids involves an asymmetric photochemistry of extraterrestrial ices by chiral (left or right circularly polarized) UV light [7]. We have performed the asymmetric synthesis of amino acids on extraterrestrial ice analogs and measured the induced enantiomeric excesses [8].

We present our experimental results and discuss their astrophysical implications. These results support the idea of a general formation process for amino acids from photo- and thermo-processing of extraterrestrial ices as an important source for the inventory of amino acids in the early solar system.

\section{References:}

[1] Allamandola et al. (1988) - Icarus.

[2] de Marcellus et al. (2017) - MNRAS.

[3] Meinert et al. (2012) - Chem. Plus. Chem.

[4] Meinert et al. (2016) - Science.

[5] Modica et al. (2018) - ApJ.

[6] Glavin et al. (2012) - M\&PS.

[7] Fukue et al. (2010) - OLEB.

[8] Modica et al. (2014) - ApJ. 


\title{
Exoplanetary Exosphere and Exospheric tail
}

\author{
Mura A. ${ }^{1}$, Benatti S. ${ }^{2}$, Locci D. ${ }^{3}$, Micela G. ${ }^{3}$, Milillo A. ${ }^{1}$, Petralia A. ${ }^{3} \&$ Vladilo G. ${ }^{4}$ \\ ${ }^{1}$ INAF-IAPS. \\ 2 INAF-OAPD. \\ ${ }^{3}$ INAF-OAPA. \\ ${ }^{4}$ INAF-OATS.
}

Corresponding author email: alessandro.mura@gmail.com

Keywords: exoplanets, exosphere, modelling.

In this study we aim to provide a model for exosphere and exoplanetary tails. In particular, we want to improve and exploit a new method to characterize an extrasolar planet in terms of a fundamental question about habitability: how stable is its atmosphere? The approach consists in finding, in the spectra of the transit curves, "signatures" of exospheric tails, whose characteristics provide information on the stability of the atmosphere. To help the interpretation of the observations, here we present a 3D model developed to simulate the columnar density exosphere and magnetospheric, from which the transit curves can be obtained in appropriate bands. With the number of known extrasolar planets doubling every 30 months, it is necessary to count on a growing "exoplanetary community" and with sophisticated and shared modeling tools. Therefore, the ultimate goal is not only to develop a model that can be used within the proposed project but to make this model freely accessible online, to give the vast scientific community a tool to make the most of every observation of transit. Through the model, in combination with observations, it will be possible to obtain the measurement of the atmospheric loss rate by detecting the characteristics of the exosphere tails. This will increase our current knowledge on the interaction of stellar wind with planets outside the solar system, on indicators for the stability of the atmosphere and the potential habitability, on the protection of the extrasolar planets from the stellar winds, important for the possibility of life; on the instrumental requirements for the observation of those conditions of potential habitability. 


\title{
Bistability in a climate model for rocky planets
}

\author{
Murante G. ${ }^{1}$, Provenzale A. ${ }^{2}$, Vladilo G. ${ }^{1}$, Silva L. ${ }^{1}$, Maris M. ${ }^{1}$, Palazzi E. ${ }^{3} \&$ Taffoni G. ${ }^{1}$ \\ ${ }^{1}$ INAF- Osservatorio di Trieste \\ ${ }^{2}$ CNR-IGG Pisa \\ ${ }^{3}$ CNR-ISAC Torino
}

\section{Corresponding author email: Giuseppe.Murante@inaf.it}

Keywords: exoplanets, habitability, climate, modelling.

The circumstellar habitable zone is defined as the range of distances from the central star where a planet can host liquid water, or, more strictly, the presence of metazoans (see Silva et al. 2017). Classically, its boundaries are set using the onset of a runaway greenhouse effect (inner boundary) and the maximum CO2 greenhouse (outer one). However, the climate of a planet heavily influences its surface temperature and thus its habitability. Using out ESTM model (Earth-like Surface Temperature Model; Vladilo et al 2015), we are able to estimate the impact of a planet's climate on its surface temperature. ESTM couples a classic EnergyBalance Model (EBM), that uses a modified diffusion equation to evaluate the meridional heat transport, with a radiative-convective public column model developed by NCAR. Using ESTM, we are able to evaluate the effect of observable astrophisical parameters such as the semi-major axis of the planet's orbit, the planet's radius and mass, the luminosity of the central star, and of less observable planetary parameters such as the inclination of the axis, the atmospheric pressure, the oceans' fraction, on the climate, and thus on habitability. An important characteristic of ESTM is that it is fast: each numerical computation takes minutes on a standard PC, and thus a detailed exploration of a large parameter space is viable. However, classical EBMs are known to be bistable: given a set of planetary parameters, it can happen that, depending on the initial temperature used to initialize the model, the final state is warm, with an average annual global temperature in the liquid water range, or frozen, a snowball state. Here we present preliminary results on the bistability of ESTM, that is not a classical model given its coupling with an atmospheric column model and other changes we did on the diffusion coefficient and on the estimate of ices and clouds (see Vladilo et al 2015 for details). We varied four parameters, namely semi-major orbital axis, surface pressure of the atmosphere, inclination of the rotation axis and orbit eccentricity. For each quadruplet of parameters, we used 29 different initial temperatures. In the (mono) stable cases, all computations do converge to the same annual global average temperature; in the bistable ones, we find snowball or warm planets depending on the initial temperature. We then identified and classified the (mono) stable and bi-stable cases, and studied the relations between stability, final states (warm or snowball) and habitability. We shortly discuss the implications of bistability on the habitability. 


\title{
ExoMars RSP Mission and the developed technologies for Planetary Protection
}

\author{
Musetti B. \& Margheritis D.B.
}

Corresponding author email: diana.margheritis@thalesaleniaspace.com

Keywords: Planetary Protection, Mars, procedures.

The ExoMars RSP Mission is a joint ESA-ROSCOSMOS mission including contributions from NASA, scheduled for launch in 2020 from the Baikonur Cosmodrome by a Proton-M/Breeze-M Launch Vehicle supplied by ROSCOSMOS. The spacecraft is made by an European Carrier Module, a Descent Module developed under Russian responsibility, carrying a Landing platform (LP) boarding a scientific suite of 12 instruments to study the Mars environment and housing the European Rover Module with its scientific Payload to search, after its egress from the LP, for present and past life signs on Mars. This mission is classified as COSPAR Planetary Protection Category IVb because is carring instruments to search for signs of past and present life on Mars. Planetary Protection analyses and procedures are implemented to compliant with COSPAR policy. The implementation of the ExoMars Mission 2020 Planetary Protection requirements is based on restrictions on impact probabilities, on Mars sample contamination and bioburden contamination control for parts of the spacecraft. Lessons learned from the ExoMars Mission 2016 are the foundation from which the ExoMars Mission 2020 Planetary Protection implementation is developed. But in the ExoMars Mission 2020 there is an additional layer of complexity: to avoid to contaminate the Mars sample with terrestrial contamination. A contamination controlled aseptic environment has been developed to allow the assembly and integration of the specific subsystem belong to the Mars sample pathway, in order to prevent any possible particulate, molecular or microbiological recontamination of the Mars sample. Specific procedures are developed to perform handling of the $\mathrm{H} / \mathrm{W}$ to the integration environment without breaking the sterility chain. The presentation provides a description and gives the current status on the implementation of the Planetary Protection activities in the frame of ExoMars Mission 2020 european side. 


\title{
Habitability of desert-like worlds: role of albedo, evapotranspiration and carbon feedbacks
}

\author{
Provenzale A. ${ }^{1}$, Lenzi S. ${ }^{1}$ \& Murante G. ${ }^{2}$ \\ ${ }^{1}$ Institute of Geosciences and Earth Resources, CNR, Pisa. \\ 2 Trieste Astronomical Observatory, INAF.
}

Corresponding author email: antonello.provenzale@cnr.it

Keywords: exoplanets, habitability, climate, modelling.

We discuss the habitability (in terms of the presence of liquid water) and the interaction between climate and vegetation on an ideal water-limited planet, focussing on the influence of vegetation on the planetary water cycle. We introduce a simple mechanistic box model with a two-layer representation of the atmosphere and a two-layer soil scheme. In this framework, we study the role of the albedo, evapotranspiration and carbon feedbacks and their effect on the potential planetary habitability. 


\section{On the challenge of quantifying the habitability of extrasolar planets}

Vladilo G. ${ }^{1}$, Silva L. ${ }^{1}$, Maris M. ${ }^{1}$, Murante G. ${ }^{1}$, Provenzale A. ${ }^{2}$, von Hardenberg J. ${ }^{3}$, Palazzi E. ${ }^{3}$, Simonetti P. ${ }^{4}$, Fulle M. ${ }^{1} \&$ Ivanovski S.L. ${ }^{1}$

${ }^{1}$ INAF - Osservatorio Astronomico di Trieste.

${ }^{2}$ IGG - CNR, Pisa.

${ }^{3}$ ISAC - CNR, Torino.

${ }^{4}$ Università di Trieste.

Corresponding author email: giovanni.vladilo@inaf.it

Keywords: exoplanets, atmosphere, biomarkers, modelling.

In the next few years we expect a rise of the number of terrestrial-type exoplanets that will be discovered with photometric techniques and monitored with radial-velocity measurements. The best targets to search for atmospheric biomarkers with the state-of-the art spectroscopic facilities will be selected among these planets. Since atmospheric spectroscopy of terrestrial-type planets is extremely demanding and time-consuming, the selection of the targets should be ideally driven by robust criteria of life sustainability. In this presentation we emphasize the challenges that must be faced to define quantitative criteria of habitability. Given the paucity of observational data, especially when compared with the situation typical of Solar System bodies, a large effort in the modelization of the climate and physico-chemical properties of the planetary surfaces and atmospheres is required. We show how climate models can be tailored for this type of task, taking into account the need to explore the parameter space that is not covered by observational data (Vladilo et al. 2015). With this approach we can provide a quantitative index of habitability that maximizes the probability of detecting atmospheric biomarkers (Silva et al. 2017). 


\section{PLANETS AND SATELLITES}




\title{
The Jupiter's Atmosphere from Juno
}

\author{
Adriani A. ${ }^{1}$, Mura A. ${ }^{1}$, Bolton S. ${ }^{2} \&$ the Juno team \\ ${ }^{1}$ INAF-Istituto di Astrofisica e Planetologia Spaziali, Roma, Italy. \\ ${ }^{2}$ Southwest Research Institute, San Antonio, Texas, USA.
}

Corresponding author email: albertoi.adriani@inaf.it

Keywords: Jupiter, Juno mission, Atmosphere, JunoCam, JIRAM.

Years of observations from Earth and other missions gave an image of the planet that was not complete. Since August 2016 Juno is operating at Jupiter observing its atmosphere. Juno represent an important milestone in the understanding the planet and its atmosphere. Jupiter's atmosphere is complex and turbulent. The poles were never observed like from Juno. The Juno visual camera, JunoCam, and the Jovian InfraRed Auroral Mapper, JIRAM, have revealed the presence of Earth-size cyclones densely clustered in different geometrical structures. Other atmospheric data come from the Juno's Microwave Radiometer (MWR), which samples the thermal microwave radiation from Jupiter's atmosphere, from the top of the ammonia clouds to hundred kilometers deep within its atmosphere. The data indicates that Jupiter's belt near the equator penetrates all the way down, while the belts and zones at other latitudes seem to evolve to other structures. The data suggest the ammonia concentration is quite variable and continues to increase as far down. MWR was also able to explain in depth the structure of the Great Red Spot. 


\title{
Temporal and Morphological Variability of Auroral Emissions from Juno/JIRAM data
}

\author{
Altieri F. ${ }^{1}$, Moriconi M.L. ${ }^{2}$, Dinelli B.M. ${ }^{3}$, Mura A. ${ }^{1}$, Adriani A. ${ }^{1}$, Migliorini A. ${ }^{1}$, Plainaki C. ${ }^{4}$, Grassi D. ${ }^{1}$, \\ Tosi F. ${ }^{1}$, Sindoni G. ${ }^{4}$, Piccioni G. ${ }^{1}$, Noschese R. ${ }^{1}$, Sordini R. ${ }^{1}$, Cicchetti A. ${ }^{1} \&$ the JIRAM team \\ ${ }^{1}$ IAPS-INAF, Via Fosso del Cavaliere 100, 00133 Roma. \\ 2 ISAC-CNR, Via Fosso del Cavaliere 100, 00133 Roma. \\ ${ }^{3}$ ISAC-CNR, via Gobetti, 101, Bologna, Italy. \\ ${ }^{4}$ Agenzia Spaziale Italiana, Rome, Italy.
}

Corresponding author email: francesca.altieri@inaf.it

Keywords: Jupiter, Juno mission, Jupiter aurorae, JIRAM.

The Jupiter InfraRed Auroral Mapper (JIRAM) instrument on board the NASA Juno mission is an imaging spectrometer designed to study both the atmosphere and auroral features of the planet. In the 3-4-micron spectral range JIRAM spectra clearly show the emissions of the H3+ ion and of methane. In this study we compare the observations collected in August 2016 with those performed in early February 2017 on both the northern and southern polar regions to investigate variations in intensity and morphology of the auroral features. We also study the background emissions outside the main oval toward the equator. We find that the auroral emissions are highest in August 2016 on the southern aurora, while an increase of the $\mathrm{CH} 4$ emission after noon is observed in the polar region inside the southern main oval during February 2017, when this area has been fully covered by JIRAM data. Temporal and morphological variations are correlated with the UVIS observations and Solar wind dynamic pressure and discussed. 


\title{
Karst landforms within Cagli crater, Mars as useful lithological and paleo-climatic markers
}

\author{
Baioni D.
}

Dipartimento di Scienze Pure e Applicate (DISPeA) Università degli studi di Urbino Carlo Bo, Campus Scientifico “E. Mattei” 61029 Urbino (PU) Italia.

Corresponding author email: davide.baioni@uniurb.it

Keywords: Mars, MRO, HiRISE, Light toned deposits, Karst.

This work describes the karst landforms observed in the Light Toned Deposits (LTDs) within Cagli crater, a medium size crater located in northern Sinus Meridiani, an area near to the equatorial region of Mars. A detailed morphological and morphometric survey of the LTDs surface morphologies was carried out through an integrated visual analysis of the available Reconnaissance Mars Orbiter (MRO) High Resolution Imaging Science Experiment (HiRISE) and the Context Camera (CTX) images. The Martian landforms interpreted as karst sinkholes after discarding the other possible origins, resemble strongly karst landforms that can be observed both in different karst terrains on the Earth and in other regions of Mars. The karst landforms observed suggest a young erosional age, highlight the evaporitic origin of these LTDs and seem to confirm the presence of liquid water, probably due to ice melting, during the late Amazonian age. 


\section{The NOMAD experiment on board TGO/Exomars 2016: First results}

Bellucci G. ${ }^{1}$, Vandaele A.C. ${ }^{2}$, Lopez-Moreno J.-J. ${ }^{3}$, Patel M.R. ${ }^{4}$, Daerden F. ${ }^{2}$, Depiesse C. ${ }^{2}$, Mason J., Ristic B. ${ }^{2}$, Thomas I.R. ${ }^{2}$, Willame Y. ${ }^{2}$, Oliva F. ${ }^{1}$, D’Aversa E. ${ }^{1}$, Altieri F. ${ }^{1}$ \& Carrozzo F.G. ${ }^{1}$

${ }^{1}$ INAF-IAPS, Rome, Italy.

${ }^{2}$ IASB-BIRA, Bruxelles, Belgium.

${ }^{3}$ IAA-CSIC, Granada, Spain.

${ }^{4}$ The Open University, Milton Keynes, United Kingdom.

Corresponding author email: giancarlo.bellucci@inaf.it

Keywords: Martian atmosphere, Exomars, TGO, Spectroscopy, NOMAD.

The NOMAD (Nadir and Occultation measurements for Mars Discovery) spectrometer suite onboard the joint ESA-Roscosmos ExoMars Trace Gas Orbiter mission is mapping the composition and distribution of Mars' atmospheric trace species in unprecedented detail. The instrument is a combination of three channels, covering a spectral range from the UV to the IR, and can perform solar occultation, nadir and limb observations. NOMAD is an international collaboration led by Belgium, with major contributions from Italy, Spain and UK. Following the Mars Express experience, part of the Italian community is involved on this new exciting mission. NOMAD has the capability to study the abundance of trace gases in the Martian atmosphere, with attention to methane due to its astrobiological implications. In this talk the NOMAD scientific objectives and first results will be reviewed. 


\title{
Geomorphological mapping of Cilaos - Gasa impact crater complex
}

\author{
Bertoli S. ${ }^{1}$, Pacifici A. ${ }^{2}$, Baroni C. ${ }^{1,3} \&$ Salvatore M.C. ${ }^{1,3}$ \\ ${ }^{1}$ Dipartimento di Scienze della Terra, Università di Pisa, Italy. \\ ${ }^{2}$ International Research School of Planetary Sciences, Viale Pindaro, 42, Pescara, Italy. \\ ${ }^{3}$ CNR- IGG, Consiglio Nazionale delle Ricerche - Istituto di Geoscienze e Georisorse, Pisa, Italy.
}

Corresponding author email: silvia.vickybertoli@gmail.com

Keywords: Mars, Impact crater, Cilaos-Gasa crater.

Gasa Crater $\left(35.72^{\circ} \mathrm{S}, 129.45^{\circ} \mathrm{E}\right)$ is a fresh $7-\mathrm{km}$ diameter impact crater on Mars, located within an older $18-\mathrm{km}$ diameter crater, Cilaos, in eastern Promethei Terra. The Gasa impact event is localized in an age range of 0.6-2.4 Ma [1], making it one of the youngest kilometre-scale craters on Mars. It is characterized by a sharp rim crest, with a multitude of engraved gullies on the pole-facing inner wall, that show well-defined alcovechannel-apron morphologies [2]. According to [3], some of these gullies displays recent activities. The crater floor is flat with a diffusive terrestrial-like polygon karst features and bedrock outcrops. Cilaos instead, is degraded with evidence of latitude dependent-mantling [4], gullies and periglacial-like morphologies. These peculiarities (first of all, the young age of Gasa) make this area very interesting in order to investigate the last period of Amazonian era (3 Ga-present). This work is focused on the mapping and 3D characterization of different features occurring in both craters, to better understand the answer of Martian surface to the climate change thought the last few million years.

\section{References:}

[1] Schon et al. (2009) - Unique chronostratigraphic marker in depositional fan stratigraphy on Mars: Evidence for ca. 1.25 Ma gully activity and surficial meltwater origin. Geology.

[2] Okubo et al. (2011) - Constraints on mechanisms for the growth of gully alcoves in Gasa crater, Mars, from twodimensional stability assessments of rock slopes. Icarus.

[3] Dundas et al. (2010) - New and recent gully activity on Mars as seen by HiRISE. Geophysical Research Letters.

[4] Schon \& Head (2012) - Gasa impact crater, Mars: Very young gullies formed from impact into latitude-dependent mantle and debris-covered glacier deposits? Icarus. 


\title{
Geometrical properties of landslides on Earth and Mars
}

\author{
Brunetti M.T. ${ }^{1}$, Ardizzone F. ${ }^{1}$, Peruccacci S. ${ }^{1} \&$ Guzzetti F. ${ }^{1}$ \\ ${ }^{1}$ Istituto di Ricerca per la Protezione Idrogeologica del CNR, Perugia.
}

Corresponding author email: mariateresa.brunetti@irpi.cnr.it

Keywords: landslides, Earth, Mars, Valles Marineris.

On Earth, landslides occur in various morphological and climatic settings and can strongly affect the evolution of a landscape. On Mars, slope failures have been also observed since decades (e.g., [1], [2]), especially in the large canyons of Valles Marineris. In the perspective of a novel interest in the human settlement on the red planet it is important to explore the local environmental conditions and the possible hazards. For the purpose, we analyzed the geometrical features of landslides on Earth and Mars and compared the relationships between their geometrical parameters (i.e. landslide volumes, areas, drop heights and runout distances). Data for this study were collected using landslide catalogues available from several authors $[3,4,5,6,7]$. In particular, we selected rock and debris slides, rock avalanches on Earth and Mars and terrestrial submarine landslides. Having insights on the geometrical properties of the landslides on the two planets might provide tools for the understanding of the sliding mechanisms in different environments.

\section{References:}

[1] Lucchitta (1979) - Journal of Geophysical Research: Solid Earth, 84(B14), 8097-8113.

[2] McEwen (1989) - Geology, 17(12), 1111-1114.

[3] Guzzetti et al. (2009) - Earth and Planetary Science Letters, 279(3-4), 222-229.

[4] Strom and Abdrakhmatov (2018) - Elsevier, 459 pp., ISBN: 9780128032046.

[5] Quantin et al. (2004) - Planetary and Space Science, 52(11), 1011-1022.

[6] Brunetti et al. (2014) - Earth and Planetary Science Letters, 405, 156-168.

[7] Lucas et al. (2014) - Nature Communications, 5, 3417. 


\title{
Identification and characterization of possible Martian landing sites
}

\author{
Camplone V. ${ }^{1}$, Sindoni G. ${ }^{1}$, Negri B. ${ }^{1}$, Ammannito. E. ${ }^{1}$, Altieri. F. ${ }^{2}$, Carrozzo F.G. ${ }^{2}$, Cremonese G. ${ }^{3}$, \\ Pajola M. ${ }^{3}$, Pirrotta S. ${ }^{1} \&$ Zinzi A. ${ }^{4}$ \\ ${ }^{1}$ Italian Space Agency (ASI). \\ ${ }^{2}$ Institute for Space Astrophysics and Planetology (IAPS-INAF). \\ ${ }^{3}$ Astronomical Observatory of Padova (OAPd-INAF). \\ ${ }^{4}$ Space Science Data Center - ASI (SSDC-ASI).
}

Corresponding author email: veronica.camplone@est.asi.it

Keywords: Mars, Landing site, Martian surface.

In the past few years, the growing interest towards the exploration of Mars required the development of new missions that call for in-situ analysis of soil samples through the usage of landers and rovers. The selection of appropriate landing sites must abide to precise both scientific and technical/engineering requirements. The aim of this study is to provide useful bases for the investigation and identification of areas on the Martian surface that exhibits geological hints of past wet climatic periods [1]. We carried out the identification of such areas through a geological analysis. By means of both infrared and visible cameras, it was possible to search for the occurrence of fluvial/glacial erosion, primary markers of past habitability. To carry out the geological characterization, we employed CTX/MRO [2] visible, and THEMIS/MO [3] visible/infrared cameras. We performed an evaluation of the obtained images through the QGIS (Geographic Information System) open source software, with which we were able to carry out various types of geomorphological (e.g. slopes, asperity index, etc), morphometric and topographical analysis. This allowed us to draw a cartography of the areas considered geologically interesting. Then, we will perform a mineralogical characterization of the areas previously selected, by means of infrared spectra from CRISM/MRO [4] and OMEGA/MEX [5] instruments. Finally, we will deepen the analysis using the latest CaSSIS/TGO [6] images and DTM.This study is important for the scientific community since it could be considered a reference for the planning of future cameras and spectrometers observations, and could identify possible areas of interest for the upcoming missions to Mars.

\section{References:}

[1] Cabron et al. (2001) - ICARUS.

[2] Malin et al. (2006) - JGR.

[3] Christensen et al. (2001) - SSR.

[4] Murchie et al. (2007) - JGR.

[5] Bibring et al. (2004) - ESA.

[6] Thomas et al. (2016) - SSR. 


\title{
Proclus Crater: mapping the spectral variability within Lunar Highlands
}

\author{
Carli C. ${ }^{1}$, Serventi G. ${ }^{2}$, Giacomini L. ${ }^{1}$, Pozzobon R. ${ }^{3}$ \& Sgavetti M. ${ }^{2}$ \\ ${ }^{1}$ IAPS - INAF. \\ ${ }^{2}$ Dipartimento di SCVSA - Univeristà di Parma. \\ ${ }^{3}$ Dipartimento di Geoscienze - Università di Padova.
}

Corresponding author email: cristian.carli@inaf.it

Keywords: Moon, Proclus crater, Lunar highlands.

Mafic regions embedded in the Lunar highlands have been recently observed [1] and, moreover, the presence of an absorption band at ca. $1250 \mathrm{~nm}$, assigned to Fe2+ transition in PL, has been detected in central peaks of some impact craters (e.g. [2]). Here, we analyze M3 (Chandrayaan mission) reflectance spectra of Proclus crater, a $28 \mathrm{~km}$ crater located in a highland portion on the west of Mare Crisium, were high amount of PL have been previously detected (e.g. [3]). First, we classified the crater in different spectral regions, using the Spectral Angle Mapper method, with a spectral library build by means of Purity Pixel Index, on the basis of the different spectral properties in the reflectance data. Then, we related the spectral parameters of each region to the mineralogical composition. To this purpose, the M3 data have been compared with laboratory spectra acquired on well characterized mixtures of PL and mafic minerals, such as pyroxene (PX) and olivine (OL) (e.g. [4] and references therein). We recognized regions with different spectral behaviors: 1) PL regions located mainly in the crater walls; 2) PX regions in both the walls and the floor of the crater; 3) OL regions in the southeast portion of the crater walls; and 4) PL ( $90 \%)+$ PX regions in the crater wall. Here we have highlighted how a comparison with laboratory spectra can strongly help to obtain information about the mineral content and composition of a planetary surface. Moreover, a comparison with morphology and a 3D reconstruction is ongoing to assess a geological setting to this fresh crater and identify the origin of these mafic outcrops.

This work was partially supported by European Union's Horizon 2020 research grant agreement No 776276- PLANMAP.

\section{References:}

[1] Yamamoto et al. (2015) - J.G.R. Planets, 120.

[2] Cheek L.C. et al. (2012) - 2nd conference on the lunar highland crust. Ab\# 9022.

[3] Donaldson Hanna et al. (2014) - J.G.R. Planets, 119.

[4] Serventi et al. (2016) - Icarus, 272. 


\section{SIMBIO-SYS present and future}

Cremonese G. ${ }^{1}$, Capaccioni F. ${ }^{2}$, Capria M.T. ${ }^{2}$, Palumbo P. ${ }^{3}$, Debei S. ${ }^{4}$, Bettanini C. ${ }^{4}$, Cicchetti A. ${ }^{2}$, Da Deppo V. ${ }^{5}$, De Sanctis M.C. ${ }^{2}$, Filacchione G.2, Massironi M. ${ }^{6}$, Noschese R. ${ }^{2}$, Politi R. ${ }^{2}$, Re C. ${ }^{1}$, Simioni E. ${ }^{1}$, Slemer A. ${ }^{5} \&$ Zusi M. ${ }^{2}$

${ }^{1}$ INAF-Astronomical Observatory of Padova, Italy.

${ }^{2}$ INAF-IAPS Roma, Istituto di Astrofisica e Planetologia Spaziali di Roma, Italy.

${ }^{3}$ Dipartimento di Scienze e Tecnologie, Università di Napoli "Parthenope".

${ }^{4}$ CISAS, University of Padova, Italy.

${ }^{5}$ IFN, CNR, Padova, Italy.

${ }^{6}$ Department Geosciences, University of Padova, Italy.

Corresponding author email: gabriele.cremonese@oapd.inaf.it

Keywords: Mercury, BepiColombo, SIMBIO-SYS.

SIMBIO-SYS is the suite of three remote sensing instruments on board the ESA-JAXA mission BepiColombo, that will provide high resolution images (HRIC), the 3D (STC) and spectral (VIHI) global mapping of Mercury. The design of SIMBIO-SYS is enriched of new technologies that allowed to realize a really compact instrument, having a total mass of $14.5 \mathrm{~kg}$. The Near Earth Commissioning has been successfully completed, but we have to wait for 7 years before getting the first data on Mercury. In the meantime, we have to prepare the observing strategy, according to revised scientific objectives taking into account the MESSENGER observations, and the target list. The innovative design of SIMBIO-SYS makes it small and light, but allowing good performances that may be challenging even for the exploration of other bodies. Recently we have started to think about having SIMBIO-SYS on board a spacecraft around the Moon, applying minor changes to the design it is possible to collect unprecedented data, as for instance searching for water on the polar regions. We will describe the present performances of the instrument and how they can be interesting even for the Moon. 


\title{
Investigation of superficial features of the Galilean moons by 3GM experiment aboard ESA JUICE mission
}

\author{
De Marchi F. ${ }^{1}$, Di Achille G. ${ }^{2}$, Di Benedetto M. ${ }^{1}$, Di Stefano I. ${ }^{1} \&$ Cappuccio P. ${ }^{1}$ \\ ${ }^{1}$ Department of Mechanical and Aerospace Engineering, Sapienza University of Rome, Italy. \\ ${ }^{2}$ INAF - National Institute for Astrophysics - Astronomical Observatory of Abruzzo, Italy.
}

Corresponding author email: fabrizio.demarchi@uniroma1.it

Keywords: JUICE mission, Galileian satellites, Gravity field, Interior structure.

The Geodesy and Geophysics of Jupiter and the Galilean Moons (3GM) experiment aboard the JUpiter ICy moons Explorer (JUICE) will estimate the gravity field of Europa, Callisto (5x5) and Ganymede (40x40). The information about the gravity field can be used to characterize the interior structure of the satellite as well as to identify possible gravity anomalies related to mass concentrations generated by tectonic structures and/or non-homogeneous density distribution within the ice shell. The quadrupole term for Callisto and Ganymede have been set from the Galileo mission; [1,2] for Callisto the higher order terms have been simulated using the Kaula's rule [3]. With 3GM it will be possible to detect large (thousands of km) surface structures for Callisto, since the data will be acquired only during twelve flybys. While the large amount of data retrieved from the orbital phase will let us to achieve enough detail to potentially identify regional (hundreds of $\mathrm{km}$ ) structures for Ganymede. Remarkably, the Ganymede's outer ice shell is characterized by the presence of dark and bright terrains: evidence of older and dirty and younger and clean ice, respectively. We implemented a 3GM simulation to investigate under which conditions (in terms of ice depth and density contrast) it could be possible to detect putative gravity anomalies reflecting the surface distribution of dark and bright terrains. The Ganymede's gravity field has been generated up to the degree 50, based on the observed distribution of the dark and bright terrains and assuming a density contrast of $50 \mathrm{~kg} / \mathrm{m}^{3}$ between the two types of ice and an ice depth of $25 \mathrm{~km}$. With these assumptions, our preliminary results show that $3 \mathrm{GM}$ can potentially identify gravity anomalies due to the presence of dark and bright terrains.

\section{References:}

[1] Anderson et al. (2001) - Icarus, 153, 1, 157-161.

[2] Anderson et al. (1996) - Nature, 384, 541-543.

[3] Bertotti et al. (2003) - Physics of the Solar System, Springer Science+Business-Media. 


\title{
Martian fan-deltas and the $\Delta \mathrm{H} 2 \mathrm{O}$ throughout the geological history of Mars: insights from sedimentary mass balance
}

\author{
Di Achille G. ${ }^{1} \&$ Vaz D.A. ${ }^{1,2}$ \\ ${ }^{1}$ Istituto Nazionale di Astrofisica - Osservatorio Astronomico d'Abruzzo, Italy. \\ ${ }^{2}$ Centre for Earth and Space Research of the University of Coimbra, Portugal.
}

Corresponding author email: gaetano.diachille@,inaf.it

Keywords: Mars, Vallyes, Fan-shaped deposits.

Using high-resolution topography, we present a detailed mass balance analysis of Martian valleys and fan-shaped deposits, providing new insights into past depositional environments. Based on morphometric and volumetric measurements, we found that two main types of Martian fans can be discriminated. Type I fans are more numerous $(\sim 70 \%)$, relatively well preserved, associated with smaller and less mature drainage networks and were deposited on stepper gradients. The balance between eroded and deposited materials indicates that these fans might have been formed by depositional processes other than typical deltaic sedimentation, since a significant loss of sediments during deposition did not occur (volume ratio of eroded vs. deposited materials of approximately 1). We propose that the identified type I fans formed by a prevalence of gravity-driven flows not necessarily deposited on subaqueous settings. In contrast, type II fans are less abundant, highly eroded, were deposited on flatter areas and formed downstream of more mature drainage networks with longer, deeper and wider valleys. The mass balance for this set of fans shows that considerable amounts of sediment were not retained in the fans, implying a large offshore bypass of sediments during their formation (volume ratios between 3 and 10). These evidences support a fluvial-deltaic origin for this class of fans, indicating the existence of paleolakes during substantial periods of time. Our results suggest that only a small percentage of the fans $(\sim 30 \%)$ are consistent with the occurrence of favorable and durable conditions for life, i.e. long-lived integrated fluvial, deltaic and lacustrine environments. Whereas, the majority of Martian fan-shaped deposits might have been formed with significant contributions of alternative processes (gravity-driven) besides fluvial transport and deposition also by episodic events that do not necessarily imply the occurrence of a global and clement climate during their formation. 


\title{
Geomorphology and Sedimentology of southwestern Lake General Carrera/Buenos Aires (Chile/Argentina): Implications for Martian fluvio-lacustrine deposits
}

\author{
Di Achille G. ${ }^{1}$, Salese F. ${ }^{2} \&$ Vaz D.A. ${ }^{1,3}$ \\ ${ }^{1}$ Istituto Nazionale di Astrofisica - Osservatorio Astronomico d'Abruzzo, Italy. \\ ${ }^{2}$ Faculty of Geosciences, Utrecht University, Netherlands, 3 Centre for Earth and Space Research of the University of \\ Coimbra, Portugal.
}

Corresponding author email: gaetano.diachille@,inaf.it

Keywords: Lake General Carrera/Buenos Aires, deglaciation, Martian lacustrine systems.

Due to climate change and the last major deglaciation of the Northern Patagonian Icefield, Lake General Carrera/Buenos Aires (LGC/BA), experienced significant hydrological modifications, which resulted in a more than $400 \mathrm{~m}$ vertical retreat of the lake through five main stages during the last $15 \mathrm{k}$ years. The paleohydrology of the LGC/BA is investigated as well as the geomorphology and sedimentology of the fan-delta deposits and associated coastal features. Reconstructing the retreat of the LGC/BA elucidates the morphodynamics, formation and erosion times of the numerous deltaic deposits formed along the shores of the LGC/BA during the fast and progressive melting helps to gain a better understanding of sedimentary processes during rather fast and compelling climatic, hydrological, and water base level changes. The latter conditions might have been responsible for the formation of short-lived Martian lacustrine systems under climatic conditions not necessarily different from those of present Mars. For this reason, LGC/BA is a unique terrestrial analog to test fluvio-deltaic sedimentary catastrophic scenarios characterized by a) relatively high water and sediment discharges (due to glacial retreat and ice-melt) and b) rapid and significant water level changes (due to the high water influx and break of ice dams and fast draining of the lake). LGC/BA provides access to a concentrate of present and past coastal lacustrine morphology, erosional and depositional features, which can be directly compared with Martian putative fluvio-lacustrine deposits and associated features. Moreover, the qualiquantative study of fan-deltas at LGC/BA has strong implications for several open issues related to Martian fan-deltas. We present results from two fieldwork campaigns at LGC/BA and remote sensing analysis using all the available datasets and discuss the implications for the hydrological and climatic significance of Martian deltaic deposits. 


\title{
The 3GM experiment on board the ESA JUICE mission
}

\author{
Di Benedetto M. ${ }^{1}$, Cappuccio P. ${ }^{1}$, Ciarcia S. ${ }^{2}$, Iess L. ${ }^{1}$, Kaspi Y. ${ }^{3}$ \& Mann R. ${ }^{4}$ \\ ${ }^{1}$ Dipartimento di Ingegneria Meccanica e Aerospaziale, Sapienza università di Roma. \\ 2 Thales Alenia Space - Italy. \\ ${ }^{3}$ Weizmann Institute of Science, Department of Earth and Planetary Science. \\ ${ }^{4}$ AccuBeat Ltd.
}

Corresponding author email: mauro.dibenedetto@uniroma1.it

Keywords: JUICE, gravity, geophysics, Jovian system.

JUICE is an ESA L-class mission devoted to study the three moons Europa, Ganymede and Callisto and the Jovian system in all its complexity. The spacecraft will be launched in mid-2022 and it will reach Jupiter in 2030, starting the 3-year nominal tour that will end with a 4-month circular orbital phase around Ganymede, the main target of the mission. JUICE will host ten different scientific instruments, broadly divided into a remote sensing, a geophysical and an in situ package. The 3GM (Gravity and Geophysics of Jupiter and the Galilean Moons) radio science instrument consists of two different and separated units, namely a Ka band Transponder (KaT) and an Ultra Stable Oscillator (USO), that will address scientific objectives pertaining to gravity, geodesy and geophysics as well as atmospheric science with radio occultations. The main goal of the gravity experiments is the determination of the moons' gravity fields and, for Ganymede and Callisto, the unambiguous detection of the internal oceans. The KaT is the core of the gravity experiment, enabling a highly stable two-way coherent link at Ka band (34.5-32.2 GHz). It is based on a fully digital architecture, allowing an intrinsic Allan deviation (ADEV) lower than $10^{\wedge}-15$ at $1000 \mathrm{~s}$ integration time. It also supports a Pseudo Noise 24.35 Mcps modulation scheme, enabling range measurements as accurate as $20 \mathrm{~cm}$ (two-way) that will be used to improve the ephemerides of the Jovian system and to carry out tests of GR. The USO will provide a stable reference signal (ADEV at level of few $10^{\wedge}-13$ at 1-1000 s integration times) for one-way downlink measurements at $\mathrm{X}$ and $\mathrm{Ka}$ band. The radio signal generated from the USO will pass through (occulted by) Jupiter and icy moons atmospheres, allowing the retrieval of horizontal and vertical profiles of pressure and temperature, variability of electron densities, atmospheric wave characteristics and the magnetosphericionosphere coupling processes on Jupiter. 


\title{
Determination of the Mercury's Moho depth with ESA BepiColombo MORE
}

\author{
Di Stefano I. ${ }^{1}$, Cappuccio P. ${ }^{1}$, De Marchi F. ${ }^{1} \&$ D’Achille G. ${ }^{2}$ \\ ${ }^{1}$ Dipartimento di Ingegneria Meccanica e Aerospaziale - Università di Roma "La Sapienza”, via Eudossiana 18, 00184 \\ Rome, Italy. \\ ${ }^{2}$ Istituto Nazionale di Astrofisica (INAF), Osservatorio Astronomico di Teramo, Viale M. Maggini, Teramo, Italy 0000- \\ 0002-2151-4057.
}

Corresponding author email: ivan.distefano@uniroma1.it

Keywords: Mercury, BepiColombo, MESSENGER, Moho.

Aim of this work is to combine gravity field and topographical data to obtain a map of the Mercury's crust thickness and its uncertainty. The Bouguer anomalies are thus used to determine the spherical harmonic coefficients, which describe the crust-mantle density interface. From this information along with topographical data, it is possible to retrieve the crustal thickness. Our results show how the combination of BepiColombo high precision gravity measurements and topographic information can lead to the determination of a Mercury's crust thickness map with realistic uncertainties.

For our implementation we used the values of Mercury 's crust density $(2974 \mathrm{~kg} / \mathrm{m} 3)$ and the average crust thickness $(26 \mathrm{~km})$ from [1], and of mantle density $(3200 \mathrm{~kg} / \mathrm{m} 3)$ from [2], respectively.

We generated 1000 gravity fields perturbing the HgM007 of MESSENGER3 based on the covariance matrix obtained by simulation of BepiColombo Mercury Orbiter Radioscience Experiment (MORE). As far as the topographic data, we used the MESSENGER GTMES_150v05.4

For each generated gravity field, we adopted the method explained in [2] to obtain the Moho interface and consequently the 1000 maps of crust's thickness. For each point of the map we calculated the mean and the standard deviation, obtaining a map of the mean value and the uncertainty of the crust thickness. The uncertainty resulted from the simulations allow us to confidentially correlate the gravity anomalies and crustal thickness maps with surface morphotectonic structures (such as impact craters, rifts, lobate scarps etc.).

\section{References:}

[1] Sori (2018) - Earth and Planetary Science Letters, 489,

[2] Wieczorek et al. (2015) - Gravity and Topography of the Terrestrial Planets, University Paris Diderot, Paris Cedex 13, France.

[3] http://pds-geosciences.wustl.edu/messenger/mess-h-rss_mla-5-sdp-v1/messrs_1001/data/shbdr/

[4] http://pds-geosciences.wustl.edu/messenger/mess-h-rss_mla-5-sdp-v1/messrs_1001/data/shadr/ 


\title{
A peek inside the gas giants with gravity measurements
}

\author{
Durante D. ${ }^{1}$, Notaro V. ${ }^{1}$, Racioppa P. ${ }^{1} \&$ Iess L. ${ }^{1}$ \\ ${ }^{1}$ Sapienza Università di Roma.
}

Corresponding author e-mail: daniele.durante@uniroma1.it

Keywords: Jupiter, Saturn, Cassini mission, Gravity field, Interior structure.

Recently, the Juno and Cassini spacecraft shed light on the interior of both Jupiter and Saturn, the two gas giants of the Solar System. The Juno spacecraft is currently orbiting Jupiter in a highly eccentric, 53.5-day orbit, with a perijove altitude of about $4000 \mathrm{~km}$. Since its arrival in Jupiter's system on July 4th, 2016, Juno has completed several revolutions about the planet, revealing fundamental details of Jupiter's interior structure and atmospheric dynamics. On the contrary, the Cassini spacecraft ended its mission on September 15th, 2017 with a deliberate plunge into Saturn's atmosphere. In its final phase, the Grand Finale, Cassini provided insights on Saturn's rings, atmosphere, and interior. Out of the 22 proximal orbits, six pericenter passes have been devoted to the determination of the gravity field of the planet. Juno's first two passes have shed light on the interior by suggesting the presence of a diluted core. The analysis of the first two gravity-dedicated perijove passes (PJ03 and PJ06) revealed the North-South asymmetry in Jupiter's gravity field, which have been explained by the surface wind dynamics. The comparison of the observed even and odd zonal harmonics with theoretical predictions revealed that the surface zonal winds extend to a depth of thousands of kilometers. Cassini's gravity dedicated passes provided the most accurate determination of Saturn's gravity field since the spacecraft passed very close to the cloud tops. The analysis of the data acquired by Cassini revealed that Saturn's gravity deviates significantly from earlier theoretical expectations of a uniformly rotating body and requires a strong differential rotation extending deep into the planet. Juno and Cassini determined Jupiter's and Saturn's gravity field almost at the same time, enabling comparisons between the two gas giants of our solar system. We will explore the implications of Juno's and Cassini's gravity measurements on the interior of their hosting planet. 


\title{
Detection, classification and mapping of rilles in the northern hemisphere of the Moon
}

\author{
Fiorucci S. ${ }^{1}$, Podda S. ${ }^{2}$, Collu C. ${ }^{2}$, Brunetti M.T. ${ }^{3}$, Melis M.T. ${ }^{2} \&$ Scaioni M. ${ }^{4}$ \\ ${ }^{1}$ Dipartimento di Fisica e Geologia, Università di Perugia. \\ 2 Dipartimento di Chimica e Scienze Geologiche, Università di Cagliari. \\ ${ }^{3}$ Istituto di Ricerca per la Protezione Idrogeologica del CNR, Perugia. \\ ${ }^{4}$ Dipartimento di Architettura, Politecnico di Milano.
}

Corresponding author email: mariateresa.brunetti@irpi.cnr.it

Keywords: rilles, Moon, classification, morphometric features.

Lunar rilles are any of the long, narrow depressions on the surface of the Moon. These structures have different characteristics, which are related to their origin. Here, a classification of the lunar rilles in different groups with similar morphometric features and likely the same formation mechanisms is proposed. The detection, classification and mapping of lunar rilles has been achieved in two quadrants of the northern hemisphere, which are located between $-90^{\circ} \mathrm{E}$ to $90^{\circ} \mathrm{E}$ longitude and $0^{\circ} \mathrm{N}$ to $60^{\circ} \mathrm{N}$ latitude. For the detection and mapping of rilles, we used images from the Lunar Reconnaissance Orbiter Camera (LROC) [1] which have a resolution of $100 \mathrm{~m} /$ pixel and the DEM from Lunar Orbiter Laser Altimeter (LOLA) [2] with a resolution of $6 \mathrm{~m} / \mathrm{pixel}$.

Lunar rilles include generic sinuous rilles (SR), cracks on lava deposits (CL), surface lava flows (LF), subsurface lava tubes or catena (crater chains, CC) and tectonic structures (TS). For each type of the classified structures, morphologic parameters, i.e. length, width, perimeter, area, depth, longitude and latitude have been measured in order to allow a better understanding of how these features formed [3]. For those sinuous rilles classified as remnants of shallow lava channels or subsurface collapsed lava tubes, morphometric analysis included the extraction of the sinuosity index and of the regional slope and azimuth.

This work is born inside an international cooperation between Italy and China called "Moon Mapping project" for the cooperation between Italian and Chinese college students [4].

\section{References:}

[1] Chin et al. (2007) - Space Sci. Rev. 129, 391-419.

[2] Smith et al. (2010) - Space Sci. Rev. 150, 209-241.

[3] Hurwitz et al. (2013) - Planet. and Space Sci. 79-80, 1-38.

[4] Scaioni et al. (2016) - Int. Arch. Photogramm. Remote Sens. Spatial Inf. Sci., XLI-B6, 71-78. 


\title{
Regional-scale study of the structural asset of the high-Mg region of Mercury
}

\author{
Galluzzi V. ${ }^{1}$, Giacomini L. ${ }^{1}$, Guzzetta L. ${ }^{1}$, Ferranti L. ${ }^{2}$, Massironi M. ${ }^{3} \&$ Palumbo P. ${ }^{1,4}$ \\ ${ }^{1}$ INAF, Istituto di Astrofisica e Planetologia Spaziali, Rome, Italy. \\ ${ }^{2}$ Dipartimento di Geoscienze, Università degli Studi di Padova, Padua, Italy. \\ ${ }^{3}$ DISTAR, Università degli Studi di Napoli "Federico II", Naples, Italy. \\ ${ }^{4}$ Dipartimento di Scienze \& Tecnologie, Università degli Studi di Napoli “Parthenope”, Naples, Italy.
}

Corresponding author email: valentina.galluzzi@inaf.it

Keywords: Mercury, High-Mg region, Geochemistry, Tectonic patterns.

The High-Mg region (HMR) is a large area of Mercury $\left(30-150^{\circ} \mathrm{W} ; 25^{\circ} \mathrm{S}-60^{\circ} \mathrm{N}\right)$, where the highest $\mathrm{Mg} / \mathrm{Si}$ ratio is observed (mostly_ 0.5 ) [1], and where crustal densities should be higher with respect to the surrounding areas [2]. Its origin is ascribed to lateral heterogeneities in a poorly mixed mantle [3]. A structural analysis performed on the geological map of the Victoria quadrangle [4] highlights that the geochemical variations well correspond to a structural dichotomy due to the spatial distribution of non-parallel fault systems: a N-S fault system (VS) to the east, versus a NE-SW fault system (LS) to the west. Specifically, whereas LS is mostly found within the HMR, VS form the eastern and the northeastern boundary of the region. The spatial correlation between the geochemistry [1,2] and the regional-scale tectonic patterns leads to important insights on the evolution, orientation and kinematics of faults. We suggest that the HMR worked as a dense "plate", where global contraction stresses concentrated mostly at its borders. The observable structural and compositional relationships evolved in concert, playing a major role in shaping this area of Mercury. The faults may have followed previous HMR discontinuities and, in turn, may have deformed the HMR itself. In order to verify these relationships elsewhere, and find out the exact tectonic evolution of this wide region, we will compare the structural asset of other quadrangles where geological mapping is ongoing [5], and which are encompassed within, or at the borders of the HMR.

We gratefully acknowledge funding from the Italian Space Agency (ASI) under ASI-INAF agreement 2017-47-H.0.

\section{References:}

[1] Weider et al. (2015) - Earth Planet Sci Letters.

[2] Namur \& Charlier (2017) - Nat. Geosci.

[3] Frank et al. (2017) - J Geophys Res Planets.

[4] Galluzzi et al. (2016) - J Maps.

[5] Galluzzi et al. (2018) - In: Mercury, LPI Cont. 


\title{
Mercury's Crust and Core from MESSENGER Geodetic Investigation
}

\author{
Genova A. \\ Università di Roma La Sapienza.
}

Corresponding author email: antonio.genova@uniroma1.it

Keywords: Mercury, MESSENGER, Radio science, Gravity anomalies, Crustal thickness.

The MErcury Surface, Space ENvironment, GEochemistry, and Ranging (MESSENGER) spacecraft orbited Mercury for more than 4 years, from March 2011 to April 2015. It was equipped with onboard radio frequency (RF) telecommunication subsystem that enabled precise range and range rate observations for radio science. MESSENGER mission addressed key scientific objectives focused on the interior of the planet with the radio science investigation. The analysis of the entire MESSENGER radio science dataset includes data from the three Mercury flybys (2008 and 2009) and the full orbital mission (from March 2011 to April 2015). The first years of radio data allowed us to determine the gravity field of Mercury with a resolution of $\sim 150 \mathrm{~km}$ in the northern hemisphere since MESSENGER pericenter was located at higher latitudes $\left(665^{\circ} \mathrm{N}\right)$ and 200-500 km altitudes. The last year of the MESSENGER extended mission was dedicated to a low-altitude campaign, where the spacecraft pericenter was maintained at altitudes between 15 and $100 \mathrm{~km}$. The radio data collected during this mission phase enabled a significant improvement of the resolution of the gravity field. We present the gravity anomalies and crustal thickness maps that lead to a better understanding on the formation and evolution of specific regions. Our new gravity solution also provides refined estimates of the spin axis coordinates (right ascension and declination) that permits the retrieval, for the first time, of the average obliquity of the whole planet, which we find in perfect agreement with the Cassini state. The normalized polar moment of inertia, $\mathrm{C} /$ MR2, resulting from the new obliquity is significantly lower than the previous estimate. Our new measurement of the polar moment of inertia of the whole planet suggests a more differentiated internal structure for Mercury. This geophysical quantity improves the constraint on the size of the solid inner core. 


\title{
Planets in a Room: a DIY, low-cost educational kit to teach Planetology
}

\author{
Giacomini L. ${ }^{1,2,3}$, Aloisi F. ${ }^{1}$, De Angelis I. ${ }^{1,4} \&$ Capretti S. ${ }^{5}$ \\ ${ }^{1}$ Speak Science, Rome, Italy. \\ ${ }^{2}$ IAPS-INAF, Rome, Italy. \\ ${ }^{3}$ Europlanet 2020 RI. \\ ${ }^{4}$ Roma Tre University, Dipartimento di Matematica e Fisica, Rome, Italy. \\ ${ }^{5}$ AstronomiAmo, Rome, Italy.
}

Corresponding author email: lorenza.giacomini@inaf.it

Keywords: DIY, educational kit, planetology.

"Planets in a room" is a DIY kit to build a small, lowcost spherical projector that teachers, museum, planetary scientists and other individuals can easily build and use on their own, to show and teach the planets and the stars. The system can also be used under a small dome as a planetairum projector.

The kit was developped by the italian non-profit association Speak Science with the collaboration of INAFIAPS of Rome, the Roma Tre University (Dipartimento di Matematica e Fisica) and the non-profit association AstronomiAmo. The project was funded by the Europlanet Outreach Funding Scheme in 2016 and presented at EPSC 2017 [1] and at EPSC2018 [2].

Starting from September 2018, "Planets in a room" is being distributed to the public thanks to the collaboration of a commercial partner. At the moment, the system is in use in about ten different locations in Europe, including schools, amateur astronomers, Planetarium and other education institutions. The distribution takes place throught the website http://www.planetsinaroom.com/, that is also hosting a community of active users, with the aim of helping the outreach and research community to bring planetary science to a larger audience.

\section{Acknowledgements:}

We acknowledge for this project the vast community of amateur and professionals that is actively working on innovative educational systems for astronomy such as planetarium and virtual reality projects (both hardware and software). Planets in a room is based on the work of this vast community of people and their experiences and results. We also acknowledge for this work Europlanet for funding Speak Science and finally, all scientists, teachers and students who have used (and will use) Planets in a Room.

\section{References:}

[1] Giacomini et al. (2017) - "Planets in a room", EPSC Abstracts Vol. 11, EPSC2017-280, 2017.

[2] Giacomini et al. (2018) - "Planets in a Room: a DIY, low-cost educational kit" EPSC Abstracts Vol. 12, EPSC2018254, 2018. 


\title{
The new Italian Hub of Europlanet Society
}

Giacomini L. ${ }^{1,2}$, De Sanctis M.C. ${ }^{1}$, Ivanovski S.L. ${ }^{3}$, Capria M.T. ${ }^{1}$, Gatti L. ${ }^{4}$, Galluzzi V. ${ }^{1} \&$ Lucchetti A. ${ }^{5}$

\author{
${ }^{1}$ INAF-IAPS. \\ ${ }^{2}$ Europlanet 2020 RI. \\ ${ }^{3}$ INAF- Osservatorio Astronomico di Trieste. \\ ${ }^{4}$ Thales Alenia Space. \\ ${ }^{5}$ INAF- Osservatorio Astronomico di Padova.
}

Corresponding author email: lorenza.giacomini@inaf.it

Keywords: Europlanet Society, planetary science, Italian Hub.

The Europlanet Society is a new membership organisation to promote the advancement of planetary science and related fields, for the benefit of the community.

Built on the heritage of 15 years of Europlanet projects funded by the European Commission, the Europlanet Society was launched in September 2018 at the European Planetary Science Congress (EPSC) in Berlin, and is now open to individual and institutional members.

The Europlanet Society is structured in 10 Regional Hubs that will support the development of planetary science at a national and regional level. The Regional Hubs will organise networking activities and events to support the research community, as well as to build external links with amateur astronomers, industrial partners and policymakers. Outreach is also a core part of Europlanet activities: the Regional Hubs will offer opportunities to engage educators, the media and the wider public with planetary science, sharing best practice on public engagement and education related to planetary science. Their activities are also committed to building a diverse, inclusive planetary science community in Europe and to ensuring that individuals within that community experience equal opportunity.

Italy has a new-born Regional Hub that will be presented at the XV Congresso Nazionale di Scienze Planetarie. In collaboration with SGI, Società Geologica Italiana - Sezione di Geologia Planetaria, the Italian Hub will seize the opportunity of the Congress to launch an online census of the existing groups, infrastructures and initiatives related to Planetary Science in Italy.

Members of the italian community are welcome to subscribe to the Society and contact us to build the next steps of the Europlanet adventure!

To know more about the Italian Europlanet Hub:

http://www.iaps.inaf.it/attivita/italy-europlanet-regional-hub/ 


\title{
Geological mapping of the Kuiper (H06) quadrangle of Mercury
}

\author{
Giacomini L. ${ }^{1}$, Galluzzi V. ${ }^{1}$, Carli C. ${ }^{1}$, Zambon F. ${ }^{1}$, Massironi M. ${ }^{2}$, Ferranti L. ${ }^{3}$ \& Palumbo P. ${ }^{1,4}$ \\ ${ }^{1}$ INAF, Istituto di Astrofisica e Planetologia Spaziali, Rome, Italy; \\ ${ }^{2}$ Dipartimento di Geoscienze, Università degli Studi di Padova, Padua, Italy; \\ ${ }^{3}$ DISTAR, Università degli Studi di Napoli Federico II, Naples, Italy; \\ ${ }^{4}$ Dipartimento di Scienze \& Tecnologie, Università degli Studi di Napoli ‘Parthenope’, Naples, Italy.
}

Corresponding author email: 1orenza.giacomini@inaf.it

Keywords: Mercury, MESSENGER, Kuiper quadrangle.

Kuiper quadrangle (H06) is located at the equatorial zone of Mercury and encompasses the area between longitudes $288^{\circ} \mathrm{E}-360^{\circ} \mathrm{E}$ and latitudes $22.5^{\circ} \mathrm{N}-22.5^{\circ} \mathrm{S}$. In this contribution we present the preliminary results of a more detailed geological map (1:3M scale) of the Kuiper quadrangle based on the higher resolution MESSENGER data.

The main basemap used for mapping is the MDIS (Mercury Dual Imaging System) $166 \mathrm{~m} /$ pixel BDR (map-projected Basemap reduced Data Record) mosaic. Additional datasets were also taken into account, such as DLR stereo-DEM of the region [1], mosaics with high-incidence illumination from the east and west, and MDIS global color mosaic [2]. The preliminary geological map shows that the the western and norther part of quadrangle are characterized by a prevalence of crater materials which were distinguished into three classesbased on their degradation degree [3]. Different plain units were also identified and classified as: (i) intercrater, (ii) intermediate, and (iii) smooth plains. Finally, several structures, mainly represented by thrusts, were mapped all over the quadrangle.

Specific targets of interest, detected during the mapping activity, were studied at higher resolution, integrating geomorphologic and spectral analysis.

The geological map will be merged with the adjoining quadrangles and integrated into the global 1:3M geological map of Mercury [4], which is being prepared in support to BepiColombo mission.

We gratefully acknowledge funding from the Italian Space Agency (ASI) under ASI-INAF agreement 2017-47-H.0. MM, CC, FZ were also supported by European Union's Horizon 2020 research grant agreement No 776276- PLANMAP.

\section{References:}

[1] Preusker et al. (2017) - PSS, 142, 26-37.

[2] Denevi et al. (2018) - Space. Sci. Rev., 214: 2. https://doi.org/10.1007/s11214-017-0440-y.

[3] Galluzzi et al. (2016) - J. Maps, 12, 226-238.

[4] Galluzzi et al. (2018) - Mercury: Current and Future Science of the Innermost Planet. Abstract\#6075. 


\title{
Structural analysis of the north-eastern sector of the Caloris basin
}

\author{
Guzzetta L. ${ }^{1}$, Giacomini L. ${ }^{1}$, Ferranti L. ${ }^{2} \&$ Palumbo P. ${ }^{1,3}$ \\ ${ }^{1}$ INAF - Istituto di Astrofisica e Planetologia spaziali (IAPS), Rome, Italy. \\ ${ }^{2}$ DiSTAR - Dipartimento di Scienze della Terra, dell'Ambiente e delle Risorse, Università "Federico II”, Naples, Italy. \\ ${ }^{3}$ Dipartimento di Scienze \& Tecnologie, Università degli Studi di Napoli "Parthenope", Naples, Italy.
}

Corresponding author email: laura.guzzetta@inaf.it

Keywords: Mercury, MESSENGER, Caloris basin, Structural analysis.

The tectonic landforms located in the north-eastern sector of the Caloris basin, the largest impact crater on Mercury, have been analysed by using the WAC and NAC MDIS (Mercury Dual Imaging System) images acquired by the NASA MESSENGER mission. The majority of these features are located in the H03 quadrangle of Mercury, whose 1:3M-scale geological map was recently published [1] and in which they were generally mapped as thrusts or wrinkle ridges. Here, we have distinguished the structures according to the scarp geometry as linear or arcuate. Azimuthal analyses have been performed on both class of structures and show two different preferential orientations that led us to suppose more than a single deformation stage. The "buffered crater-counting" technique [2] has been performed to estimate the absolute age of the linear and arcuate structures using the structure-superimposed impact craters density. The resulting CSFD (cumulative size-frequency distribution) diagrams show ages of $3.7 \mathrm{Gyr}$ by applying the production function of Le Feuvre and Wieczorek [3], and 3.8 Gyr by using Neukum's production function [4], for both sets of structures. Further, the main terrain units affected by the linear and arcuate structures have been dated by using the crater counting method. In order to understand the possible interaction between these structures and the Caloris formation, the analysis will be extended to whole area around the basin, with particular focus for the structures occurring at the south-western sector over the edge of Caloris. Understanding the local deformation pattern and timing of faulting can contribute to improve the knowledge of the past stress state of the planet.

We gratefully acknowledge funding from the Italian Space Agency (ASI) under ASI-INAF agreement 2017-47-H.0.

References:

[1] Guzzetta et al. (2017) - J Maps.

[2] Tanaka (1982) - Planet. Geol. and Geoph. Progr.

[3] Le Feuvre \& Wieczorek (2011) - Icarus.

[4] Neukum et al. (2001) - Planet. Space Sci. 


\title{
Modelled magnetic reconnection at the Mercury's magnetopause in support of the scientific objectives of SERENA
}

\author{
Ivanovski S.L. ${ }^{1}$, Milillo A. ${ }^{2}$, Kartalev M. ${ }^{3}$, Massetti S. ${ }^{1}$, Orsini S. ${ }^{2}$, Sordini R. ${ }^{2}$, Mura A. ${ }^{2}$, Mangano V. ${ }^{2}$, \\ De Angelis E. ${ }^{2}$, Rispoli R. ${ }^{2}$, Lazzarotto F. ${ }^{2}$, Aronica A. ${ }^{2} \&$ Kazakov A. ${ }^{2}$ \\ ${ }^{1}$ INAF-Osservatorio Astronomico di Trieste, via Giambattista Tiepolo, 11, 34143 Trieste, Italy. \\ ${ }^{2}$ INAF-IAPS, via Fosso del Cavaliere 100, 00133 Rome, Italy. \\ ${ }^{3}$ Geospace Consult Ltd, Institute of Mechanics, Bulgarian Academy of Sciences, Acad. G. Bontchev St., bl. 4, \\ 1113 Sofia, Bulgaria.
}

\section{Corresponding author email: stavro.ivanovski@,inaf.it}

Keywords: Mercury, BepiColombo, Magnetopause.

The ESA/JAXA BepiColombo mission was launched successfully in October 2018 and among its scientific objectives is the investigation of the physical conditions in which magnetic reconnection occurs. KelvinHelmholtz (KH) waves and magnetic reconnection are believed to be the key drivers of plasma transport and planetary magnetospheres are excellent laboratories to investigate them. In the preparation of the scientific phase of BepiColombo, as a first step, we use two MESSANGER datasets for investigating the development of MHD instabilities as a possible mechanism causing magnetic reconnection phenomena at Mercury's magnetopause. Based on a flexible numerical incompressible magnetohydrodynamic (MHD) approach utilized for studying coupled Kelvin-Helmholtz (KH) and tearing mode (TM) instabilities [1], we investigate the applicability of such mixing-boundary-layer MHD approach to the Mercury's magnetopause and by means of numerical simulations we explore the model parameter space using the MESSENGER data [2]. In the case of high-shear magnetopause reconnection, for example on 24 November 2011, we obtained the typical vortex features that exhibit magnetic reconnection in the developed $\mathrm{KH}$ and TM instabilities. In the case of low-shear magnetopause reconnection, for example on 21 November 2011, we obtained no manifestation of magnetic reconnection. We discuss the scientific applicability of this approach to support the scientific objectives of SERENA experiment [3] onboard BepiColombo, providing information on the coupling processes that occur in the Mercury complex system surface-exosphere-magnetosphere.

\section{Acknowledgements:}

This work is supported by the SERENA ASI-INAF agreement.

\section{References:}

[1] Ivanovski et al. (2011) - JTAM, vol. 41, No. 3, pp. 31-42.

[2] DiBraccio et al. (2013) - JGR, 118, 997-1008

[3] Milillo et al. (2005) - SSR. 


\title{
Global properties of hollows on the surface of Mercury
}

\author{
Lucchetti A. ${ }^{1}$, Pajola M. ${ }^{1}$, Semenzato A. ${ }^{2}$, Galluzzi V. ${ }^{3}$, Munaretto G..${ }^{1,4}$, Cremonese G. ${ }^{1}$, \\ Massironi M. ${ }^{1,2} \&$ Palumbo P. ${ }^{5}$ \\ ${ }^{1}$ INAF-Astronomical Observatory of Padova, Italy. \\ ${ }^{2}$ Geosciences Department, University of Padova, Italy. \\ ${ }^{3}$ INAF-IAPS Roma, Istituto di Astrofisica e Planetologia Spaziali di Roma, Italy. \\ ${ }^{4}$ Physics and Astronomy Department, University of Padova, Italy. \\ ${ }^{5}$ Dipartimento di Scienze e Tecnologie, Università di Napoli "Parthenope".
}

Corresponding author email: alice.lucchetti@inaf.it

Keywords: Mercury, MESSENGER, Hollows.

"Hollows were revealed for the first time on the surface of Mercury as shallow irregular and rimless flatfloored depressions with bright interiors and halos, often found on crater walls, rims, floors and central peak $[1,2,3]$.

Hollows features are located everywhere on the surface of the planet [3] and since they are fresh in appearance, they may be actively forming today via a mechanism that could involve depletion of subsurface volatiles $[1,4]$ whose nature is not known.

In this work, combining different data acquired by the MESSENGER mission, we investigate the global properties of hollows located in different geochemical terrains of the planet. We analyse the distribution of hollows with respect to elevation data, gravity data, craters distribution and crustal thickness mainly focusing our work on the high Mg-region of Mercury. In addition, to describe in greater detail the nature of hollows and their origin, we locally investigate the correlation between hollows and other geological evidences, such as pyroclastic deposits. We performed it through both a geomorphological and a spectral analysis identifying the behaviour of these particular structures when occurring with pyroclastic deposits. Finally, we compare these results with the behaviour of isolated hollows [5].

\section{Acknowledgements:}

We gratefully acknowledge funding from the Italian Space Agency (ASI) under ASI-INAF agreement 2017- 47-H.0.

\section{References:}

[1] Blewett et al. (2011) - Science, 333, 1856-1859.

[2] Blewett et al. (2013) - JGR Planets, 118, 1013-1032.

[3] Thomas et al. (2014) - Icarus, 229, 221-235.

[4] Vaughan et al. (2012) - LPSC, 43, abstract 1187.

[5] Lucchetti et al. (2018) - JGR. 


\title{
Surface geology of Arsinoes Chaos: morphological and spectral affinities across Chaotic terrains on Mars
}

\author{
Luzzi E. ${ }^{1}$, Rossi A.P. ${ }^{1}$, Carli C. ${ }^{2} \&$ Altieri F. ${ }^{2}$ \\ ${ }^{1}$ Jacobs University Bremen. \\ ${ }^{2}$ INAF-IAPS Istituto di Astrofisica e Planetologia Spaziali.
}

Corresponding author email: e.luzzi@jacobs-university.de

Keywords: Arsinoes Chaos, Aram and Aureum Chaos, Mars, CTX and HiRISE experiments

We are mapping surface units on Arsinoes Chaos (Mars) based on morphological observations from CTX and HiRISE experiments, as well as through spectral analyses of CRISM data. Strong analogies in the depositional, erosional and collapse history suggest that the processes acting in Arsinoes Chaos may be the same responsible for the formation of other Chaotic terrains such as Aram Chaos [1] and Aureum Chaos [2]. Aram and Aureum Chaos are characterized by a basaltic bedrock disrupted into kilometric polygonal blocks and knobs, locally carved by outflow channels and stratigraphically underlying sedimentary units rich in sulfates. Several interprations were proposed in literature for the origin of such features, as summarized by [3]; the common point between all the scenarios is the occurrence of a catastrophic collapse and consequent outflow. In Arsinoes Chaos, in addition to the typical bedrock disruption in polygonal blocks and knobs, also the layered sedimentary fill displays morphological affinities with those occurring in the other Chaotic terrains. If the spectral analyses will confirm the mineralogical analogy between Arsinoes, Aram and Aureum Chaos, we expect to observe the spectral signature of hydrated minerals (such as sulfates), plus possibly hematite. The depositional environment inferred for the sedimentary units in Aram Chaos [e.g. 1] is water-rich with possible evidences of hydrothermal or diagenetic processes. We are investigating the possible regional correlations between Chaotic terrains, as well as the possible variability of tectonic and depositional processes within and across them.

References:

[1] Glotch \& Christensen (2005) - JGR-Planets, 110(E9).

[2] Sowe et al. (2012) - Icarus, 218(1), 406-419.

[3] Roda et al. (2014) - Icarus, 236, 104-121. 


\section{Moon Farside Radiotelescope and its Protection}

Maccone C.

IASF-INAF Milano.

Corresponding author email: clmaccon@libero.it

Keywords: Moon farside, Radiotelescope, Radioastronomy.

The Moon Farside is the only place in space, and not too far from the Earth, where radio transmissions and noises produced by Humanity on Earth may not reach since the spherical body of the Moon blocks them, acting like a shield. On June 10, 2010, this author was the first scientist to present the case for the Moon Farside Protection at the United Nations Office of Outer Space Affairs in Vienna during a meeting of UN-COPUOS, the United Nations Committee on the Peaceful Uses of Outer Space. Unfortunately, the undeclared but quite real "current, new race to the Moon" complicates matters terribly. All the space-faring nations now keep their eyes on the Moon, and only the United Nations might have a sufficient authority to Protect the Farside and keep safe its unique "radio-noise free" environment. But time is money, and the "Moon Settlers" may well reach the Moon before the United Nations come to agree about any official decision concerning the Farside Protection. Quite an URGENT ISSUE. In this paper, we firstly define the PAC ( $1 / 4$ Protected Antipode Circle), i.e. a circular piece of land on the Farside having its center at the Antipode of the Earth and tangent to the 300 and -300 parallels. This turns out measuring about $1820 \mathrm{~km}$ in diameter on the surface of the Moon Farside. Then we propose that the new "Moon Village", supported by the vision of the ESA Director General, Jan Woerner, be located OUTSIDE the PAC (obviously not to interfere with the detection of radiation coming from space) and also SOUTH OF THE PAC, to be "close" to the South Pole as much as needed in order to benefit of frozen water there. It thus appears that the best venue for the "Moon Village" would be on or around the 1800 meridian and possibly quite close to the South Pole. 


\title{
Clay formation in Margaritifer Chaos, Mars
}

\author{
Marinangeli L. ${ }^{1}$, Tangari A.C. ${ }^{1}$, Pompilio L. ${ }^{1}$, Scarciglia F. ${ }^{2}$ \& Piluso E. ${ }^{2}$ \\ ${ }^{1}$ Laboratorio di Telerilevamento e Planetologia, DiSPUTer, Universita' G. d'Annunzio, via dei Vestini 31, 66013 Chieti. \\ ${ }^{2}$ Dipartimento di Biologia, Ecologia e Scienze della Terra (DiBEST), Università della Calabria, , 87036 Arcavacata di \\ Rende, Cosenza.
}

Corresponding author email: 1ucia.marinangeli@unich.it

Keywords: Clay formation, Mars, smectite group.

Clay formation processes on Mars is thought to have been occurred early in its geological history, however reactivation of slope features (RSL) likely due to brine melting during the summer season has been extensively observed on recent data (Muskin, 2010; McEwen, 2014). This may also indicate that chemical alteration of bedrock still occurs at places. While we found the typical spectral adsorption bands of Al-rich clays, such as illite and montmorillonite belonging to the smectite group in Valles Marineris by using the CRISM hyperspectral data, a different clays assemblage was observed in Margaritifer Chaos, a complex fluvial system nearby Valles Marineris. The spectral signature of allophane (poorly crystalline clay), vermiculite, chlorite and other phyllosilicates belonging to the $\mathrm{Fe} / \mathrm{Mg}$ smectite group, such as saponite and nontronite characterise the Margaritifer study area. This may suggest either a different alteration origin or an overlapping of different depositional processes. Polygons features are often associated to the clay signatures, suggesting the occurrences of desiccation processes.

On the contrary, large surrounding areas are dominated by basaltic composition with no evidence of diagnostic bands of clays or other phillosylicates, indicating the scale-dependency of these sedimentary events. 


\title{
Exospheric Na distributions along the Mercury orbit with the THEMIS telescope
}

\author{
Milillo A., Mangano V., Massetti S., Mura A., De Angelis E., Ivanovski S.L., Orsini S., Rispoli R., \\ Lazzarotto F., Aronica A. \& Kazakov A.
}

INAF, Istituto di Astrofisica e Planetologia Spaziali, Roma.

Corresponding author email: anna.milillo@inaf.it

Keywords: Mercury orbit, Na distribution, THEMIS telescope.

The Mercury's Na exosphere observations obtained by ground based observations and by space observations seem to lead to different scenarios of the interaction between the environment and the planet. In fact, the ground-based observations show often a double peak at mid-high latitudes with time scale variations less than one hour not related to any surface feature nor to season, while the MESSENGER/MASCS observations have a strong seasonal dependence and do not evidence a short time-scale variability. For this reason, it has to be investigated whether the Sun's activity is the major driver of the Na exosphere configuration at Mercury, or the variations are only due to the surface temperatures considered in long time scales or on position along the orbit. In this paper, in order to better investigate this open issue, we have studied the local time and latitudinal distributions of the exospheric Na column density as a function of the True Anomaly Angle (TAA) of Mercury by means of the extended dataset of images, collected from 2009 to 2013, by the THEMIS solar telescope. In particular, THEMIS images, in agreement with the MESSENGER data, registered a strong dawnward emission predominance with respect to duskward and subsolar region between $90^{\circ}$ and $150^{\circ}$ TAA. Also, an unexpected relation between Northward or Southward peak emission and both TAA and local time is evidenced by our analysis, requiring further investigations. 


\title{
Power spectral analysis of Jupiter's polar clouds by JIRAM data
}

\author{
Moriconi M.L. ${ }^{1}$, Adriani A. ${ }^{2}$, Grassi D. ${ }^{2}$, Mura A. ${ }^{2}$, Altieri F. ${ }^{2} \&$ the JIRAM team \\ ${ }^{1}$ ISAC-CNR, Via Fosso del Cavaliere 100, 00133 Roma. \\ 2 IAPS-INAF, Via Fosso del Cavaliere 100, 00133 Roma.
}

Corresponding author email: m.moriconi@isac.cnr.it

Keywords: Jupiter, Juno mission, Jupiter polar clouds, JIRAM.

We analyze the pattern of Jupiter's polar clouds, as acquired by JIRAM (Jupiter InfraRed Auroral Mapper) during some of Juno orbits, in order to investigate the characteristics of the brightness power spectra in relationship with Jupiter's dynamics and turbulence. This study is based on the images acquired by the JIRAM imager in M band (IMG-M hereafter), centered at $4.78 \mu \mathrm{m}$ with a $480 \mathrm{~nm}$ bandwidth, and has been carried out on both poles. This observation wavelength probes thermal emission from $\sim 5$ bar level, like reported by Harrington et al. (1996); the overlying clouds attenuate the emission and we concentrate on the power spectrum of the related opacities to characterize the cloud pattern statistics and its changes with the increasing orbits. We investigate also length scales for possible energy deposition. However, while South Pole full coverage could be obtained during many of the orbits, the same were not possible about the North Pole due to the spacecraft attitude not always favorable to JIRAM observations. We extracted brightness signal scans by the image mosaics as circular paths. The circular paths have been shaped on latitude circles, and then translated to cover regions showing a homogeneous dynamical appearance, typically outside and inside the circumpolar cyclones ring. We computed power spectra from these spatial data records using Fast Fourier Transform analysis. Power spectra so computed resemble theoretical spectra of two-dimensional fluid turbulence with the first power law slope ranging in the -0.9/-1.45 interval (low wavenumbers) and the second slope in the -3.0/-3.9 (high wavenumbers). If a baroclinic instability can be assumed in the polar cap area, as the change in the slope of the power spectra seems indicate, an energy input at planetary wavenumber $\sim 100$ can be hypothesized for the outer region of the circumpolar cyclones. For the inner region, on the contrary a so clear scale input (planetary wavenumber variable in the 20-50 range) is not present. 


\title{
Jupiter's Magnetosphere from Juno
}

\author{
Mura A. ${ }^{1}$, Adriani A. ${ }^{1} \&$ Bolton S. ${ }^{2}$ \\ ${ }^{1}$ INAF-IAPS, Italy. \\ ${ }^{2}$ SwRI, USA.
}

Corresponding author email: alessandro.mura@gmail.com

Keywords: Jupiter, Juno mission, Magnetosphere.

NASA's Juno mission has been observing the Jovian magnetosphere since 2016 from a polar, highly elliptical orbit. From this very favourable position above the poles, Juno obtained unprecedented in-situ measurements of Jupiter magnetosphere, and unique, remote-sensing views of the auroral regions. Hence, Juno has put a milestone in the understanding of the planet magnetosphere studies. Here we presents the main results from the magnetospheric/auroral experiments on board Juno: the Jovian InfraRed Auroral Mapper, (JIRAM), the Ultra Violet camera (UVS), and the plasma sensors (JADE, JEDI).

In the main oval, the field aligned electric currents have very low densities, considerably weaker than what was previously assumed to explain the intense auroral emissions. It is now believed that stochasticlike broadband acceleration is the dominant process, with respect to steady magnetic field-aligned electric potentials as at the Earth. Juno observed the high energy ( $\left.\_100 \mathrm{keV}\right)$ auroral electron, but also a lower energy component $(\sim 1 \mathrm{keV})$, which plays a substantial role. Inside the main oval, persistent upward broadband highenergy $(\sim 1 \mathrm{MeV})$ electron are observed. Also, intense auroral emissions are observed in regions where little or no precipitating electron flux is detected, possibly indicating excitation from strong ionospheric electric currents.

Juno also revealed a puzzling structure in the electromagnetic interactions between Jupiter and its moons, whose signature is the presence of auroral footprints. These are a peculiar series of emission features extending downstream of the leading one, and look like a repeating pattern of swirling vortices. These multiple features have a very small scale $(\sim 100 \mathrm{~km})$, which is not compatible with the simple paradigm of multiple Alfven wave reflections. Observations of Io's footprint well downstream of the leading feature reveal a pair of closely spaced parallel arcs where previously only one was resolved.

Both of Ganymede's footprints (main and secondary) appear as a pair of emission features that provide a remote measure of Ganymede's magnetosphere. 


\title{
Mars dust characterization by means of TGO/NOMAD data analysis
}

Oliva F. ${ }^{1}$, Bellucci G. ${ }^{1}$, D’ Aversa E. ${ }^{1}$, Altieri G. ${ }^{1}$, Carrozzo F.G. ${ }^{1}$, Daerden F. ${ }^{2}$, Depiesse C. ${ }^{2}$, Lopez-Moreno J.-J. ${ }^{3}$, Mason J., Patel M.R., Ristic B. ${ }^{2}$, Thomas I.R. ${ }^{2}$, Vandaele A.C. ${ }^{2}$ \& Willame Y. ${ }^{2}$

\author{
${ }^{1}$ INAF-IAPS, Rome, Italy. \\ ${ }^{2}$ IASB-BIRA, Bruxelles, Belgium. \\ ${ }^{3}$ IAA-CSIC, Granada, Spain. \\ ${ }^{4}$ The Open University, Milton Keynes, United Kingdom.
}

Corresponding author email: fabrizio.oliva@inaf.it

Keywords: Mars, Exomars, Dust, NOMAD.

The Nadir and Occultation for MArs Discovery spectrometer (NOMAD, [1]), onboard the Trace Gas Orbiter (TGO) spacecraft, is currently observing Mars atmosphere. The instrument is capable to observe with different pointing geometries taking advantage of three channels: UVIS, operating in the ultraviolet/visible range $0.2-0.65 \mu \mathrm{m}$, working both in nadir and solar occultation geometries; LNO, covering the infrared range $2.3-3.8 \mu \mathrm{m}$, working in nadir, limb and solar occultation geometries; SO, working in the range $2.3-4.3 \mu \mathrm{m}$ and performing dedicated solar occultation measurements. Although NOMAD has been mainly conceived to study the trace gases in the atmosphere of the red planet, it can also provide valuable information regarding the properties of Martian dust. In this work we will show ongoing studies on the datasets of all NOMAD channels, focused on the characterization of the geometrical and microphysical properties of dust, highlighting the most critical issues in the framework of such analyses.

\section{Acknowledgements:}

The NOMAD experiment is led by the Royal Belgian Institute for Space Aeronomy (IASB-BIRA), assisted by Co-PI teams from Spain (IAA-CSIC), Italy (INAF-IAPS), and the United Kingdom (Open University). This project acknowledges funding by the Belgian Science Policy Office (BELSPO), with the financial and contractual coordination by the ESA Prodex Office (PEA 4000103401, 4000121493), by Spanish MICINN through its Plan Nacional and by European funds under grant ESP2015-65064-C2-1-P (MINECO/FEDER), as well as by UK Space Agency through grants ST/R005761/1, ST/P001262/1, ST/R001405/1 and ST/R001405/1 and Italian Space Agency through grant 2018-2-HH.0.

\section{References:}

[1] Neefs et al. (2015) - Applied Optics Vol. 54, No. 28, 8494-8520. 


\title{
Radar evidence of subglacial liquid water on Mars
}

\author{
Orosei R. ${ }^{1}$, Lauro S. E. ${ }^{2}$, Pettinelli E. ${ }^{2}$, Cicchetti A. ${ }^{3}$, Coradini M. ${ }^{4}$, Cosciotti B. ${ }^{2}$, Di Paolo F. ${ }^{1}$, Flamini E. ${ }^{4}$, \\ Mattei E. ${ }^{2}$, Pajola M. ${ }^{5}$, Soldovieri F. ${ }^{6} \&$ Cartacci M. ${ }^{3}$ \\ ${ }^{1}$ Istituto Nazionale di Astrofisica, Istituto di Radioastronomia. \\ ${ }^{2}$ Università degli Studi Roma Tre, Dipartimento di Matematica e Fisica. \\ ${ }^{3}$ Istituto Nazionale di Astrofisica, Istituto di Astrofisica e Planetologia Spaziali. \\ ${ }^{4}$ Agenzia Spaziale Italiana. \\ ${ }^{5}$ Istituto Nazionale di Astrofisica, Osservatorio Astronomico di Padova. \\ ${ }^{6}$ Consiglio Nazionale delle Ricerche, Istituto per il Rilevamento Elettromagnetico dell'Ambiente.
}

Corresponding author email: roberto.orosei@inaf.it

Keywords: Mars, Polar caps, Mars Express, MARSIS, Radar sounding.

The presence of liquid water at the base of the Martian polar caps has long been suspected but not observed. We surveyed the Planum Australe region using the Mars Advanced Radar for Subsurface and Ionosphere Sounding, a low-frequency radar on the Mars Express spacecraft. Radar profiles collected between May 2012 and December 2015, contain evidence of liquid water trapped below the ice of the South Polar Layered Deposits. Anomalously bright subsurface reflections were found within a well-defined, $20 \mathrm{~km}$ wide zone centered at $193^{\circ} \mathrm{E}, 81^{\circ} \mathrm{S}$, surrounded by much less reflective areas. Quantitative analysis of the radar signals shows that this bright feature has high dielectric permittivity _ 15, matching water-bearing materials. We interpret this feature as a stable body of liquid water on Mars. 


\title{
ExoMars 2020 Oxia Planum landing area characterization and performance assessment for Surface Platform landing, Rover egress and traversability
}

\author{
Pacifici A. ${ }^{1}$, Ori G.G. ${ }^{1}$, Aboudan A. ${ }^{1}$, Murana A. ${ }^{1}$, Di Pietro I. ${ }^{1}$ \& Lorenzoni L. ${ }^{2}$ \\ ${ }^{1}$ IRSPS, Università d'Annunzio, Viale Pindaro 42, 65127, Pescara, Italy. \\ ${ }^{2}$ European Space Agency, ESTEC, Noordwijk, Netherlands.
}

Corresponding author email: pacifici@,irsps.unich.it

Keywords: Mars, Exomars, Dust, Oxia Planum.

Oxia Planum landing site has been recently selected for the ExoMars 2020 mission on Mars of the European Space Agency and Roscosmos, with Thales Alenia Space Italia (TAS-I) and NPO Lavochkin as prime contractor. In the framework of the Environmental Terrain Support Analysis, we investigated the Oxia Planum area in order to evaluate the landing and the egress feasibility for the descent module (DM) and the trafficability performance of the ExoMars 2020 Rover.

At the base of this analysis there is a Surface Characterization Map of the investigated area that have been realized from the DM landing and Rover mobility perspective. The database utilized for the mapping consist of MOLA DEM, THEMIS IR Day and Night mosaics, HRSC images and DEM (and derived slope maps), CTX images, HiRISE images and DEMs (and derived slope maps). All this data has been ingested, co-registered and analyzed through a GIS software. Mapping of different surface typologies have been performed with the aim of to map the distribution of areas with similar surface characteristics. Afterwards, using a novel approach for landing site analysis, the Surface Characterization Map has been used in order to infer the compliance of each unit w.r.t. meter and sub-meter landing site engineering constraints (e.g. meter-scale slopes and rock abundance) overall the entire landing characterization area. Meter and sub-meter information, in fact, are available only on selected areas (depending on HiRISE images location).

Our results indicate a full compliance of the landing region terrain for latitude, elevation, albedo and thermal inertia constraints. Slope analysis at kilometers, hectometers and meters scale show areas of non compliance generally below $5 \%$ of the characterization area. Rocks and boulders abundance analysis appears preliminarly compliant and more detailed analysis are on-going. We thank F. Calantropio and A. Merlo of Thales Alenia Space Italy for their fundamental contribution to this work. 


\section{Mars Human Exploration: the Vernal crater landing site}

Pajola M. ${ }^{1}$, Pozzobon R. ${ }^{1,2}$, Simioni E. ${ }^{1}$, Teodoro L. ${ }^{3}$, Wilson J. ${ }^{4}$, Kling A. ${ }^{5}$, Salese F. ${ }^{6,7}$, Lucchetti A. ${ }^{1}$, Munaretto G. ${ }^{1,8}$, Massironi M. ${ }^{1,2}$, Cremonese G. ${ }^{1}$, Sauro F. ${ }^{9}$, Silvestro S. ${ }^{10,11}$, Marinangeli L. ${ }^{12}$, Pompilio L. ${ }^{12}$, Tangari A.C. ${ }^{12}$, Noe Dobrea E. ${ }^{5} \&$ De Toffoli B. ${ }^{2}$

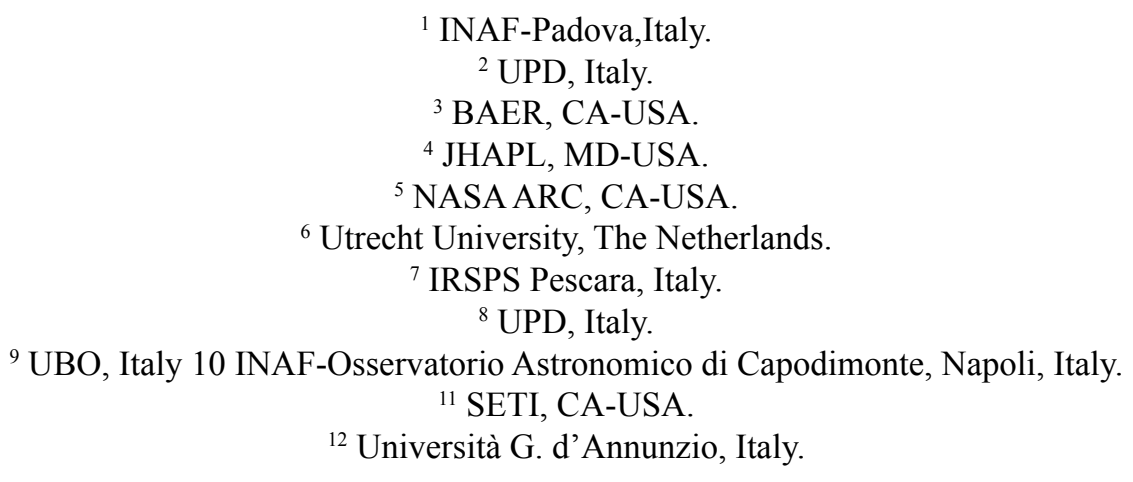

Corresponding author email: maurizio.pajola@inaf.it

Keywords: Mars, Human exploration, Landing site.

The identification of a Mars lander or rover landing site is a multi-step process that involves both the fulfilment of several safety engineering constraints (latitude, elevation, surface slopes, rock abundances, thermal inertia, albedo and radar reflectivity) as well as scientific requirements. Based on the search for past conditions suitable for life, identification of Mars rover landing spots have prioritized regions with subaqueous or hydrothermal sediments coupled with morphological criteria for past standing bodies of water, i.e. seas, rivers, glaciers. The above mentioned criteria still hold for Human exploration, but, in addition to that, there is the added pivotal requirement of in situ identification of exploitable resources, such as reachable subsurface water ice reservoirs, aiming to sustain a settlement of human beings. In this work we are going to show the analysis we performed on a specific area of Mars located North of Meridiani Planum and enclosing the Vernal crater $\left(6^{\circ} \mathrm{N}, 355.5^{\circ} \mathrm{W}\right)$. After deeply evaluating the fulfilment of the engineering requirements, we present the scientific rationale characterising this proposed future Human landing site.

Acknowledgments:

This research is supported by the Italian Space Agency (ASI) within the ASI-INAF agreement no. 2017-03-17. 


\title{
Planetary geological mapping: integrating Earth-based protocols with the support of the Italian Geological Survey
}

\author{
Pantaloni M. ${ }^{1}$, Graciotti R. ${ }^{1}$, Roma M..${ }^{1}$, Carta R. ${ }^{1}$, D’Orefice M. ${ }^{1}$, Campo V. ${ }^{1}$, Pondrelli M. ${ }^{2}$, Marinangeli L. ${ }^{2}$, \\ Massironi M. ${ }^{3}$, Pozzobon R. ${ }^{3}$, Ferrari S. ${ }^{3}$, Carli C.. , Galluzzi V. ${ }^{4}$, Ferranti L. ${ }^{5}$, Mancinelli P. ${ }^{6}$, Pauselli C. ${ }^{6}$, \\ Melis M.T. ${ }^{7}$, Rossi A.P. ${ }^{8}$ \& Zinzi A. ${ }^{9}$ \\ ${ }^{1}$ Geological Survey of Italy - ISPRA, Rome. \\ ${ }^{2}$ Università d'Annunzio, Chieti-Pescara. \\ ${ }^{3}$ Department of Geosciences, Università di Padova. \\ ${ }^{4}$ Istituto di Astrofisica e Planetologia Spaziali -INAF, Rome. \\ ${ }^{5}$ Dipartimento di Scienze della Terra, dell'Ambiente e delle Risorse, Università di Napoli "Federico II". \\ ${ }^{6}$ Dipartimento di Fisica e Geologia, Università di Perugia. \\ ${ }^{7}$ Department of Chemical and Geological Sciences, Università di Cagliari. \\ ${ }^{8}$ Jacobs University, Bremen. \\ ${ }^{9}$ Space Science Data Center - Italian Space Agency, Rome.
}

Corresponding author email: marco.pantaloni@isprambiente.it

Keywords: Geological-geomorphological mapping, Planetary datasets, MATISSE.

Although based on the same basic principles as on Earth, planetary geological-geomorphological mapping has some peculiarities which needs to be addressed in order to standardize the technical and scientific approach.

Planetary mapping is data-dependent (i.e., images resolution, availability of spectral data, etc.), which implies differences between planets but also between different parts of the same planet. Furthermore, groundtruth is generally unavailable (with the notable exception of the Moon and the rover missions mostly on Mars) and, sometimes, new data acquisition can dramatically change the geological analyses in planetary bodies as well as differences in mapping products. These problems, combined to the fact that planetary geology is a new discipline compared to terrestrial geology, contribute to create confusion both in the map realization and representation. The need to address these issues and to update the current protocols of the US Geological Survey, has been also highlighted by a recent H2020 project, PlanMap, led by M. Massironi (https://planmap. $\underline{\mathrm{eu}}$ ). Furthermore, a comprehensive data archive and visualization tool to retrieve different planetary datasets is developed by the ASI Space Science Data Center (MATISSE project https://tools.ssdc.asi.it/matisse.jsp). The involvement of cartographic offices in this activity is fundamental to identify mapping guidelines that might become standards. The Geological Survey of Italy is among the oldest cartographic service in Europe and has the required knowledge to help in correctly map the geological and geomorphological units and related symbols.

We create a multidisciplinary working group aimed to test the existing mapping protocols for planetary cartography (i.e., legend definition and symbols from USGS and PlanMap) with terrestrial-based cartographic standards. We present preliminary results of a martian study case and evaluate potential implications in the revision of the mapped geological units. 


\title{
Toward a simulation of ice-covered oceans
}

\author{
Provenzale A. ${ }^{1} \&$ Lenzi S. ${ }^{1}$ \\ ${ }^{1}$ Institute of Geoscienzes and Earth Resources, CNR, Pisa.
}

Corresponding author email: antonello.provenzale@enr.it

Keywords: Ganymede, JUICE mission, Ocean, Icy crust.

The observations of Ganymede support the presence of a stratified structure for the largest satellite of Jupiter, with the presence of an ocean of liquid (salty) water below a surface ice crust. Radioactive decay from the lower rocky mantle, and, to a lesser extent, tidal friction are considered the main drivers of the vertical heat flux which can generate convection processes both within the ice and the ocean, albeit with very different time scales. For this reason, the vertical heat transport across the ocean, from the bottom high-pressure ice to the surface crust, is one of the interesting variables because of its effects on the global energy balance and on the depth of the surface ice crust. Here we provide a preliminary description of the set-up for the simulation of such processes, based on a convective fluid dynamical model in a locally Cartesian approximation of a portion of the ice-covered ocean. To this aim, we pay special attention to the heat exchange at the upper ocean-ice interface. Since new measurements will be available from the JUICE space mission, future observational data will allow to test and tune the numerical models. 


\title{
The intriguing case of Ladon Vallis, Mars
}

\author{
Pompilio L. ${ }^{1} \&$ Marinangeli L. ${ }^{1}$ \\ ${ }^{1}$ DISPuTer, Università G. d'Annunzio, Via dei Vestini, 31, Chieti, Italy.
}

Corresponding author email: loredana.pompilio@unich.it

Keywords: Mars, Surface composition, MRO mission, HiRISE, Ladon Vallis.

To have an understanding of the geological evolution of Mars, we need to make feasible inferences on the composition of the exposed terrains, at least.

The geological surfaces observed remotely by orbiters are likely composed of mixtures of minerals, and rocks, as well. Therefore, the spectral response in each pixel can reasonably be considered as the linear combination of the spectra of all the component endmembers. Multiple endmember spectral mixture analysis (MESMA), which is an evolution of the most known Spectral Mixture Analysis (SMA), allows the number and types of endmembers to vary on a pixel base, while the minimum RMSE of the fitting model is accomplished [1].

Ladon Vallis is certainly an interesting target to study and a geologically intriguing place of Mars. CTX and HiRise data show features resembling both huge mud flows coming out of a complex channel system and few pulses of lava flows coming out of fissural eruptions in a graben system. Also some light toned deposits outcrop in places. Are they salts resulting from evaporation? Or mineral assemblages due to hydrothermal processes?

To unravel this mystery, the spectral information and MESMA analysis could help, in principle. Therefore, we tested a number of combinations using the spectra available within known libraries, and tried to provide evidence of minerals and rocks distribution at the surface. We used CRISM data available in the area.

\section{References:}

[1] Gou (2015) - Geoscience frontiers. 


\title{
The CaSSIS data management and visualization on ASI-SSDC
}

\author{
Re C. ${ }^{1}$, Zinzi A. ${ }^{2}$, Cremonese G. ${ }^{1}$, Simioni E. ${ }^{1}$, Mudric T. ${ }^{1} \&$ Capria M.T. ${ }^{3}$ \\ ${ }^{1}$ OAPD-INAF, Padova, Italy. \\ ${ }^{2}$ Space Science Data Center - ASI, Roma, Italy. \\ ${ }^{3}$ IAPS-INAF, Roma, Italy.
}

Corresponding author email: cristina.re@inaf.it

Keywords: Mars, Exomars TGO, CaSSIS.

The Colour and Stereo Surface Imaging System (CaSSIS) [1] on the ExoMars Trace Gas Orbiter (TGO) is a multi-spectral stereo camera based on the push-frame acquisition. CaSSIS is the European stereo acquisition system with the highest resolution operating around Mars. The experience of the Italian team of SIMBIOSYS [2] in stereo cameras, for what concerns the 3D reconstruction of planetary surfaces, has contributed to establish its leadership for the Digital Terrain Model (DTM) reconstruction [3] and 3D data archiving [4] within the CaSSIS international team. The availability of high resolution DTMs, in combination with colour images, introduces the possibility of an efficient data-fusion oriented to more accurate geological maps and other specific geo-morphological investigations. The creation of a pipeline to mosaic the stereo images allowing the production of the three-dimensional data by means of a photogrammetric process could be a key point for the introduction of CaSSIS data inside MATISSE [5]. Thanks to this tool, developed by the Space Science Data Center (SSDC-ASI) and now facing a major upgrading phase, it will be possible to: a) select CaSSIS images with geographical/geometrical metadata queries to a GIS-designed database; b) generate high-order products with data fusion capabilities (e.g., mosaics, ratios); c) visualize 3D data projections using high-resolution DTMs. The synergy here proposed between two very advanced items could be of fundamental importance in enhancing the scientific return, with obvious benefits for all the interested community.

\section{References:}

[1] Thomas et al. (2017) - Space Science Reviews.

[2] Flamini et al. (2010) Planetary and Space Sciences.

[3] Simioni et al. (2017) ISPRS, XLII-3/W1.

[4] Cremonese et al. (2018) - EPSC2018-1236.

[3] Zinzi et al. (2016) - Astronomy \& Computing. 


\title{
Mercury surface temperature distribution
}

\author{
Rognini E. ${ }^{1}$, Capria M.T. ${ }^{1} \&$ Zinzi A. ${ }^{2}$ \\ ${ }^{1}$ INAF-IAPS Istituto di Astrofisica e Planetologia Spaziali, Via del Fosso del Cavaliere 100, 00133 Roma. \\ ${ }^{2}$ ASI Science Data Center (ASDC), Via del Politecnico, 00133 Rome, Italy.
}

Corresponding author email: edoardo.rognini@inaf.it

Keywords: Mercury, BepiColombo, Surface temperature.

BepiColombo is Europe's first mission to Mercury. The spacecraft will arrive at Mercury on December 2025, and magnetic field, magnetosphere, and both internal and external structures will be studied.

A simple thermal model has been derived, with the aim of analyzing the dependence of the temperature on the surface of the planet and in the upper part of the crust on a wide range of assumptions on the soil properties. The code calculates the surface temperature with a one dimensional approach. We assume that heat is transferred only by conduction, and no radioactive heat sources are present; a regolith layer, characterized by a lower density and higher porosity, has been assumed on the surface. The model will be used to test various hypotheses on the physical properties of the surface and upper crust, and to help in interpreting the data from the instruments onboard BepiColombo. A simpler version of the model will be made available to the scientific community with the use of MATISSE [1], the software tool developed at SSDC in ASI.

\section{References:}

[1] Zinzi et al. (2016) - Astronomy \& Computing. 


\title{
First evidence for bright-toned megaripple migration on Mars
}

\author{
Silvestro S.1 ${ }^{2}$, Chojnacki M. ${ }^{3}$, Vaz D.A. ${ }^{4}$, Cardinale M. ${ }^{5}$, Mongelluzzo G. ${ }^{1} \&$ Esposito F. ${ }^{1}$ \\ ${ }^{1}$ INAF Osservatorio Astronomico di Capodimonte, Napoli, Italy. \\ ${ }^{2}$ SETI Institute, Carl Sagan Center, Mountain View, CA, USA. \\ ${ }^{3}$ Lunar and Planetary Lab., U.A., Tucson, AZ, USA. \\ ${ }^{4}$ Centre for Earth and Space Research of the University of Coimbra, Coimbra, Portugal. \\ ${ }^{5}$ DiSPUTer, Univ. G. d'Annunzio, Chieti, Italy.
}

Corresponding author email: simone.silvestro@inaf.it

Keywords: Mars, Aeolian bedforms, Ripples.

The action of the wind has accumulated vast fields of aeolian bedforms on the surface of Mars. Due to the paucity of surface wind measurements, orbital analysis of these features is fundamental to constrain present and past climatic conditions. Along with the dunes, two other types of aeolian bedforms have been identified: 1) dark toned ripples (DTRs) (1-5 m spaced) which overprint the dune's slopes [1] or form vast isolated fields [2-4], and 2) Transverse Aeolian Ridges (TARs), which are bright-toned features 10-100 m spaced and $\sim 1-$ $14 \mathrm{~m}$ tall [5]. Active DTRs found on dune slopes have been interpreted as normal ripples [2,6] or fluid-drag ripples [7], whereas inactive DTRs not associated with dunes have been suggested to be megaripples [3, 4]. In this report, we investigate the nature and activity of 8-18 meter-spaced bright-toned bedforms which have sizes in between DTRs and TARs by using overlapping HiRISE images. The Martian bright-toned bedforms are located behind or in between dunes suggesting a megaripple origin. The megaripples show modifications on a temporal baseline of $\sim 8-10$ Earth years and their activity come as a surprise. Like the active DTRs found on the dune slope, the megaripples can migrate obliquely and seem to be associated with high sand flux dunes. The presence of these active megaripples together with inactive bedforms suggest a complex aeolian scenario characterized by strong winds and bedform induration. The megaripple migration rates and direction can be used to track strong Martian winds and will provide further ground truth for atmospheric models.

\section{References:}

[1] Bridges et al. (2007) - GRL, 34, L23205.

[2] Sullivan et al. (2008) - JGR, 113, E06S07.

[3] Golombek et al. (2010) - JGR, 115, E00F08.

[4] Fenton et al. (2018) - JGR, 123.

[5] Zimbelman et al. (2013) - Aeol. Res., 11.

[6] Kok et al. (2012) - RPP, 75, 106901.

[7] Lapotre et al. (2016) - Sci., 353, 6294. 


\section{Thermal mapping of Io obtained with the JIRAM instrument onboard the Juno mission}

Tosi F. ${ }^{1}$, Mura A. ${ }^{1}$, Adriani A. ${ }^{1}$, Filacchione G. ${ }^{1}$, Sindoni G. ${ }^{2}$, Altieri F. ${ }^{1}$, Grassi D. ${ }^{1}$, Migliorini A. ${ }^{1}$, Moriconi M.L. ${ }^{1}$, Piccioni G. ${ }^{1}$, Plainaki C. ${ }^{1,2}$, Turrini D. ${ }^{1}$, Cicchetti A. ${ }^{1}$, Noschese R. ${ }^{1} \&$ Sordini R. ${ }^{1}$

${ }^{1}$ INAF-IAPS, Via del Fosso del Cavaliere 100, I-00133 Rome, Italy.

${ }^{2}$ Agenzia Spaziale Italiana, Via del Politecnico snc, 00133 Rome, Italy.

Corresponding author email: federico.tosi@,iaps.inaf.it

Keywords: Jupiter, Juno mission, Thermal mapping, JIRAM.

The Jupiter InfraRed Auroral Mapper (JIRAM) onboard the NASA Juno mission [1] is a spectro-imager designed primarily for the study of atmospheric and auroral processes occurring on the planet Jupiter. However, JIRAM has occasionally observed also the Galilean satellites, Jupiter's largest moons. It should be noted that these are not planned observations: they usually occur when these objects randomly enter the field of view of JIRAM.

JIRAM observed the moons both with its double infrared camera ( $\mathrm{L}$ band centered at $3.45 \mu \mathrm{m}$ and $\mathrm{M}$ band centered at $4.78 \mu \mathrm{m}$ ) and with its spectrometer covering the $2-5 \mu \mathrm{m}$ infrared range. In the overall period between 10 July 2016 and 25 May 2018, the Galilean satellites were observed a total of $\sim 4100$ times. Depending on the target and on the specific observation, the spatial resolutions varied between 78 and $2063 \mathrm{~km} / \mathrm{px}$, apparent angular radii ranged between about 1 and 29 JIRAM pixels, and solar phase angles were medium-to-high in general $\left(52^{\circ}-111^{\circ}\right)$. Although the achieved spatial resolutions were not exceptional, the polar orbit of Juno around Jupiter makes it possible to perform observations of the moons at high latitudes, which are inaccessible to Earth-based or space-based telescopes.

Compared to the icy Galilean satellites, the case of Io is intriguing with regard to thermal mapping: the surface of this satellite is dotted with a multitude of active volcanic centers, which make JIRAM a key instrument for the thermal monitoring of Io's surface both at the local scale (where the sulfur-rich lavas erupted by volcanoes can be hotter than $1000 \mathrm{~K}$ ) and at regional/hemispheric scale. JIRAM observations of Io are definitely more frequent than planned Earth-based, infrared telescopic observations of Io.

\section{References:}

[1] Adriani et al. (2017) - JIRAM, the Jovian Infrared Auroral Mapper. Space Sci. Rev. 213 (1-4), 393-446. 


\section{Spectral analysis of the Apollo basin on the Moon}

Zambon F. ${ }^{1}$, Carli C. ${ }^{1}$, van der Bogert C.H. ${ }^{2}$, Hiesinger H. ${ }^{2}$, Altieri F. ${ }^{1}$, Massironi M..$^{3,4} \&$ Capaccioni F. ${ }^{1}$

${ }^{1}$ INAF-Istituto di Astrofisica e Planetologia Spaziali, Via del Fosso del Cavaliere, 100, I-00133 Rome.

${ }^{2}$ Institut für Planetologie, Westfälische Wilhelms Universität, Wilhelm-Klemm-Str. 10, 48149 Münster, Germany.

${ }^{3}$ Dipartimento di Geoscienze, Padova University, Padova, Italy.

${ }^{4}$ INAF - Osservatorio Astronomico di Padova.

Corresponding author email: francesca.zambon@inaf.it

Keywords: Apollo basin, impact crater, South-Pole Aitken, Moon.

Studies, e.g. [1, 2], reveal that the South-Pole Aitken (SPA) basin is unique, particularly because it is the deepest basin on the Moon and might be expected to expose the lunar mantle [3]. The innermost part of SPA is rich in high Ca-pyroxenes, except for some central crater peaks, where low Ca-pyroxenes dominate. No extensive olivine-rich areas are observed, implying a mantle rich in low Ca-pyroxenes instead of olivine [1, 3, 4]. Furthermore, thorium anomalies have been discovered in two craters within Aitken basin [5]. Here, we focus on the spectral analysis of one of the most interesting impact craters inside SPA, the Apollo basin. Recently, [6] published a detailed geological map of the area surrounding Apollo basin $\left(35.69^{\circ} \mathrm{S}, 151.48^{\circ} \mathrm{W}\right.$; D $\left.524 \mathrm{~km}\right)$, using morphology, stratigraphy, crater size-frequency distribution measurements, and Clementine spectral data to define the unit. Our work explores data from the M3 imaging spectrometer onboard Chandrayaan-1 [7] for this region. These analyses are part of the PLANMAP project, and the integration of the spectroscopical information with the results from [6] will permit to produce highly informative geological maps of the Moon [8].

This work is funded by the European Union's Horizon 2020 research grant agreement No 776276PLANMAP.

\section{References:}

[1] Ohtake et al. (2014) - GRL.

[2] Moriarty et al. (2018) - JGR.

[3] Dhingra (2018) - Geosciences.

[4] Melosh et al. (2017) - Geology.

[5] Lawrence et al. (2000) - JGR.

[6] Ivanov (2018) - JGR.

[7] Pieters et al. (2009) - Current Science.

[8] https://www.planmap.eu/ 


\title{
Spectral variations across H-05 Hokusai quadrangle on Mercury: a connection between spectral properties and morpho-stratigraphic units
}

\author{
Zambon F. ${ }^{1}$, Carli C. ${ }^{1}$, Wright J. ${ }^{2}$, Altieri F. ${ }^{1}$, Giacomini L. ${ }^{1}$, Massironi M. ${ }^{3,4}$, Capaccioni F. ${ }^{1}$, Rothery D.A. ${ }^{2}$, \\ Cremonese G. ${ }^{4} \&$ the PLANMAP Team \\ ${ }^{1}$ INAF-Istituto di Astrofisica e Planetologia Spaziali, Via del Fosso del Cavaliere, 100, I-00133 Rome. \\ ${ }^{2}$ School of Physical Sciences, Open University, Milton Keynes, UK. \\ ${ }^{3}$ Dipartimento di Geoscienze, Padova University, Padova, Italy. \\ ${ }^{4}$ INAF - Osservatorio Astronomico di Padova.
}

\section{Corresponding author email: francesca.zambon@inaf.it}

Keywords: Mercury, H-05 Hokusai quadrangle, Morpho-stratigraphy.

H05 Hokusai $\left(22.5^{\circ} \mathrm{N}-65^{\circ} \mathrm{N}, 0^{\circ}-90^{\circ} \mathrm{E}\right)$ is one of the northern quadrangles of Mercury $[1,2]$. It is characterized by different features and includes several morpho-stratigraphic units. Smooth plains is the most widespread, covering $\sim 43 \%$ of the entire quadrangle, followed by fresh crater material $(\sim 17 \%)$ and intercrater plains $(\sim 10 \%)$. The remaining part of the quadrangle contains intermediate plains $(7 \%)$, degraded crater material $(9 \%)$ and heavily degraded material $(8 \%)$ [1]. Other morpho-stratigraphic units, such as smooth and hummocky crater floor material, account for a few percent [1]. Furthermore, this quadrangle includes part of the Hokusai ray system, the largest example on Mercury, and several pyroclastic deposits, including Nathair Facula, one of the highest-reflectance features observed on the planet, located at $480 \mathrm{~km}$ NE of Rachmaninoff crater [3, 4]. Spectral analysis based on specific spectral parameters represents a suitable approach to investigate compositional variations. We observed different spectral behavior among large parts of the morpho-stratigraphic units and surface features mapped by [1]. In particular, the units that show the highest spectral variability are the intermediate plains, smooth plains and fresh crater material, together with pyroclastic deposits and crater rays. Such a result indicates spectral variation related to the different units with implications for the geological processes on Mercury's surface.

This work is funded by the European Union's Horizon 2020 research grant agreement No 776276PLANMAP and by the Italian Space Agency (ASI) within the SIMBIO-SYS project (ASI-INAF agreement 2017-47-H.0).

\section{References:}

[1] Wright et al. (2018) - LPSC 2018.

[2] https://planetarynames.wr.usgs.gov/Page/mercuryQuadMap

[3] Rothery et al. (2018) - Oxford Research Encyclopedia of Planetary Science. Ed.

[4] Procter L. et al. (2010) - Science 329. 


\title{
MATISSE 2.0: towards a hub for the planetary science community
}

\author{
Zinzi A. ${ }^{1}$, Giardino M. ${ }^{1}$, Sindoni G. ${ }^{2}$, Ammannito E. ${ }^{2}$, Perozzi E. ${ }^{2}$, Plainaki C. ${ }^{2}$, Capria M.T. ${ }^{3}$ \& Palomba E. \\ ${ }^{1}$ Space Science Data Center - ASI. \\ ${ }^{2}$ Agenzia Spaziale Italiana. \\ ${ }^{3}$ INAF-IAPS.
}

Corresponding author email: angelo.zinzi@,ssdc.asi.it

Keywords: MATISSE, Dataset, SSDC.

After almost 6 years, MATISSE [1] is in a deep updating phase, aimed at improving its interface and services and likely to be completed in some months. Among the main differences with the old version, it is worthy to note that MATISSE 2.0, using a servlet-based configuration, will be available both from the web-interface and from command-line, thus helping the user to perform advanced and iterated operations. The 3D capabilities of the present version will be maintained, and its interactive capabilities for the $2 \mathrm{D}$ will be improved using the Planetary FITS standard [2]. The main goal of MATISSE 2.0 is to make the fusion between different datasets easier and more scientific valuable, indifferently using data stored in local SSDC repository or accessible by remote through international standards. The tool will be also developed to allow access to modelled datasets useful for comparison purposes. These tasks will be pursued by means of collaborations, both inside and outside ASI. Regularly submitted inputs from the recently acquired ASI personnel with planetary sciences expertise, covering several fields, from small bodies to space weather, passing through atmospheric physics and advanced ICT technologies, should guarantee major advances and an extremely solid scientific base. A series of new collaborations with international scientific research groups involved in different missions and activities (i.e., ExoMars, Juno, BepiColombo, VESPA, Open Planetary, NEO hazard and planetary defense), will make the tool even more versatile, further opening it to a wider community, to fully exploit the possibilities offered. Thanks to these efforts, MATISSE 2.0 could become a hub through which a wide set of planetary exploration datasets could be accessed and analyzed by all the scientific community, thus greatly improving scientific capabilities of every mission included in the project.

\section{References:}

[1] Zinzi et al. (2016) - Astronomy \& Computing.

[2] Marmo et al. (2018) - Earth and Space Science. 


\section{DYNAMICS OF CELESTIAL BODIES, EITHER NATURAL OR MAN-MADE}




\title{
Toward a physical characterization of the Soviet/Russian constellation of Molniya satellites
}

\author{
Buzzoni A. ${ }^{1}$, Guichard J. ${ }^{2}$, Matassoni F. ${ }^{3}$, Figer A. ${ }^{4}$, Altavilla G. ${ }^{5} \&$ Galleti S. ${ }^{1}$ \\ ${ }^{1}$ INAF - OAS Bologna. \\ ${ }^{2}$ INAOE, Puebla, Mexico. \\ ${ }^{3}$ DIFA UniBo, Bologna. \\ ${ }^{4}$ GEOS, Paris, France. \\ ${ }^{5}$ INAF - OAR Roma.
}

Corresponding author email: alberto.buzzoni@inaf.it

Keywords: space debris, artificial satellites, spectrophotometry.

We report on the extended observing campaign, carried out at Mexican and Italian telescopes between 2014/17, to characterize from the dynamical and spectrophotometric point of view the whole constellation of 43 surviving Soviet/Russian spacecraft Molniya, currently in HEO orbit [1]. In the historical context, the Molniya project stands out as the first innovative concept of "satellite constellation" to deliver advanced communication services in terms of coordinated action of a set of satellites. The full constellation now consists of dead payloads orbiting under uncontrolled dynamical regime. Through a combined observation of the spacecraft population (and taking advantage that all satellites sport a similar structure and bus configuration) we especially addressed the impact of ageing effects on the colour and reflectance properties, orbital parameters and spin rates as affected by the magnetospheric drag. The distinctive properties of colours and absolute magnitude with orbital phase angle for the whole satellite population have also been assessed by comparing with natural (NEO, the Moon etc.[2]) and artificial (GEO) reference templates [3] [4][5]. Orbit evolution (along four decades, for the oldest satellites in our sample), has also been an issue, by studying the influence of luni-solar resonance effects [6][7], through JSPOC historical TLE information. The work is an outstanding propaedeutic effort in the framework of the incepting SSA/SST activity in Italy, as coordinated by OCIS, aimed at fully characterizing the orbiting population of artificial space debris.

\section{References:}

[1] Fortescue \& Stark (1995) - Spacecraft system engineering (Wiley \& Sons).

[2] Buratti et al. (2011) - JGRE, 116, E00G03.

[3] Brambilla (2011) - MSc thesis, DIFA UniBO.

[4] Matassoni (2017) - MSc thesis, DIFA UniBO.

[5] Cardona (2012) - MSc thesis, Fac. Ingegneria Aerospaziale, UniBo.

[6] Rossi (2008) - CEMDA 100, 267.

[7] Daquin et al. (2016) - CEMDA, 124, 335. 


\title{
The Fly Eye Telescope is growing up - Development status and first lights from Sky
}

\author{
Cibin L. ${ }^{1}$, Chiarini M. ${ }^{1}$, Bernardi F. ${ }^{2}$, Ragazzoni R. ${ }^{3}$, Salinari P. ${ }^{4}$, Sessler G. ${ }^{5}$ \& Kugel U. ${ }^{5}$ \\ ${ }^{1}$ OHB Italia Spa, Milano, Italy - (CGS). \\ ${ }^{2}$ Space Dynamics Services, Navacchio di Cascina (PI), Italy - (SpaceDyS). \\ ${ }^{3}$ Istituto Nazionale di Astrofisica - Osservatorio Astronomico di Padova, Padova, Italy - (INAF). \\ ${ }^{4}$ Istituto Nazionale di Astrofisica - Osservatorio Astrofisico di Arcetri, Firenze, Italy - (INAF). \\ ${ }^{5}$ European Space Agency, Robert-Bosch-Str. 5 - 64293 Darmstadt (Germany).
}

Corresponding author email: 1cibin@cgspace.it

Keywords: Near-Earth-Asteroids, telescopes, survey.

The NEOSTED ESA program has led to the integration of a first FlyEye Telescope Prototype, provided with half Field of View (22 sq. degs. over full 44) optics. A dedicated GSTP program - ASTROCAD - has started to design and develop the astronomic Cameras, necessary to populate the Telescope Focal Plane with astronomic grade CCD sensors.

The procurement of the remaining optics, necessary for the completion of the full FoV, has been launched. Advanced optical alignment methods have been implemented based on Newton's rings technique, to allow the overall optics progressive integration exploiting the modular architecture of the FlyEye. First on Sky test campaign has led to encouraging results, in line with Telescope design. The Astronomical Site for the Telescope deployment has been selected and Infrastructure procurement has been launched. We present here the status of the program and the preliminary results obtained with on field observations. 


\section{Astronomical seeing evaluation for NEO surveys at Monte Mufara and at the ASI-CGS in Matera}

Di Cecco A. ${ }^{1}$, Perozzi E. ${ }^{1}$, Guarcello M. ${ }^{2}$, Iacovone D. ${ }^{3}$, Bianco G. ${ }^{1}$, Buzzoni A. ${ }^{4}$, Di Paola A. ${ }^{5}$, Falvella M.C. ${ }^{1}$, Fierro D. ${ }^{6}$, Marzo C. ${ }^{1} \&$ Micela G. ${ }^{2}$

${ }^{1}$ Agenzia Spaziale Italiana (ASI).

${ }^{2}$ INAF-OAPa.

${ }^{3}$ E-Geos.

${ }^{4}$ INAF OAS.

${ }^{5}$ INAF-OAR.

${ }^{6}$ INAF.

Corresponding author email: alessandra.dicecco@yahoo.it

Keywords: Near-Earth-Asteroids, Fly-Eye telescopes, survey.

The so called "Fly-Eye" telescope of the ESA SSA Programme will be the main European facility devoted to the NEO discovery. When it will be operational will scan the night-sky in robotic mode, collecting mosaic images based on 16 CCD sub-images, with a total Field-of-View of $6.7 \times 6.7$ squared degree and resolution of $1.5 \mathrm{arcsec} / \mathrm{pixel}$. Recently, the National site of Monte Mufara in Sicily has been selected for hosting this telescope and the installation is planned by the end of 2019. This selection has been supported by several efforts made by a strong scientific and technical collaboration between ASI and INAF. In particular, one of the specific requirements for hosting this telescope concerns the evaluation of the astronomical seeing value, which should be comparable to the pixel scale. To this purpose, a seeing monitor has been installed on the top of the Monte Mufara, collecting data for more than one year. Moreover, a plan for the telescope commissioning and science verification phases is going to be defined. A preliminary plan proposes to conduct these phases in a comfortable and safety place (before the Monte Mufara installation), such as the Center for Space Geodesy (CGS) of ASI in Matera. Moving the telescope in the CGS for a while, will permit to achieve accurate observational campaings, allowing to conduct preliminary tests for the pointing system, data acquisition system, software products generation, etc. To this purpose, a second seeing monitor has been installed in the outer area of the CGS.

We present the datasets and the analysis of the astronomical seeing measurements collected during the last year for both the Monte Mufara and the CGS observational sites. We discuss the strategy adopted to evaluate the astronomical seeing, starting by the raw data and signal-to-noise considerations. We discuss the possible occurrence of seasonal variation. Our results suggest that the seeing values are compliant to the requirements for the "Fly-Eye" preliminary test phases and installation. Moreover, we discuss the observational limits that may affect the observations in terms of the local sky brightness and cloud-coverage. 


\title{
Numerical simulations of space debris populations with symplectic integrators
}

\author{
Di Cintio P. ${ }^{1}$, Rossi A. ${ }^{1} \&$ Valsecchi G.B. ${ }^{1,2}$ \\ ${ }^{1}$ IFAC-CNR. \\ ${ }^{2}$ IAPS-INAF.
}

Corresponding author email: pdicintio@gmail.com

Keywords: space debris, orbits, numerical integrators, population modelling.

In the study of the long-term evolution of the space debris population it is of paramount importance to be able to achieve a good compromise between accuracy and computational speed, in order to propagate the orbit of thousands of objects at the same time.

We present a novel implementation of a symplectic orbital propagator in Cartesian coordinates for the dynamics of space debris from the Low to High Earth orbit range. Test simulations including luni-solar perturbations suggest that our optimized numerical code can attain a significant reduction in computational times with respect to previous orbit propagators based on averaged dynamics in orbital elements.

Moreover, we show preliminarily results for case studies where the effects of non-gravitational perturbations such as solar radiation pressure and atmospheric drag, often not accounted for in symplectic integrators, are considered.

Finally, the comparison between the results of a few long term evolution scenarios simulated with the SDM model, using both the original propagator (working on averaged dynamics in orbital elements) and the new one, are presented. 


\title{
Physical Properties of Near Earth Objects: the legacy of the EU H2020 programme NEOShield-2
}

\author{
Dotto E. ${ }^{1}$, Mazzotta Epifani E. ${ }^{1}$, Perna D. ${ }^{1}$, Ieva S. ${ }^{1}$, Perozzi E. ${ }^{2}$, Lazzarin M. ${ }^{3} \&$ Bertini I. ${ }^{3}$ \\ ${ }^{1}$ INAF-Osservatorio Astronomico di Roma, Via Frascati 33, 00078 Monte Porzio Catone (Roma). \\ ${ }^{2}$ ASI-Agenzia Spaziale Italiana, Via del Politecnico, 00133 Roma. \\ ${ }^{3}$ Dipartimento di Fisica e Astronomia “G. Galilei”, Università di Padova, Vicolo dell’ Osservatorio 3, 35122 Padova. \\ Corresponding author email: elisabetta.dotto@inaf.it
}

Keywords: Near Earth Asteroids, physical properties of asteroids, spectrophotometry.

In the last years the research about Near Earth Objects (NEOs) has been a major topic in planetary science, also in view of the potential hazard some of them pose to human beings and, more in general, to life on our planet.

Moreover, the physical characterization of NEOs allows us to put constraints on the material in the protoplanetary nebula at different solar distances and can give us insights into the early processes that governed the formation and the evolution of planets, including the delivery of water and organics to Earth.

The European Commission approved and financed the NEOShield-2 (2015-2017) in the framework of the Horizon 2020 programme, with the aims i) to study specific technologies and instruments to conduct close approach missions to NEOs or to undertake mitigation demonstration, and ii) to acquire in-depth information of physical properties of the population of NEOs between 50 and $300 \mathrm{~m}$, in order to design mitigation missions and assess the consequences of an impact on Earth.

In the framework of this programme we observed and characterized more a large number of NEOs. The obtained results and the legacy of the NEOShield-2 programme will be presented and discussed. 


\title{
Orbital evolution of the Galilean satellites driven by tidal dissipation
}

\author{
Lari G. ${ }^{1}$, Saillenfest M. ${ }^{2} \&$ Fenucci M. ${ }^{1}$ \\ ${ }^{1}$ Department of Mathematics, University of Pisa. \\ ${ }^{2}$ IMCCE, Observatoire de Paris
}

Corresponding author email: 1ari@mail.dm.unipi.it

Keywords: orbits, tides, Galilean satellites, orbital evolution.

The Galilean Satellites are the four biggest satellites of Jupiter; impressive geophysical phenomena take place on and under their surface, such as volcanic eruptions and preservation of liquid water oceans, both due to the tidal forces. Moreover, the motion of the three inner moons (Io, Europa and Ganymede) is characterized by a three-body mean motion resonance, called Laplace resonance. The construction of a long-term dynamical model is not straightforward, as shown in [3], because of the presence of resonant angles, which increases considerably the number of terms in the expansion of the perturbations. Using a new semi-analytical model presented in [2], we investigate the secular evolution of the system under the action of the tidal dissipation. In particular, considering the values of the dissipative parameters of Io and Jupiter obtained in [1], we investigate the possible capture in resonance of Callisto.

\section{References:}

[1] Lainey et al. (2009) - Nature.

[2] Lari (2018) - CMDA.

[3] Malhotra (1991) - Icarus. 


\title{
State of the art of the measurement of the Lense-Thirring effect after a century from its formulation
}

\author{
Lucchesi D.M. ${ }^{1,2,3}$, Anselmo L. ${ }^{2}$, Bassan M. ${ }^{3,4}$, Magnafico C. ${ }^{1,3}$, Pardini C. ${ }^{2}$, Peron R. ${ }^{1,3}$, \\ Pucacco G. ${ }^{3,4}$ \& Visco M. ${ }^{1,3}$ \\ ${ }^{1}$ Istituto di Astrofisica e Planetologia Spaziali (IAPS/INAF), Via Fosso del Cavaliere, 100, Tor Vergata, Roma. \\ ${ }^{2}$ Istituto di Scienza e Tecnologia della Informazione (ISTI/CNR), Via Moruzzi, 1, San Cataldo, Pisa. \\ ${ }^{3}$ Istituto Nazionale di Fisica Nucleare (INFN), Via della Ricerca Scientifica, 1, Tor Vergata, Roma. \\ ${ }^{4}$ Dipartimento di Fisica, Università di Tor Vergata, Via della Ricerca Scientifica, 1, Tor Vergata, Roma.
}

Corresponding author email: david.lucchesi@inaf.it

Keywords: general relativity, Lense-Thirring effect, gravity field.

The motion of passive laser-ranged satellites along nearly geodesics of spacetime may be a posteriori reconstructed through a careful modelling of the main non-gravitational perturbations (NGP) acting on their surface. The Laser Ranged Satellites Experiment (LARASE) [1] aims to test the predictions of Einstein's theory of general relativity (GR) in its weak-field and slow-motion limit with respect to those of alternative theories of gravitation. An effort of the LARASE activities is to strongly improve the modelling of the NGP on the two LAGEOS and LARES satellites in such a way to further improve the orbit determination of these satellites and extract from their orbital residuals the sough for relativistic effects. Among some of the recent activities of LARASE regarding the NGP, we focus upon the development of a new model for the spin evolution of the satellites [2] and that for the subtle effects on their orbit produced by the thermal thrust perturbations. Concerning the gravitational perturbations, we discuss our improvements in the knowledge of the even zonal harmonics coefficients based on a re-analysis of GRACE data. With all this information we provide our results for a new precise and accurate measurement of the Earth's gravitomagnetic effect based on the analysis of the Lense-Thirring precession on the combined orbits of the LAGEOS, LAGEOS II and LARES satellites. Gravitomagnetism plays a special role in Einstein's geometrodynamics, it describes the curvature of spacetime produced by mass-currents, with important consequences in the astrophysics of high-energy phenomena as well as for its possible cosmological consequences related to Mach's principle.

\section{References:}

[1] Lucchesi et al. (2015) - Quantum Grav. 32, 155012. DOI 10.1088/0264-9381/32/15/155012.

[2] Visco \& Lucchesi (2018) - Comprehensive model for the spin evolution of the LAGEOS and LARES satellites, Phys. Rev. D98(4), 044034. DOI 10.1103/PhysRevD.98.044034. 


\section{LICIACube: the Light Italian Cubesat for Imaging of Asteroid in support to the NASA DART mission}

Mazzotta Epifani E. ${ }^{1}$ on behalf of the LICIACube team*

${ }^{1}$ INAF-Osservatorio Astronomico di Roma, Via Frascati 33, 00078 Monte Porzio Catone (Roma).

Corresponding author email: elena.mazzottaepifani@inaf.it

Keywords: Near Earth Asteroids, deflection, impacts, orbit determination.

The NASA Double Asteroid Redirection Test (DART) mission will be the first demonstration of the kinetic impact technique to change the orbital energy of an asteroid in space. It is a planetary defence-driven test of one of the technologies for preventing the Earth impact of hazardous asteroids: the kinetic impactor. DART's primary objective is to demonstrate a kinetic impact on a small asteroid, the binary near-Earth asteroid (65803) Didymos. While Didymos' primary body is approximately 800 meters across, its secondary body (or "moonlet") has a 150-meter size, which is a typical size of asteroids that could pose a common hazard to Earth.

The Light Italian Cubesat for Imaging of Asteroids (LICIACube) is the Italian contribution to the mission, coordinated by the Agenzia Spaziale Italiana (ASI). It is a project gathering together national research centres, academies, and private sector acting in the space domain. The consortium focuses on finalizing a challenging mission to document the DART spacecraft most relevant phase: the impact. The scientific requirements of the cubesat mission deal with the observation of the slow plume ejecta evolution just after the DART impact occurrence, the investigation of the plume density structure, and, possibly, the observation of the non-impact moonlet hemisphere.

In this talk we present the expected scientific performances of LICIACube, and the science that will be performed at the target.

* The LICIACube team is composed by:

INAF: E. Dotto, E. Mazzotta Epifani, D. Perna, S. Ieva (INAF-OAR), V. Della Corte, S. Ivanovski (INAF-IAPS), J.R. Brucato (INAF-OAA), G. Cremonese, A. Lucchetti (INAF-OAPd).

Politecnico di Milano: M. Lavagna, A. Capannolo, V. Pesce.

Università Parthenope (Napoli): P. Palumbo.

Università di Bologna: P. Tortora, D. Modenini, M. Zannoni, I. Gai.

ASI: S. Pirrotta, E. Ammannito, M. Amoroso, S. Pizzurro.

Argotec (Torino): V. Di Tana, S. Simonetti, B. Cotugno. 


\title{
The new wide-field instruments installed on Madonie Regional Natural Park to study and monitor fast moving and transient phenomena
}

\author{
Nastasi A. ${ }^{1}$, Spanò P. ${ }^{2}$, Masiero S. ${ }^{1} \&$ Ziino L. ${ }^{1}$ \\ ${ }^{1}$ Fondazione GAL Hassin - Centro Internazionale per le Scienze Astronomiche, Isnello (PA). \\ ${ }^{2}$ Officina Stellare, Thiene (VI).
}

Corresponding author email: alessandro.nastasi@galhassin.it

Keywords: Near-Earth-Asteroids, Wide-field Mufara Telescopes, survey.

In the new era of time-domain astronomy, it is crucial to monitor the night sky via instruments able to spot and study potentially faint and short-lived transient events, associated to objects varying in luminosity and/ or position. Wide-field and fast telescopes enable to cover the whole sky every few days, observing down to extremely faint sources, and for this reason they represent the best suited class of instruments for this purpose. The Wide-field Mufara Telescope (WMT) is a 1-meter f2.1 remotely controlled telescope which is being built on Mount Mufara (1865 m), in Sicily. It will be fully operational in 2019. This new instrument, with a field of view (FoV) of 6 square degrees, will represent a crucial facility to monitor and identify new potentially hazardous objects (PHOs) and their trajectories, and it will complement the activities of the largest telescopes which operate at other wavelength windows, in the framework of the multi-messenger astrophysics. Alongside of the WMT, the GAL Hassin Robotic Telescope (GRT) is another wide-field (FoV of 4 square degrees), fast (f3.8) telescope with 400mm aperture installed in the same geographical area (within the GAL Hassin astronomical center) and fully operational since 2016. The GRT is already extensively used to confirm supernovae detections, to characterize (intrinsically and not) variable stars and to monitor the orbits of known PHOs. Both WMT and GRT are located in the Madonie Park, one of the highest regions of Sicily, which has superb astronomical conditions with very limited light pollution, more than 200 clear nights per year and seeing conditions down to 1 " for several hours per night. In addition, the relatively low latitude $38^{\circ}$ makes this region the best place in Italy from where to observe the interesting events occurring close to the center of our Galaxy. In my talk I will detail the current and future goals which can be achieved with the upcoming WMT and the already available GRT telescope. 


\section{The "Near-Earth Space Trekker" (NEST) multi-asteroid mission concept}

Perna D.

INAF-OAR and the NEST team

Corresponding author email: davide.perna@inaf.it

Keywords: Near-Earth-Asteroids, ESA, space mission.

The "Near-Earth Space Trekker" (NEST) mission concept has been proposed by an Italian-French-Swiss consortium in response to the ESA call for a 'Fast' mission opportunity. NEST has been selected in November 2018 with a few other proposals for the Phase 2 evaluation, which will lead to the selection in mid-2019 of one mission concept for the following study phases. The mission scenario and the scientific objectives will be presented and discussed. 


\title{
The BepiColombo ISA accelerometer: flying to Mercury
}

\author{
Santoli F., Iafolla V., Fiorenza E., Lefevre C., Lucchesi D.M., Lucente M., Magnafico C. \& Peron R.
}

IAPS-INAF.

Corresponding author email: roberto.peron@inaf.it

Keywords: Mercury, gravity field, rotation, internal structure, general relativity.

The BepiColombo Italian Spring Accelerometer (ISA) is a component of the dedicated suite of instruments which will perform the so-called Radio Science Experiments in order to study gravitational field, internal structure and rotational state of the planet Mercury, and to verify important predictions of the general theory of relativity. Its main role is the measurement of non-gravitational accelerations acting on the Mercury Planetary Orbiter spacecraft, thereby enabling to work on a virtually drag-free satellite. After many years of development, BepiColombo has been successfully launched from the Kourou Guiana Space Centre on 20 October 2018. A first slot of commissioning activities has been already performed by ISA Team together with ESA colleagues, confirming the health of the instrument; other testing periods will follow in the near future. Here the concept, design and development of the instrument are briefly reviewed, along with its main scientific products and data processing procedures. Special emphasis is given to the results of commissioning and to the operations foreseen for the first part of the cruise phase. 


\title{
A long tradition of orbit determination in Pisa: the Juno mission to Jupiter
}

\author{
Serra D. ${ }^{1}$, Lari G. ${ }^{2}$, Tommei G. ${ }^{2} \&$ Milani A. ${ }^{2}$ \\ ${ }^{1}$ University of Pisa; \\ ${ }^{2}$ University of Pisa
}

Corresponding author email: daniele.serra@dm.unipi.it

Keywords: orbit determination, Jupiter, gravity field, internal structure.

Since its insertion in a Jupiter-centric orbit in 2016, the Juno spacecraft has been exploring the interior, the atmosphere and the magnetosphere of the largest planet of our Solar System. The gravity field, described by the coefficients of its spherical harmonics expansion, is a fundamental element for discriminating amongst various models of Jupiter's interior structure. Thanks to an on-board Ka-band $(\sim 32.5-34 \mathrm{GHz})$ frequency translator, it is possible to measure very accurately the spacecraft range-rate, namely at the level of a few microns per second over $1000 \mathrm{~s}$ integration time. After appropriate pre-processing finalized at reducing the data noise, the Doppler data are analyzed with specific software in order to reconstruct the orbit of the spacecraft as well as to determine a set of parameters of interest, including the spherical harmonics coefficients of Jupiter's gravity field. The most up-to-date solution of the gravity field of Jupiter, from range-rate data collected during the first two Juno Gravity orbits, was presented in [1]. This gravity solution was obtained using JPL's software MONTE and a filter developed at Sapienza University of Rome. In this work we present the solution obtained by processing the same tracking data with the ORBIT14 orbit determination software, developed at the University of Pisa, Italy, under a contract with the Italian Space Agency. This code is designed specifically for the radioscience experiments of the NASA's Juno and ESA/JAXA's BepiColombo missions. The two solutions turned out to be compatible within the formal accuracy, thus confirming the discoveries regarding Jupiter's interior inferred from [1] and described in [2] and [3].

\section{References:}

[1] Iess et al. (2018) - Nature.

[2] Kaspi et al. (2018) - Nature.

[3] Guillot et al. (2018) - Nature. 


\title{
Impact Monitoring of Near-earth Objects: past, present and future challenges
}

\author{
Tommei G. \\ Dipartimento di Matematica - Università di Pisa
}

Corresponding author email: giacomo.tommei@unipi.it

Keywords: Near Earth Asteroids, orbits, impacts, orbit determination.

An asteroid just been discovered has a strongly undetermined orbit, being weakly constrained by the few available astrometric observations, and there is a set of possible orbits, all compatible with the observations, forming a Confidence Region (CR) in the 6-dimensional orbital elements space. The goal of Impact Monitoring (IM) is to understand whether the CR contains subsets of initial conditions leading to a collision with the Earth in the future (Virtual Impactors, VIs) and to estimate the Impact Probability (IP). Once defined the CR, the crucial steps are the sampling of the uncertainty region, the propagation of the so called Virtual Asteroids (VAs) searching for VIs and the computation of IP. Two automatic systems ([1]), CLOMON2 (at University of Pisa/SpaceDyS/ESA-NEOCC) and Sentry (at JPL/NASA), have been developed for this purpose. Both generate VAs by applying a 1-dimensional sampling of the CR based upon the Line Of Variations (LOV), that is a differentiable curve representing a kind of spine of the uncertainty region. The LOV method is very useful when the CR is elongated and thin, but this is not the case when the observed arc is very short: the uncertainty results to be wide in at least two directions and the LOV is not a reliable representative of the entire region. Unfortunately, this is precisely the case of very small asteroids observed only shortly before a close approach or an impact with the Earth (imminent impactors). The problem has been faced recently and two systems were developed, SCOUT (at JPL/NASA, [2]) and NEOScan (at University of Pisa/SpaceDyS, [3]). In this talk I will try to retrace the main steps of the IM history, focusing on the main algorithms and results, and trying to explore what will be the future developments in this field.

\section{References:}

[1] Milani et al. (2005) - Icarus.

[2] Farnocchia et al. (2015) - Icarus.

[3] Spoto et al. (2018) - A\&A. 


\section{Comparing NEA surveys}

Valsecchi G.B.

IAPS-INAF e IFAC-CNR.

Corresponding author email: giovanni.valsecchi@iaps.inaf.it

Keywords: Near Earth Asteroids, surveys, orbits.

The vast majority of the known NEAs have been discovered by a small number of very capable NEA surveys.

A natural question to ask is whether the most efficient surveys discover similar proportions of the various types of NEAs. Similarly, one can ask whether the differences in discovery efficiency of the NEA types can be meaningfully linked to differences in the hardware and in the observational strategies of the surveys.

We use the discovery statistics available at the MPC to address these questions. 


\section{MINOR BODIES}




\section{Opposition effect on Ceres from VIR-Dawn observations}

Ciarniello M. ${ }^{1}$, De Sanctis M.C. ${ }^{1}$, Ammannito E. ${ }^{2}$, Raponi A. ${ }^{1}$, Carrozzo F.G. ${ }^{1}$, Longobardo A. ${ }^{1}$, Tosi F. ${ }^{1}$, Rognini E. ${ }^{1}$, Zambon F. ${ }^{1}$, Schröder S. ${ }^{3}$, Raymond C.A. ${ }^{4}$, Russell C.T. ${ }^{5} \&$ the VIR team.

${ }^{1}$ IAPS-INAF, Rome, Italy.

${ }^{2}$ ASI, Rome, Italy.

${ }^{3}$ DLR, Institute of Planetary Research, Berlin, Germany.

${ }^{4}$ JPL, California Institute of Technology, Pasadena, CA, USA, 5 UCLA, Earth Planetary and Space Sciences, Los Angeles, CA, USA

Corresponding author email: mauro.ciarniello@inaf.it

Keywords: Ceres, Dawn mission, reflectance properties.

The opposition effect (OE) is a surge of reflectance commonly detected on the surface of atmosphereless bodies [1], when observed at low phase angles. The acquisitions obtained by the VIR mapping spectrometer [2] (0.25-5.1 $\mu \mathrm{m}$ spectral interval) on-board the Dawn mission, during the Extended Mapping Orbit 4 mission phase, allowed the observation of Ceres' surface down to $\sim 0^{\circ}$ phase angle, permitting the characterization of the OE. This set of measurements complements previous observations performed during Dawn mission at Ceres, that covered the $7^{\circ}-132^{\circ}$ phase angle range, and allows to extend the analysis of the Ceres's spectrophotometric properties reported in [3]. The investigation of Ceres' reflectance curve at small phase angles does not show clear evidence of wavelength dependence across the VIS-IR spectral range, pointing to a minor contribution of Coherent Backscattering [1] to the OE. Under the assumption that OE is driven by Shadow Hiding [1], we apply the Hapke's photometric model [1] to VIR data across the investigated spectral interval, determining the corresponding set of parameters for Ceres and deriving the geometric albedo $(0.098 \pm 0.007$ at $0.55 \mu \mathrm{m})$. In addition, we compare this set of observations with Monte Carlo ray-tracing simulations [4], providing an estimate of the surface porosity. When compared to other asteroids, the opposition effect properties of Ceres are typical of low-albedo objects and compatible with the C-type class.

\section{Acknowledgements:}

This work is supported by the Italian Space Agency (ASI, ASI-INAF n. I/004/12/1) and NASA. Enabling contributions from the Dawn Instrument, Operations, and Science Teams are gratefully acknowledged.

\section{References:}

[1] Hapke (2012) - Theory of reflectance and emittance spectroscopy. Cambridge University Press.

[2] De Sanctis et al. (2011) - Space Sci. Rev., 163, 329.

[3] Ciarniello et al. (2017) - A\&A 598, A130.

[4] Ciarniello et al. (2014) - Icarus 237, 293-305. 


\title{
The seasonal cycle of water ice at the surface of $67 \mathrm{P} / \mathrm{Churyumov}-G e r a s i m e n k o$ as observed by VIRTIS-Rosetta
}

\author{
Ciarniello M. ${ }^{1}$, Capaccioni F. ${ }^{1}$, Filacchione G. ${ }^{1}$, De Sanctis M.C. ${ }^{1}$, Capria M.T. ${ }^{1}$, Raponi A. ${ }^{1}$, \\ Formisano M. ${ }^{1}$, Tosi F. ${ }^{1}$, Longobardo A. ${ }^{1}$, Rinaldi G. ${ }^{1}$, Erard S. ${ }^{2}$, Bockelee-Morvan D. ${ }^{2}$, Leyrat C. ${ }^{2}$, \\ Arnold G. ${ }^{3}$, Barucci M.A. ${ }^{2}$, Quirico E. ${ }^{4}$, Fornasier S. ${ }^{2}$, Kappel D. ${ }^{3}$, Rousseau B. ${ }^{1}$, \\ Mottola S. ${ }^{3} \&$ the Rosetta/VIRTIS Team \\ ${ }^{1}$ IAPS-INAF Rome, Italy. \\ ${ }^{2}$ LESIA, Observatoire de Paris/CNRS/UPMC/Université Paris-Diderot, Meudon, France. \\ ${ }^{3}$ DLR, Institute of Planetary Research, Berlin, Germany. \\ ${ }^{4}$ IPAG - UGA, Grenboble, France.
}

Corresponding author email: mauro.ciarniello@inaf.it

Keywords: Comet 67P, Rosetta mission, water.

Rosetta was the first mission escorting a comet, 67P/Churyumov-Gerasimenko (67P hereafter), for two years along its orbit (August 2014 - September 2016). This allowed us to follow the evolution of the nucleus surface and activity as 67P was approaching the Sun from a heliocentric distance of 3.62 AU to $1.24 \mathrm{AU}$ (perihelion), and along the outbound orbit up to $3.8 \mathrm{AU}$. Systematic analysis carried out by means of the VIRTIS-M VIS channel $(0.24-1.04 \mu \mathrm{m},[1])$ across different areas on the nucleus shows that the observed spectral slope gets bluer with the comet approaching perihelion, while the original colors are progressively restored along the outbound orbit with the comet receding from the Sun. We interpret this color change with a variation of the water ice abundance on the surface. The described behavior suggests the presence of a seasonal cycle, with an increase of water ice on the nucleus with 67P approaching the Sun, followed by a progressive reduction after perihelion. Although this global trend is common to the whole surface, some variability in the temporal profile of the spectral slope for different areas is observed. We investigate whether these effects are related to the different insolation conditions and/or physical properties at different locations on the nucleus. These results will be also compared to the temporal variability of dust production with the aim to characterize the thermo-physical processes influencing the surface water ice content. In addition, we study the diurnal cycle of water ice [2] on the surface across the whole mission. We focus on the Imhotep region, investigating how this cycle is modulated by seasonal effects.

Acknowledgments:

We thank the following institutions and agencies for support of this work: Italian Space Agency (ASI, Italy), Centre National d'Études Spatiales (CNES, France), DLR (Germany), NASA (USA) Rosetta Program.

\section{References:}

[1] Coradini et al. (2007) - Space Sci. Rev., 128, 529.

[2] De Sanctis et al. (2015) - Nature, 525, 7570. 


\title{
Monte Carlo investigation of asteroid collisions statistics
}

\author{
Dell'Oro A.
}

INAF-Osservatorio Astrofisico di Arcetri.

Corresponding author email: aldo.delloro@inaf.it

Keywords: Monte Carlo technique, impact, kinematic conditions, asteroid.

The evaluation of the rate and the statistical distributions of the kinematic conditions of impacts among minor bodies is one of the fundamental prerequisites of any model of collisional evolution of such populations, or in general for any simulation of evolution of their physical (and dynamical) properties affected by the mutual collisions. Different strategies have been followed to address this problem. From one hand, semi-analytical approaches have been implemented on the basis of more or less sophisticated mathematical description of the orbital crossing. Such approaches have the advantage to be computationally fast, but their validity is limited to the simplest dynamical situations. From the other hand, impacts probabilities have been estimated extrapolating the number of close approaches recorded in N-body dynamical integrations. An alternative way consists in a Monte Carlo exploration of the space of phases of the system. This last approach has the merit to join the maximum flexibility for what concerns the dynamical hypotheses governing the motion of the bodies and the simplicity of implementation, but at the cost of longer computational times. In this presentation we show a new Monte Carlo technique, its applications and the current developments. 


\title{
Numerical simulations of thermal convection: applications to the dwarf planet Ceres
}

\author{
Formisano M. ${ }^{1}$, Federico C. ${ }^{1}$, De Sanctis M.C. ${ }^{1}$ \& Magni G. ${ }^{1}$ \\ ${ }^{1}$ INAF-IAPS.
}

Corresponding author email: michelangelo.formisano@inaf.it

Keywords: Ceres, internal structure, thermal properties.

Thermal convection is an important physical mechanism that affects the geophysical history of the bodies of the Solar System. In this work we focus on the role of the thermal convection in Ceres. Ceres is the biggest asteroid of the Main Belt, and it is characterized by a huge abundance of water ice in its interior as suggested, for example, by its low bulk density (about $2200 \mathrm{~kg} \mathrm{~m} \mathrm{-3,} \mathrm{[1]).} \mathrm{Its} \mathrm{surface} \mathrm{is} \mathrm{characterized} \mathrm{by} \mathrm{the} \mathrm{presence}$ of water ice patches, as revealed by the Visible and InfraRed (VIR) spectrometer [2, 3, 4]. Other several geological and geochemical evidence suggest the presence of water ice in Ceres interior: specific minerals on the surface produced by aqueous alteration [5,6,7,8], hydrogen detection [9], geomorphology [10].Recent theoretical models suggest a two-layers structure (e.g. [11, 12]): a rocky core (density $3400 \mathrm{~kg} \mathrm{~m}-3$ ) and a mantle $40 \mathrm{~km}$ thick with a density between 1200 and $1400 \mathrm{~kg} \mathrm{~m}-3$. The composition of the mantle, as well as the temperature gradient across the shell, affects the onset of the thermal convection. For this purpose, we developed a 2-D Finite-Element-Method (FEM) numerical code, which solves the Navier-Stokes equations coupled with the heat equation. Our numerical results provide physical constraints for the onset of the thermal convection. We also discuss about the possibility to produce domes by the thermal stress induced by the thermal convection.

\section{References:}

[1] Park et al. (2016) - Nature 537, p 515-517.

[2] Combe et al. (2016) - Science 353, aaf3010.

[3] Combe et al. (2018) - https://doi.org/10.1016/j.icarus.2017.12.008

[4] Raponi et al. (2018) - Science Advance 4.

[5] De Sanctis et al. (2016) - Nature, 536, 4.

[6] Ciarniello et al. (2017) - A\&A, 598, A130.

[7] Carrozzo et al. (2018) - Science Advance, 4.

[8] McSween et al. (2018) - MAPS, https://doi.org/10.1111/maps.13111

[9] Prettyman et al. (2017) - Science, 355, 55.

[10] Schmidt et al. (2017) - Nature Geoscience, 10, 338.

[11] Castillo-Rogez et al. (2018) - MAPS, 53, 1820.

[12] Fu et al. (2017) - Earth \& Planetary Science Letters, 476, 153. 


\title{
Carbonaceous near-Earth objects: a survey of the primitive material in the Solar System
}

\author{
Ieva S. ${ }^{1}$, Dotto E. ${ }^{1}$, Mazzotta Epifani E. ${ }^{1}$, Perna D. ${ }^{1,2}$, Micheli M. ${ }^{3}$, Brucato J.R. ${ }^{4}$, Poggiali G. ${ }^{4,5}$, \\ Antoniucci S. ${ }^{1}$, Valsecchi G.B. ${ }^{6,7}$ \& Perozzi E. ${ }^{8}$ \\ ${ }^{1}$ INAF - Osservatorio Astronomico di Roma, Rome, Italy. \\ ${ }^{2}$ LESIA - Observatoire de Paris, Meudon, France. \\ ${ }^{3}$ ESA NEO Coordination Centre, Frascati, Rome, Italy. \\ ${ }^{4}$ INAF - Osservatorio Astrofisico di Arcetri, Firenze, Italy. \\ ${ }^{5}$ Università degli Studi di Firenze, Sesto Fiorentino, Italy. \\ ${ }^{6}$ INAF - Istituto di Astrofisica e Planetologia Spaziale, Roma, Italy. \\ ${ }^{7}$ IFAC-CNR, Sesto Fiorentino, Italy. \\ ${ }^{8}$ ASI - Agenzia Spaziale Italiana, Rome, Italy.
}

Corresponding author email: simone.ieva@inaf.it

Keywords: Near Earth asteroids, spectroscopy, primitive carbonaceous material.

Primitive small bodies, such as carbonaceous Near-Earth Objects (NEOs), are thought to be coming from the water- and organic-rich outer regions of the proto-planetary disk. They likely contain primordial material from the proto-solar cloud, as well as elements issued from alteration processes. Their current physical and orbital properties provide information about the earliest processes that governed the formation and evolution of the proto-nebula at different solar distances [1], [2]. Moreover, recent exobiological scenarios for the origin of life on Earth invoke the exogenous delivery of organic matter to the early Earth due to an intense influx of organic-rich material after its formation [3], for which the most likely source is the impact of many NEOs formed in the outer Solar System [4]. We will present the results we have obtained during our latest observational campaigns to investigate the surface composition of several primitive bodies through photometric and spectroscopic observations in the visible and near-infrared range. We have found NEOs with dynamical and physical connection with outer Solar System bodies, making them particularly interesting for a detailed future investigation.

\section{References:}

[1] Morbidelli et al., (2008) - In The Solar System beyond Neptune, Eds. M.A. Barucci et al., Univ. Ariz. Press, 275-292.

[2] Vernazza et al. (2017) - AJ 153, 72, 1-10.

[3] de Marcellus et al. (2011) - ApJL, 727, L27, 1-6.

[4] Walsh K.J. et al. (2011) - Nature 475, 7355, 206-209. 


\title{
The surface variability of 3200 Phaethon: implication for its origin and evolution
}

\author{
Lazzarin M. ${ }^{1}$, Petropoulou V. ${ }^{2}$, Bertini I. ${ }^{1}$, La Forgia F. ${ }^{1} \&$ Migliorini A. ${ }^{3}$ \\ ${ }^{1}$ Department of Physics and Astronomy “G. Galilei”, University of Padova, Vicolo dell’Osservatorio 3, \\ I-35122, Padova, Italy. \\ ${ }^{2}$ Centro di Ateneo di Studi ed Attivit Spaziali "Giuseppe Colombo" (CISAS), University of Padova, Via Venezia 15, \\ I-35131, Padova, Italy. \\ ${ }^{3}$ INAF-IAPS, Via del Fosso del Cavaliere n. 100, 00133, Rome, Italy.
}

Corresponding author email: monica.lazzarin@unipd.it

Keywords: Phaethon, spectroscopy, surface compositional variability.

The Potentially Hazardous Asteroid 3200 Phaethon has been the subject of an intensive international observational campaign during its last close encounter with the Earth in December 2017. Phaethon is a very peculiar object which nature is not yet well understood: its asteroidal behaviour is accompanied by a dynamical connection to the Genimid meteor shower, typical cometary residuals. Classified as a B-type asteroid, different spectral slopes have been found by several authors in the wavelength region shortward $0.55 \mu \mathrm{m}$. The peculiarity of Phaethon has led JAXA to devote to its exploration the spatial mission Destiny+ to be launched in 2022. We participated to the international campaign by observing specroscopically in the visible region 3200 Phaethon in Dec. 2017 with the 1.22-m Asiago telescope obtaining interesting and unique results on its surface composition that is a significant variation in its spectral slope in the wavelength range [0.33-0.64] $\mu \mathrm{m}$. We found also for the first time a weak absorption band around $0.43 \mu \mathrm{m}$ and a comparison with meteorites has been performed. We discuss several scenarios that could be responsible for the variability obtained in particular the possible presence of a blue polar region. Last October 2018 we spectroscopically observed in the visible region also 2005 UD, a NEO dynamically connected with both Phaethon and the Geminids, suggesting a possible common origin of the three. The reduction of the data is ongoing and preliminary results will be presented. 


\section{P/Churyumov-Gerasimenko's dust activity from pre- to post- perihelion as detected by Rosetta/GIADA}

Longobardo A. ${ }^{1,2}$, Della Corte V. ${ }^{1,2}$, Rotundi A. ${ }^{1,2}$, Rinaldi G. ${ }^{1,2}$, Zakharov V.V. ${ }^{1,2}$, Ivanovski S.L. ${ }^{2,3}$, Formisano M. ${ }^{1}$, Palomba E. ${ }^{1,4}$, Dirri F. ${ }^{1} \&$ Palumbo P. ${ }^{1,2}$

${ }^{1}$ INAF-IAPS, via Fosso del Cavaliere 100, Rome, Italy.

${ }^{2}$ DIST-Università Parthenope, Centro Direzionale, Isola C4, 80143, Naples, Italy.

${ }^{3}$ INAF-OATS, via G.B. Tiepolo 11, 34143 Rome, Italy.

${ }^{4}$ ASI-SSDC, via del Politecnico snc, 00133 Rome, Italy.

Corresponding author email: andrea.longobardo@inaf.it

Keywords: Comet 67P, Rosetta mission, gas and dust activity.

We studied the dust activity of 67P/Churyumov-Gerasimenko, by analyzing the detections of the Rosetta/ GIADA instrument [1], in particular the fluffy and compact particles detected by the GDS and IS subsystems, respectively [2]. We retrieved the source regions of each dust particle detected, by applying the trace-back algorithm developed and validated for the first part of the Rosetta mission to 67P [3]. The algorithm assumes a radial motion, a uniform acceleration to a height of $11 \mathrm{~km}$ and a constant velocity above. The analysis was performed for different periods of the mission, that we label with 0 (1st inbound arc), 1 (2nd inbound arc), 2 (pre-perihelion), 3 (perihelion), 4 (1st post-perihelion stage) and 5 (2nd post-perihelion stage). As already found by [3], we confirm that the source of fluffy and compact particles is likely the same for periods 0,1 and 5. The lack of correlation between emission of fluffy and compact particles in the other periods is ascribed to the higher spacecraft altitude on the surface. This leads to a deficit of detected fluffy particles for two reasons: 1) loss of radial motion assumption, and consequent lower reliability of the obtained results; 2) lower speed of fluffy particles [3]. To check for a correlation between occurrence of fluffy agglomerates and surface morphology, as supposed by [4], we mapped the fraction of emitted fluffy particles in the different periods. Whereas at period 0 the fraction of fluffy particles is very similar in rough and smooth terrains, we observe a lack of fluffy particles in smooth regions at increasing cometary activity, as expected [4]. Otherwise, after perihelion fluffy particles are more abundant in smooth terrains. Simulations are in progress to verify if this behaviour can be explained by thermal inertia.

\section{References:}

[1] Colangeli et al. (2007) - Adv. Spa. Res. 3, 446-450.

[2] Della Corte et al. (2015) - A\&A 583, A10.

[3] Longobardo et al. (2018) - MNRAS, in press.

[4] Schulz et al. (2015) - Nature 518. 


\title{
Landslides on Comet 67P/Churyumov-Gerasimenko
}

\author{
Lucchetti A. ${ }^{1}$, Penasa L. ${ }^{2}$, Pajola M. ${ }^{1}$, Massironi M.. ${ }^{1,3}$, Brunetti M.T. ${ }^{4}$, Cremonese G. ${ }^{1}$ \& OSIRIS-REx Team \\ ${ }^{1}$ INAF-Astronomical Observatory of Padova, Italy. \\ ${ }^{2}$ CISAS- "G. Colombo", University of Padova, Italy. \\ ${ }^{3}$ Geosciences Department, University of Padova, Italy. \\ ${ }^{4}$ Research Institute for Geo-Hydrological Protection, Italian National Research Council, Perugia, Italy.
}

Corresponding author email: alice.lucchetti@inaf.it

Keywords: Comet 67P, Rosetta mission, surface propertie.

Mass movements are almost ubiquitous in the Solar System, with rockfall, avalanches or landslides that are observed not only on Earth, but also on multiple other bodies. The characteristics of the observed landslides (i.e. type of failure, geometry, velocity, etc.) are strongly influenced by several factors such as the initial mass position, the material and its mechanical properties, the topographic relief and the volatile content. In this work, we identified 26 landslides located on the surface of the comet 67P using OSIRIS Rosetta images. We investigate the landslide shapes and aspect ratios through the use of the high-resolution shape model of 67P. Assuming the height to runout length as an approximation for the friction coefficient of the cometary material, we find that the mapped mass failures behaves thoroughly different from the one observed on other Solar System icy bodies. Indeed, being the 67P high friction coefficients comparable, or even higher, than those found on Earth landslide, they imply a mechanically rocky-type behavior for the cometary material. This makes 67P, and comets in general, very peculiar objects that are mainly composed of ice and organics, but that are characterized by rocky-type properties rather than icy-type characteristics. 


\title{
An update on the YORP-Yarkowski evolution of asteroid families
}

\author{
Marzari F. ${ }^{1}$, Rossi A. ${ }^{2}$, Paolicchi P. ${ }^{3}$, Dall’Oro A. ${ }^{4}$, Scheeres D.J. ${ }^{5} \&$ Golubov O. ${ }^{6}$ \\ ${ }^{1}$ Università di Padova, Italia. \\ ${ }^{2}$ IFAC-CNR, Sesto Fiorentino (FI), Italia. \\ ${ }^{3}$ Università di Pisa, Italia. \\ ${ }^{4}$ Osservatorio Astrofisico di Arcetri, Firenze, Italia. \\ ${ }^{5}$ University of Colorado at Boulder, USA. \\ ${ }^{6}$ Kharkiv National University, Ukraine.
}

Corresponding author email: $\underline{\text { a.rossi@ifac.cnr.it }}$

Keywords: asteroid families, YORP-Yarkovsky effect, orbital and rotational evolution.

According to Golubov \& Scheeres (2018) the coupling between obliquity and spin rate evolution drives the rotation state of small bodies towards equilibria which can significantly influence the spin dynamics of asteroids. To have a full picture of this dynamics it is essential to consider the so-called Tangential YORP (TYORP) effect, caused by asymmetric light emission by boulders or other structures on the surface of an asteroid. Introducing these findings in a model which tracks the coupled orbital and rotational evolution of asteroid families, under the action of the Yarkowsky and YORP effects, we are able to recover the characteristic V-shape configuration in the semimajor axis vs absolute magnitude $(\mathrm{H})$ space. The time evolution of a number of synthetic families is explored with this model looking in detail at different size regimes and considering the influence of mutual collisions between the members of the family.

Looking at time snapshots of the V-shape evolution the YORP-eye hypothesis (Paolicchi and Knezevic, Icarus, 2016) is probed too.

References:

[1] Golubov \& Scheeres (2018) - The Systematic Structure of YORP Dynamics. In Lunar and Planetary Science Conference (Vol. 49)

[2] Paolicchi \& Knežević Z. (2016) - Footprints of the YORP effect in asteroid families. Icarus, 274, 314-326. 


\title{
Insights of the Vesta family and non-family members spectroscopic properties
}

\author{
Migliorini A. ${ }^{1}$, De Sanctis M.C. ${ }^{1}$, Lazzaro D. ${ }^{2}$, Lazzarin M. ${ }^{3}$, La Forgia F. ${ }^{3}$, Barbieri M. ${ }^{4} \&$ Mesa D. ${ }^{5}$ \\ ${ }^{1}$ IAPS-INAF, Rome. \\ ${ }^{2}$ Observatory of Rio. \\ ${ }^{3}$ University of Physics and Astronomy, Padova. \\ ${ }^{4}$ University of Atacama. \\ ${ }^{5}$ Observatory of Padova.
}

Corresponding author email: alessandra.migliorini@inaf.it

Keywords: V-type asteroids, spectroscopy, comparison with Vesta surface composition.

Recently the Dawn mission realized the most complete mapping of the surface composition of (4) Vesta, the unique large basaltic asteroid in the Main Belt, which has experienced an extensive geochemical differentiation $[1,2]$. However, in the last decade an increasingly large number of small asteroids with a possible basaltic composition, to be verified through spectroscopic investigation, has been identified. They can be grouped in diverse populations according to their location, from the NEAs region up to the outer belt [3]. During the last decade, we acquired spectra of more than 70 asteroids, selected among the putative V-type asteroids, and classified on the basis of their photometric colors in the visible [4, 5] or NIR [6]. We obtained mineralogical information for these asteroids in the wavelength range 0.3-2.5 $\mu \mathrm{m}$, observed during several runs at Telescopio Nazionale Galileo (La Palma) and Very Large Telescope (ESO, Paranal). Comparisons of the observed asteroids with Howardite-Eucrite-Diogenite meteorites and the Vesta's surface, as it resulted from the VIR/ Dawn spectrometer, allowed identifying possible links with regions on the Vesta surface. A better knowledge of the differences and similarities between V-type asteroids all around the Main Belt has, thus, been attained improving our understanding of the origin of basaltic material in the Solar System $[7,8,9,10]$.

In the present work, we summarize the results obtained in 10 years of observations of V-type asteroids, performed at Telescopio Nazionale Galileo and Very Large Telescope.

\section{References:}

[1] De Sanctis et al. (2012), Science.

[2] Ammannito et al. (2013), Meteoritics \& Planetary Science.

[3] Nesvorny et al. (2008), Icarus.

[4] Roig and Gil-Hutton et al., 2006, Icarus.

[5] Carvano et al. (2010), A\&A.

[6] Licandro et al. (2017), A\&A.

[7] De Sanctis et al. (2012a), MNRAS.

[8] De Sanctis et al. (2012b), A\&A.

[9] Migliorini et al. (2017), MNRAS.

[10] Migliorini et al. (2018), MNRAS. 


\title{
Scientific Analysis of the Size-Frequency Distribution of Boulders $\geq 10 \mathrm{~m}$ on Asteroid (101955) Bennu
}

\author{
Pajola M. ${ }^{1}$, Della Giustina D. ${ }^{2}$, Bennett C. ${ }^{2}$, Burke K. ${ }^{2}$, Lauretta D.S. ${ }^{2}$, Rizk B. ${ }^{2}$, Delbo M. ${ }^{3}$, Walsh K. ${ }^{4}$, \\ Brucato J.R. ${ }^{5}$, Dotto E. ${ }^{6}$, Bierhaus E.B. ${ }^{7}$, Campins H. ${ }^{8}$, Daly M. ${ }^{9}$, Elder C. ${ }^{10}$, Michel P. ${ }^{3}$, Molaro J. ${ }^{11}$, \\ Nolan M.C. ${ }^{2}$, Schwartz S.R. ${ }^{2,3}$ \& The OSIRIS-REx Team
}

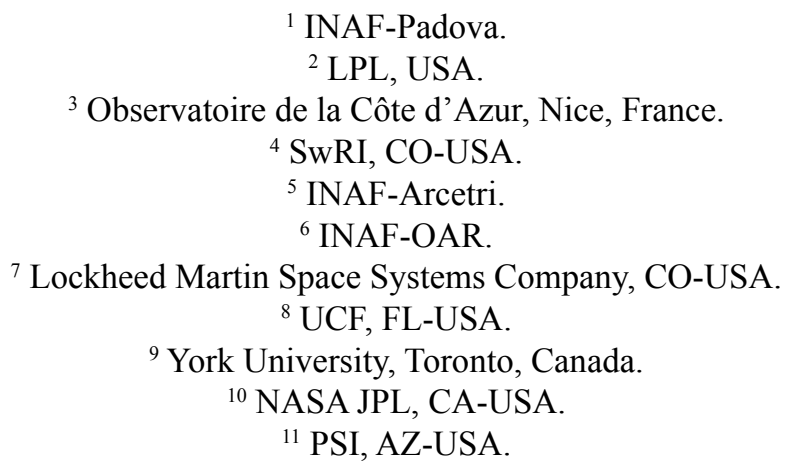

Corresponding author email: maurizio.pajola@inaf.it

Keywords: Asteroid Bennu, OSIRIS-REx mission, surface properties.

The Origins, Spectral Interpretation, Resource Identification, Security-Regolith Explorer (OSIRIS-REx) spacecraft reached its destination, the Apollo-type near-Earth asteroid (101955) Bennu [1], on December 3, 2018. During the Approach phase of the mission, the OCAMS PolyCam imager [2] acquired many images. The PolyCam dataset taken on November 13, 2018, has a spatial resolution of $2.0 \mathrm{~m}$. We used it to determine the first global size-frequency distribution (SFD) of boulders ${ }^{3} 10 \mathrm{~m}$ over the entire asteroid surface.

We will present our analysis of the SFD and compare the boulder spatial densities per square kilometer with those of other minor bodies $[3,4,5,6]$ to understand what processes created and/or degraded Bennu's boulders.

Acknowledgments:

M. Pajola was supported for this research by the Italian Space Agency (ASI) under the ASI-INAF agreement no. 201737-H.0. M. Delbo, P. Michel and S. R. Schwartz acknowledge support from the Centre National d'Études Spatiales, as well as the Academies of Excellence on Complex Systems and Space, Environment, Risk and Resilience of the Initiative d'EXcellence 'Joint, Excellent, and Dynamic Initiative' (IDEX JEDI) of the Université Côte d'Azur. This material is based upon work supported by NASA under Contract NNM10AA11C issued through the New Frontiers Program.

\section{References:}

[1] Lauretta et al. (2017) - OSIRIS-Rex: Sample Return from Asteroid (101955) Bennu. Space Sci. Rev. 212: 925-984.

[2] Rizk et al. (2018) - OCAMS: The OSIRIS-REx Camera Suite. Space Sci. Rev. 214:26.

[3] Dombard et al. (2010) - Boulders and ponds on the Asteroid 433 Eros. Icarus 210, 713.

[4] Küppers et al. (2012) - Boulders on Lutetia. PSS. 66, 71.

[5] Pajola et al. (2015) - SFD of boulders $\geq 7 \mathrm{~m}$ on comet 67P/Churyumov-Gerasimenko. A\&A. 583, A37.

[6] Pajola et al. (2016) - SFD distribution of boulders $\geq 10 \mathrm{~m}$ on comet 103P/Hartley 2. A\&A. 585, A85. 


\title{
The V-plot of asteroid families, and the relevance of the initial conditions: a preliminary analysis
}

\author{
Paolicchi P. ${ }^{1}$, Spoto F. ${ }^{2}$, Knezevic Z. ${ }^{3} \&$ Dell'Oro A. ${ }^{4}$ \\ ${ }^{1}$ Dipartimento di Fisica, Università di Pisa. \\ ${ }^{2}$ Université Cote d'Azur, OCA-CNRS, Lab. Lagrange (F). \\ ${ }^{3}$ Serbian Acedemy of Sciences and Arts, Belgrade (S). \\ ${ }^{4}$ INAF, Osservatorio di Arcetri, Firenze.
}

Corresponding author email: paolo.paolicchi@unipi.it

Keywords: Asteroid Families, Yarkovsky effect, Age determination.

In the so-called V-plot the proper semimajor axis of the members of an asteroid family is plotted vs. the inverse of its size (1/D). In the plot it is possible to obtain, through a fitting method, two lines, close to the left and right envelopes of the data, which allow also to perform age estimates. The lines may cross each other at some value of $1 / \mathrm{D}$ (obviously $1 / \mathrm{D}=0$ corresponds to a limit infinite size; formally we can extend the plot also to meaningless negative values).

If the $1 / \mathrm{D}$ value is positive we define the case as a "positive V-base" (the V-base is defined as the segment connecting the two lines at $1 / \mathrm{D}=0$ ); otherwise we can have a null or a negative $\mathrm{V}$-base. The crossing properties are connected to the size dependence of the Yarkovsky semimajor axis mobility, to the original ejection velocity field and to spin-original velocity correlations. We discuss the results of a preliminary analysis of the problem, which follows also the suggestions by Andrea Milani. 


\section{Aliphatic organics on comet 67P: from interstellar dust to pristine solar system material}

Raponi A. ${ }^{1}$, Ciarniello M., Filacchione G., Capaccioni F., De Sanctis M.C., Moroz L.V., Vinogradoff V., Tosi F., Arnold G., Quirico E., Mennella V., Beck P., Pilorget C., Pommerol A., Schröder S., Kappel D., Istiqomah I., Rousseau B. \& Poch O.

${ }^{1}$ IAPS-INAF, Rome, Italy.

Corresponding author email: andrea.raponi@inaf.it

Keywords: Comet 67P, Rosetta mission, astrochemistry.

The VIRTIS spectra of 67P/CG in the region between 2.9 and $3.5 \mu \mathrm{m}$ display a wide absorption band, which has been associated to the presence of organic compounds $[1,2]$. However, several instrumental effects have hindered, so far, the detailed interpretation of the molecules and compounds contributing to this band. In this work we revised the in-flight calibration of the VIRTIS-M-IR instrument onboard Rosetta spacecraft [3, 4] with the aim to improve: a) the detection of low-contrast spectral features and b) the radiometric accuracy. This updated calibration involves all 432 spectral channels $(\lambda)$ and 256 spatial samples. It includes: 1) the removal of artifacts associated to calibration residuals and/or incomplete flat field correction; 2 ) the reduction of the non-poissonian noise introduced by the readout electronics of the instrument; 3 ) a newer version of the radiometric calibration using stellar sources. Furthermore, we have modeled the thermal emission to remove the nucleus contribution at wavelengths in excess of $3.0 \mu \mathrm{m}$. This refined analysis shows a complex internal structure inside the wide 2.8-3.6 $\mu \mathrm{m}$ absorption feature. Clear evidence of the presence of aliphatic compounds is given by the $3.38,3.42$ and $3.47 \mu \mathrm{m}$ bands. This novel infrared detection of aliphatic species on a cometary surface allows a better understanding of formation and evolutionary processes by filling the observational gap between interstellar dust grains and solar system materials. The overall shape of the spectrum presents similarities with some outer solar system objects suggesting a genetic link.

\section{References:}

[1] Capaccioni et al. (2015) - Science, 347.

[2] Quirico et al. (2016) - Icarus, 272, 32-47.

[3] Coradini et al. (2007) - Space Sci. Rev., 128, 529.

[4] Filacchione (2006) - PhD thesis. 


\section{Analysis of a night side dust activity on comet 67P observed by VIRTIS-M: A new method to constrain the surface thermal inertia}

Rinaldi G. ${ }^{1}$, Formisano M. ${ }^{1}$, Kappel D. ${ }^{2}$, Capaccioni F. ${ }^{1}$, Bockelée-Morvan D. ${ }^{3}$, Cheng Y.-C. ${ }^{3}$, Vincent J-B. ${ }^{2}$, Deshapriya P. , Arnold G. ${ }^{2}$, Capria M.T. ${ }^{3}$, Ciarniello M. ${ }^{1}$, D'Aversa E. ${ }^{1}$, De Sanctis M.C. ${ }^{1}$, Doose L. ${ }^{4}$, Erard S. ${ }^{3}$, Federico C. ${ }^{1}$, Filacchione G. ${ }^{1}$, Fink U. ${ }^{4}$, Leyrat C. ${ }^{3}$, Longobardo A. ${ }^{1}$, Magni G. ${ }^{1}$ el al.

Corresponding author email: giovanna.rinaldi@inaf.it

Keywords: Comet 67P, thermal properties, Rosetta mission.

On 18 July 2015, near perihelion at a heliocentric distance of 1.28 au, the Visible InfraRed Thermal Imaging Spectrometer (VIRTISM) onboard the Rosetta spacecraft had the opportunity to observe dust activity in the inner coma with a view from the night (shadowed) side of comet 67P/Churyumov-Gerasimenko. At the time of the measurements presented in this work, we observe a dust plume that originates on the far side of the nucleus. We are able to identify the approximate location of its source at the boundary between the Hapi and Anuket regions, and we find that it has been in darkness for about 3 hours before the observation. Assuming this timespan equal to the conductive time scale, we obtain a thermal inertia in the range 27-36 TIU. These thermal inertias values can be used to check, with a 3-D Finite-Element-Method (FEM) numerical code, if the surface/subsurface temperatures are in agreement with the values found in literature. We explore three di erent configurations: 1) a layer of water ice mixed with dust beneath a dust mantle $5 \mathrm{~mm}$ thick with thermal inertia of $36 \mathrm{~J} \mathrm{~m}-2 \mathrm{~K}-1 \mathrm{~s}-0.5 ; 2$ ) the same structure but with thermal inertia of $100 \mathrm{~J} \mathrm{~m}-2 \mathrm{~K}-1 \mathrm{~s}-0.5 ; 3$ ) ice-dust mixture directly exposed. Among these three configurations, the first seems to be the most reasonable one, both for the low thermal inertia and for the agreement with the surface/subsurface temperatures found for the comet $67 \mathrm{P} /$ Churyumov-Gerasimenko. The spectral properties of the plume show that the visible dust colour ranged from $16+-4.8 \% / 100 \mathrm{~nm}$ to $13+-2.6 \% / 100 \mathrm{~nm}$, indicating that this plume has no detectable colour gradient. The plume morphology can be classified as a narrow jet with an estimated total ejected mass between 6 and 19 tons, assuming size distribution indices between -2.5 and -3 . 


\title{
Ceres' thermal inertia from Dawn data
}

\author{
Rognini E. ${ }^{1}$, Capria M.T. ${ }^{1}$, Tosi F. ${ }^{1}$, De Sanctis M.C. ${ }^{1}$, Ciarniello M. ${ }^{1}$, Longobardo A. ${ }^{1}$, Carrozzo F.G. ${ }^{1}$, \\ Raponi A. ${ }^{1}$, Frigeri A. ${ }^{1}$, Palomba E. ${ }^{1}$, Fonte S. ${ }^{1}$, Giardino M. ${ }^{2}$, Ammannito E. ${ }^{2}$, \\ Raymond C.A. ${ }^{3} \&$ Russell C.T. ${ }^{4}$ \\ ${ }^{1}$ INAF-IAPS, Via del Fosso del Cavaliere 100, 00133 Roma. \\ ${ }^{2}$ ASI, Via del Politecnico snc, 00133 Roma. \\ ${ }^{3}$ Institute of Geophysics and Planetary Physics, 3845 Slichter Hall, 603 Charles E. Young Drive, East, Los Angeles, \\ CA 90095-1567, USA. \\ ${ }^{4}$ NASA/Jet Propulsion Laboratory and California Institute of Technology, 4800 Oak Grove Drive, Pasadena,
} CA 91109, USA.

Corresponding author email: edoardo.rognini@inaf.it

Keywords: Ceres, Dawn mission, thermal properties.

Thermal inertia is a fundamental parameter that controls surface temperature variations of airless body, and is an indicator of history and type of the surface material. The Visible and InfraRed mapping spectrometer (VIR) [1] onboard the NASA DAWN mission has allowed to measure the surface temperatures of Vesta and Ceres (the instrument is sensitive to temperatures higher than $180 \mathrm{~K}$ [2]), and a thermal analysis has been done for Vesta, and then Ceres ([3],[4]). The thermal inertia is derived by comparing the observed temperatures with the theoretical temperatures calculated with a thermophysical model; Ceres' surface has been divided into sectors and for each of them the theoretical temperature curve (temperature as function of the local solar time) has been calculated, varying the parameters of conductivity and roughness (that is a measure of topography on the sub-pixel scale) until observed temperatures were reproduced. Our analysis indicates a low value of the average thermal inertia for areas with no special features, according to previous independent estimates [5]; in general, this could confirm the inversely proportional trend of the thermal inertia as a function of the diameter, observed for several asteroids of the main belt [6]. The analysis was extended to the faculae of the Occator crater and to the Haulani crater. The high thermal inertia in the Haulani crater is consistent with the higher compactness as seen by the framing camera, and the thermal anomaly in the central part of Cerealia facula could be explained by a higher thermal inertia and/or roughness.

References:

[1] De Sanctis et al. (2011) - Space Sci. Rev.

[2] Tosi et al. (2014) - Icarus.

[3] Capria et al. (2014) - Geophysical Research Letters.

[4] Rognini et al., Journal of Geophysical Research, in submission.

[5] Müller et al. (1998) - Astron. Astrophys.

[6] Delbo et al. (2008) - Planet. Space Sci. 


\title{
Analytical models for the dusty-gas near nucleus coma
}

\author{
Zakharov V.V. ${ }^{1,2}$, Ivanovski S.L. ${ }^{3}$, Della Corte V. ${ }^{1,2}$ \& Rotundi A. ${ }^{1,2}$
}

${ }^{1}$ INAF - Istituto di Astrofisica e Planetologia Spaziali, Ricerca Tor Vergata, Via Fosso del Cavaliere 100, 00133 Rome, Italy.

${ }^{2}$ Universitàdegli Studi di Napoli Parthenope, Dip. di Scienze e Tecnologie, CDN IC4, 80143 Naples, Italy.

${ }^{3}$ INAF - Osservatorio Astronomico di Trieste, Via Tiepolo 11, I-34143 Trieste, Italy.

Corresponding author email: zvv1661@yandex.ru

Keywords: Comets, gas and dust activity, theoretical models.

In the context of an increasing number of complex multiparametric dusty gas coma models it is convenient to construct a set of elementary models with a minimum number of parameters selected to represent the key processes acting on the dusty gas coma. The models outputs can be used as a reference evaluation of these processes with rough estimates of the resulting dusty gas properties. 
METEORS AND METEORITES, IDPS \& SAMPLES RETURNED FROM MINOR BODIES 


\title{
Graphite-based geothermometry of Almahata Sitta ureilites
}

\author{
Barbaro A. ${ }^{1}$, Domeneghetti M.C. ${ }^{1}$, Meneghetti M. ${ }^{2}$, Litti L. ${ }^{2}$, Fioretti A.M. ${ }^{3}$, Goodrich C. ${ }^{4}$, Shaddad M.H. \\ Alvaro M. ${ }^{1}$, \& Nestola F. ${ }^{6}$ \\ ${ }^{1}$ Dipartimento di Scienze della Terra e dell'Ambiente, UNIPV, Italy. \\ ${ }^{2}$ Dipartimento di Scienze Chimiche, UNIPD, Italy. \\ ${ }^{3}$ Ministero degli Affari Esteri e della Cooperazione Internazionale, Italy. \\ ${ }^{4}$ Lunar and Planetary Institute, USRA, TX USA. \\ ${ }^{5}$ Department of Physics and Astronomy, University of Khartoum, Sudan. \\ ${ }^{6}$ Dipartimento di Geoscienze, UNIPD, Italy.
}

Corresponding author email: anna.barbaro01@universitadipavia.it

Keywords: geothermometry; Almahata Sitta ureilite; Micro-Raman spectroscopy.

Almahata Sitta (AhS) is the first meteorite produced from impact of a known asteroid. The 2008TC3 asteroid was discovered on 6th October 2008 and tracked a few hours before the impact in Sudan [1]. AhS is defined as a "polymict breccias" and its fragments show a great lithological diversity of ureilitic rocks and enstatite, ordinary, carbonaceous and Rumuruti chondrites. We have investigated three ureilitic stones (AhS 209B, AhS 72 and AhS A135A) from the AhS meteorite by Micro-Raman Spectroscopy (MRS) to retrieve geothermometric information using the relatively recent approach adopted by [2]. This approach is based on the measurement of the Full Width at Half Maximum (FWHM) of the graphite G band that, by comparison to previous geothermometric approaches, can provide temperatures of high temperature environments (i.e._900$1000^{\circ} \mathrm{C}$ ). The Raman spectra of graphite from the samples were collected in order to retrieve the graphite crystallization temperature hereafter Tmax [2], and in turn to apply it to the ureilite parent body. In addition, the peak position of the graphite Raman spectra and the $I(D) / I(G)$ ratio ( $I=$ intensity; $D=D$ band; $G=G$ band) from MRS allowed us to interpret the investigated graphite as nanographite [3]. Our calculated temperatures are slightly higher than those reported by [2] on the ureilitic fragment $\# 7$ of AhS [e.g. $990( \pm 120)^{\circ} \mathrm{C}$ ]. However, if we account for the temperature uncertainties of this approach (i.e. $\pm 120^{\circ} \mathrm{C}$ ), our data do agree with those by [2] to better than one e.s.d. This range of temperatures agrees with the range of peak equilibration temperatures of ureilites recorded by pyroxene thermometry $[4 ; 5 ; 6]$.

References:

[1] Shaddad et al. (2010) - Meteorit Planet Sci.

[2] Ross et al. (2011) - Meteorit Planet Sci.

[3] Ferrari \& Roberson (2000) - Phis. Rev. B.

[4] Warren et al. (2012) - Meteorit and Planet Sci.

[5] Goodrich et al. (2013) - Meteorit Planet Sci.

[6] Herrin et al. (2010) - Meteorit Planet Sci. 


\title{
Assessment of astrometric performances for PRISMA all-sky cameras
}

\author{
Barghini D. ${ }^{1,2}$, Gardiol D. ${ }^{1} \&$ Carbognani A. ${ }^{1,3}$
}

${ }^{1}$ INAF - Osservatorio Astrofisico di Torino - Via Osservatorio 20, 10025 Pino Torinese, Italy.

${ }^{2}$ Università degli Studi di Torino - Dipartimento di Fisica - Via Pietro Giuria 1, 10125 Torino, Italy.

${ }^{3}$ Osservatorio Astronomico della Regione Autonoma della Val d'Aosta - Lignan, 3911020 NUS (AO), Italy.

Corresponding author email: dario.barghini@edu.unito.it

Keywords: PRISMA; all-sky camera; bright meteors.

The PRISMA all-sky cameras network is aimed at the detection of bright meteors and recovery of meteorites. The determination of the strewn field of possible fragments and orbital elements of the parent body are conditional on performances of the astrometric calibration of the instrument. We adapted the astrometric model proposed by Ceplecha, Borovička et al. [1,2] by modifying the given explicit parametrization and implemented an automatic reduction pipeline. Positioning precision and accurancy achieved are mainly affected by experimental conditions, typical of this kind of all-sky cameras, like undersampling of stars' PSF on reference frames and saturation of bright bolides. We discuss random and systematic deviations induced by this effects and compare performances of different positioning algorithm.

\section{References:}

[1] Ceplecha (1987) - Bulletin of the Astronomical Institutes of Czechoslovakia, 38, 222.

[2] Borovička, Spurny \& Keclikova (1995) - A\&AS, 112, 173. 


\title{
Classification of two meteorites from North-Western Africa (NWA), characterization of the trace elements found in phosphide inclusions and analysis of the crystalline nano-phases contained in the fusion crusts and/or the shock veins
}

\author{
Bellesi M. ${ }^{1}$, Giuli G. ${ }^{1} \&$ Santini C. ${ }^{1}$ \\ ${ }^{1}$ School of Science and Technology, Geology Division University of Camerino Via Gentile III da Varano, 62032 \\ Camerino.
}

Corresponding author email: manlio.bellesi@gmail.com

Keywords: NWA meteorites. phosphides, nanophases, fusion crusts.

Meteorites have long been recognized as a fundamental source of information in order to understand the formation process of minor and planetary bodies in the Solar System. Almost 40,000 samples are known and classified nowadays, with additional meteorites retrieved each year by scientific campaigns roaming across the hot and cold deserts of the Earth - Antarctica being the most targeted location. We have been focusing our attention to the most common class of meteorites, the ordinary chondrites, which account for about $95 \%$ of all falls. Here we show the classification of 2 speciments reportedly found near ZAGORA- according to a widely accepted scheme outlined in [1] and [2] - and preliminary data on the mineralogy of crystalline and nanocrystalline phases found within the fusion crust by means of powder X-ray Diffraction data and Field Emission Scanning Electron Microscope (FE-SEM) [3]. Moreover, we aim to determine the trace elements contained in phosphide inclusions, via LA-ICP MS . To our knowledge, a systematic study on the subject hasn't been carried out yet, so that we plan on expanding our analysis to several other ordinary chondrites.

References:

[1] Krot et al. (2003) - Classification of meteorites. In Meteorites, Comets and Planets (ed. A.M. Davis) Vol. 1, Treatise on Geochemistry (eds. H.D. Holland and K.K. Turekian), Elsevier, Oxford, pp. 83-128 (2003).

[2] Weisberg et al. (2006) - Systematics and Evaluation of Meteorite Classification, in Meteorites and the Early Solar System II, D. S. Lauretta and H. Y. McSween Jr. (eds.), University of Arizona Press, Tucson, pp.19-52.

[3] Chi M. (2018) - A closer look at shocked meteorites: Discovery of new high-pressure minerals, American Mineralogist, Vol. 103, pp. 1521-1522. 


\title{
Chemical and physical characteristics of a new chondrite from Draa Valley, Tata (Morocco)
}

\author{
Bonadiman C. ${ }^{1}$, Lugari C. ${ }^{2}$, Pelorosso B. ${ }^{1}$, Lanzafame G. ${ }^{3}$, Mancini L. ${ }^{3} \&$ Vaccaro C. ${ }^{1}$ \\ ${ }^{1}$ University of Ferrara, Department of Physics and Earth Sciences. \\ ${ }^{2}$ Saint-Gobain PPC Italia S.P.A. - Weber activity. \\ ${ }^{3}$ beamline SYRMEP Sincrotrone Trieste S.C.p.A. Basovizza, Trieste, Italy.
}

Corresponding author email: bdc@unife.it

Keywords: chondrite; H3-petological type; S-type asteroids.

A chondritic meteorite, named 6A, was discovered in 2010 in southern Morocco near the city of Tata. Microtextural features were characterized using optical and scanning electron microscopes whereas mineral chemical compositions were obtained with electron microprobe. Mineralogical information derived by thermoanalytical techniques combined with quantitative X-ray powder diffraction. Bulk major and trace elements were obtained by XRF and ICP-MS analyses.

This rock is formed by a silicate portion ( $80 \%$ in vol), composed by chondrules, individual grains of olivine, pyroxene, rare plagioclase and a very fine grain-matrix, and metallic portion ( $20 \%$ in vol) formed by $\mathrm{Fe}-\mathrm{Ni}$ alloy kamacite $(\mathrm{Ni}<7 \%$ )and $\alpha$-taenite (Ni: 29.36 - $30.98 \mathrm{wt} \%$.), Fe sulphides (S: $34.76-37.07 \mathrm{wt} \%$ ) and rare pentlantite (Ni: $2.61 \mathrm{wt} \%$.).

Olivine and pyroxene compositions (Fa: 17.35-18.57; mg\#entstatite: 0.83-0.84) suggest the group H, 3 as petrological type (1).

The chondrule/matrix ratio of approximately 30:80 with the majority of chondrules being type-I (average Fa =17). The DTG profile reveals the presence of the goethite which suggests moderate (W2) weathering grade (2).

Six chips of the meteorite were subsequently characterized by synchrotron radiation computed microtomography followed by a quantitative 3D image analysis to determine the petrographic features (shape, texture, and modal abundance) in the rock volume. We also used the helium ideal-gas pycnometry for acquiring bulk density of the same meteorite chips (3). Combining chemical data and measured physical parameters (bulk density and porosity) with 3D structure mapping we calculate the intrinsic density of the parent body. The obtained value: $\delta=4.18+0.04 \mathrm{~g} / \mathrm{cm}^{3}$ suggests a $\mathrm{S}$ type asteroid (3).

\section{References:}

[1] Scott \& Krot (2014) - Treatise on Geochemistry, Vol 1, pp. 65-137.

[2] Grossman \& Brearley (2005) - Meteori, and Planet. Sci. 40: 87-122.

[3] Consolmagno, Britt \& Macke (2008) - Chemie der Erde 68: 1-29. 


\title{
MuFiS - Multipurpose Fireball Software
}

\author{
Carbognani A.
}

INAF-Osservatorio Astrofisico di Torino/Osservatorio Astronomico della Valle d'Aosta.

Corresponding author email: albino.carbognani@inaf.it

Keywords: PRISMA; Bright fireball; MuFiS.

A software used by PRISMA network is presented. The most interesting feature of this program is its ability to determine semi-automatically the trajectory, orbit, dynamic models, dark flight and strewn field for every observed bright fireball. The entire process and the quality of the fit can be controlled by the user. This application was written in Matlab language using scientific modules. It is also possible to install the compiled version and work without Matlab. 


\title{
Effects of mineralogical and petrographic variability on the VIS-NIR spectral features of HED meteorites
}

\author{
Carli C. ${ }^{1}$, Pratesi G. ${ }^{2}$, Moggi-Cecchi V. ${ }^{2}$ \\ ${ }^{1}$ IAPS - INAF. \\ ${ }^{2}$ Dipartimento di Scienze della Terra - Università di Firenze. \\ ${ }^{3}$ Museo di Storia Naturale - Università di Firenze.
}

Corresponding author email: cristian.carli@inaf.it

Keywords: VNIR spectroscopy; HED; Vestoids.

Achondritic meteorites are associated to differentiated parental bodies. They represent an expression of several processes causing the formation and evolution, of crust, mantle and core. A family of achondrites was associated in the past to its parental bodies thanks to their spectral properties. These are Howardite-EucriteDiogenite (HED) which were identified as samples from 4Vesta (and its family) since 80s [1]. This relationship was made considering the visible to near infrared reflectance spectroscopy and it was strengthened by the results of the DAWN mission, e.g. [2].

HED are a large family, circa the $60 \%$ of achondrites, but only few of them was investigated by reflectance spectroscopy. Increasing the number of studied samples help to evaluate the effects of mineralogy and petrography from the reflectance spectra. This procedure is therefore able to increase our knowledge on both HED and their parental body.

We'll summarize the results of 35 samples measured at IAPS-INAF in the last years, in collaboration with the Museo di Storia Naturale dell'Università di Firenze and the Museo di Scienze Planetarie di Prato, correlating the spectral properties to compositions, petrography and, for selected samples, with the grain size. Spectra show reflectance between 0.05-0.5, and mafic minerals are clearly indicated by the presence of Fe2+ crystal field absorptions around 0.9 and $1.9 \mathrm{um}$. In general, both the pyroxene absorptions are present in the spectra with a variation in intensity. Spectra show also a variability between 1.1 and $1.5 \mathrm{um}$, whit an asymmetry present in eucrites or in olivine rich region. We'll discuss these results in comparison with previous [e.g. 3,4] and recent [e.g. 5] works and indications for spectral interpretation will be shown.

\section{References:}

[1] Feierberg \& Drake (1980) - Science 209.

[2] DeSanctis et al. (2012) - Science 336.

[3] Burbine et al. (2009) - MPS 48.

[4] Beck et al. (2011) - Icarus 216.

[5] Burbine et al. (2018) - JGR 123. 


\title{
VNIR spectroscopy of Olivine grains in Achondrites Meteorites
}

\author{
De Angelis S. ${ }^{1}$, Carli C. ${ }^{1}$, Ferrari M. ${ }^{1}$, Manzari P. ${ }^{1,2}$, Vinogradoff V. ${ }^{1,3}$, De Sanctis M.C. ${ }^{1}$, \\ Pratesi G. ${ }^{4} \&$ Moggi Cecchi V. ${ }^{5}$ \\ ${ }^{1}$ INAF-IAPS, V. Fosso del Cavaliere, 100, 00133, Roma - Italy. \\ ${ }^{2}$ ASI, V. del Politecnico, 00133, Roma - Italy. \\ ${ }^{3}$ Aix-Marseille University, PIIM UMR-CNRS 7345, Marseille, France. \\ ${ }^{4}$ Dip. Scienze della Terra, Università di Firenze, Via G. La Pira, 4, 50121 Firenze - Italy. \\ ${ }^{5}$ Museo di Storia Naturale, Università di Firenze, Via G. La Pira, 4, 50121 Firenze - Italy
}

Corresponding author email: simone.deangelis@inaf.it

Keywords: VNIR spectroscopy; olivine; achondrites.

Olivine is one of the most diffuse silicates in Solar System rocky bodies, being present in planets' mantles as well as clearly exposed on the surfaces of V-type and A-type asteroids in the Main Belt [1,2]. Olivine grains have also been found to be present in several comets and also in a variety of circumstellar disks [3], from the silicate bands in telescopic mid-infrared spectra. Furthermore, olivine in form of phenocrysts/xenoliths grains is a constant component in numerous types of meteorites, both chondrites and achondrites. A few previous studies focused on the IR investigation of olivine grains in primitive chondrite meteorites, while very few if any have conducted systematic IR investigations on olivine in achondrites [4,5]. Here, we present analyses performed by means of VNIR imaging spectroscopy on a suite of achondrites meteorites. We analyzed a Martian basaltic Shergottite (Dar Al Gani 489), an Ureilite (FRO 10063), an Olivine-Diogenite (North West Africa 6232) [6, RI3225] and a Pallasite (Esquel). Analyses were all performed on slab samples, in order to investigate coarse minerals grains/crystals and to preserve as much as possible the primary texture. The measurements were carried out by using the SPIM facility [7], imaging spectrometer spare of Dawn-VIR in use at INAF-IAPS, in the spectral range 0.4-5.1 $\mathrm{m}$ with spatial resolution of $38 \mathrm{um}$ on the sample. The analyses of olivine 1-um band shape and position from these samples show notable differences that can be addressed to variation in composition and in texture/crystal sizes.

\section{References:}

[1] Ammannito et al. (2013) - Nature, 504, 122-125.

[2] Reddy et al. (2005) - LPSC XXXVI, abs. 1375.

[3] Molster et al. (2002) - A\&A 382, 222-240.

[4] Morlok et al. (2006) - MAPS 41, N.5, 773-784

[5] Maras et al. (2001) - 64th Annual Met. Soc., abs. 5203.

[6] De Angelis S. et al. (2015) - Rev. Sci. Instrum., 86, 093101.

[7] Carli et al. (2018) - MAPS 53, 2228-2242. 


\title{
Non destructive pre-characterization of micrometeorites by $\mathrm{X}-\mu \mathrm{CT}$
}

\author{
Dionnet Z. ${ }^{1}$, Longobardo A. ${ }^{1,2}$, Rotundi A. ${ }^{1,2}$, Suttle M.D. ${ }^{3}$, Brunetto R. ${ }^{4}$, Folco L. ${ }^{3}$, Gemelli M. ${ }^{3}$, \\ Della Corte V. ${ }^{1,2} \&$ King A. ${ }^{5}$ \\ ${ }^{1}$ DIST-Università Parthenope, Centro Direzionale Isola C4, 80143 Naples, Italy. \\ ${ }^{2}$ INAF-IAPS, via Fosso del Cavaliere 100, 00133 Rome, Italy. \\ ${ }^{3}$ DST-Università di Pisa, via S. Maria 53, 56126 Pisa. \\ ${ }^{4}$ IAS-Universitè Paris Sud, Batiment 121, Paris, France. \\ ${ }^{5}$ Psiche beamline, Synchrotron SOLEIL, Orne des Meurisiers, France.
}

Corresponding author email: dionnet.zelia@wanadoo.fr

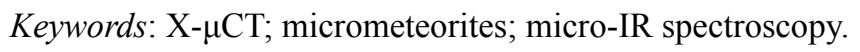

Micrometeorites dominate the influx of extraterrestrial material to Earth and provide samples of a variety of dust-producing bodies in the Solar System. These make them very valuable for analysis in the laboratory and can help us to provide constraints for modeling the source regions and dynamic evolution of the cosmic-dust complex in the primitive Solar System [1].

The framework of this project is to study mirometeorites through a sequence of analytical techniques [2]: X tomography, micro-IR spectroscopy and FESEM-EDX. X-ray $\mu \mathrm{CT}$ is a valuable technique to do a pre-characterization of both inside and outside of these precious samples. Non-invasive $\mu C T$ will provide initial information on phase composition, microporosity [3], microfabric, fracturing and other microstructures, identification of portions of the sample that may require special attention in the subsequent analyses/device analytical strategies. Some preliminary study has already been led on one sample and reveal structures and heterogeneity.

References:

[1] Folco \& Cordier (2015) - European Mineralogical Union Notes in Planetary Mineralogy, 15: 253-297.

[2] Longobardo et al. (2019) - this congress.

[3] Consolmagno et al. (2008) - Chemie der erde 68, 1-29.

Support: MIUR (PNRA16_00029 and PRIN 2015 - 20158W4JZ7) 


\title{
PRISMA italian fireball network: status update
}

\author{
Gardiol D. \& the PRISMA collaboration
}

INAF-Osservatorio Astrofisico di Torino \& the PRISMA collaboration.

Corresponding author email: daniele.gardiol@inaf.it

Keywords: PRISMA; fireball network; FRIPON.

Since 2017 we are deploying a network of all-sky cameras in Italy to monitor fireballs and bolides. Currently more than 30 cameras are operational and a dozen are in installation phase. The PRISMA (Prima Rete Italiana per la Sorveglianza sistematica di Meteore e Atmosfera) network is connected to the french project FRIPON. We present the status of the network and the most recent developments made on data analysis as well as in the field of outreach and educational activities. 


\title{
New compositions of shock-synthesized AICuFe icosahedral quasicrystals: implications for the impact origin of natural quasicrystals in the Khatyrka meteorite
}

\author{
Hu J. ${ }^{1}$, Asimow P.D. ${ }^{1}$, Ma C. ${ }^{1}$ \& Bindi L. ${ }^{2}$ \\ ${ }^{1}$ Division of Geological and Planetary Sciences, California Institute of Technology, USA. \\ ${ }^{2}$ Dipartimento di Scienze della Terra, Università degli Studi di Firenze, Italy.
}

Corresponding author email: luca.bindi@unifi.it

Keywords: Quasicrystal; Khatyrka meteorite; AlCuFe icosahedral.

Quasicrystals (QCs) produced by shock recovery experiments shine light on the impact origin of natural QCs in the Khatyrka meteorite [1,2]. The compositions of these synthetic AlCuFe QCs have so far been similar but not identical to natural icosahedrite $\left(\mathrm{Al}_{63} \mathrm{Cu}_{24} \mathrm{Fe}_{13}\right)$ and the unnamed i-phase II $\left(\mathrm{Al}_{62} \mathrm{Cu}_{31} \mathrm{Fe}_{7}\right)$ [3]. Here we present the results of two new shock recovery experiments (wave reverberation reached peak shock pressure $\sim 24 \mathrm{GPa}$ in both shots) on $\mathrm{Al}_{63} \mathrm{Cu}_{24} \mathrm{Fe}_{13}$ icosahedrite powder and a bonded granular mixture with a gradient in composition from $\mathrm{Al}$ and $\mathrm{Cu}$. Two new icosahedral QCs compositions were found and their structures studied by EBSD. About half of the shocked i- $\mathrm{Al}_{63} \mathrm{Cu}_{24} \mathrm{Fe}_{13}$ in $\mathrm{S} 1250$ decomposed into a new icosahedral QC with composition of $\mathrm{Al}_{65} \mathrm{Cu}_{19} \mathrm{Fe}_{16}$ plus an $\mathrm{Al}_{52} \mathrm{Cu}_{44} \mathrm{Fe}_{4}$ bcc alloy. Experiment $\mathrm{S} 1253$ sampled the full compositional range of the Al-Cu join in contact with 304 stainless steel. Surprisingly, the Al-rich region did not produce QCs. However, the intermediate $\mathrm{Al}-\mathrm{Cu}$ mixture reacted with the steel chamber to generate $\mathrm{i}-\mathrm{Al}_{62} \mathrm{Cu}_{30} \mathrm{Fe}_{7} \mathrm{Cr}_{1}$, co-existing with $\mathrm{Al}_{2} \mathrm{Cu}$ (khatyrkite) and $\mathrm{Al}_{3} \mathrm{Cu}_{2}$ (probably stolperite) alloys. The shock induced decomposition of icosahedrite in S1250 is similar to those observed in static high-pressure experiments from 3-21 GPa [4]. However, in our shock experiment Fe preferentially partitions into the quasicrystal and $\mathrm{Cu}$ into the alloy, contrary to the behavior in the static experiment. Conceivably this results from the effects of shear flow in the shock synthesis, stabilizing new compositions of icosahedral QC. More importantly, the synthesized i-phase II in S1253 is a near-exact compositional, textural, and assemblage match to the natural i-phase II occurrence in the Khatyrka meteorite.

\section{References:}

[1] Asimow et al. (2016) - PNAS, 113, 7077.

[2] Oppenheim et al. (2017) - Sci. Rep., 7, 15629.

[3] Bindi et al. (2016) - Sci. Rep., 6, 38117.

[4] Stagno et al. (2017) - PEPI, 271, 47. 


\title{
Infrared spectroscopy of micrometeorites' interior and exterior
}

\author{
Longobardo A. ${ }^{1,2}$, Dionnet Z. ${ }^{1,2}$, Rotundi A. ${ }^{1,2}$, Suttle M.D. ${ }^{3}$, Brunetto R. ${ }^{4}$, Folco L. ${ }^{3}$, \\ Gemelli M. ${ }^{3} \&$ Della Corte V., \\ ${ }^{1}$ INAF-IAPS, via Fosso del Cavaliere 100, 00133 Rome, Italy. \\ 2 DIST-Università Parthenope, Centro Direzionale Isola C4, 80143 Naples, Italy. \\ ${ }^{3}$ DST-Università di Pisa, via S. Maria 53, 56126 Pisa. \\ ${ }^{4}$ IAS-Universitè Paris Sud, Batiment 121, Paris, France.
}

Corresponding author email: andrea.longobardo@inaf.it

Keywords: Infrared spectroscopy; micrometeorites; meteorite interior and exterior.

The analysis of micrometeorites at sub-mm scale is fundamental to characterize extraterrestrial materials. In this and other works [1], we are proposing an approach based on a multi-technique analysis (i.e. spectroscopy, Raman spectroscopy, SEM, X-ray tomography) to carry on this kind of study.

Here we focus on infrared spectroscopy (2.5-12.5 um) performed on an unmelted micro-chondrite. In order to highlight compositional difference between the meteorite's exterior (which is affected from interaction with terrestrial atmosphere) and interior (which instead reflects the original composition), we acquired a first hyperspectral image, then compressed the chondrite in a diamond compression cell, and then acquired a new hyperspectral image of the pressed micrometeorite (in order to study the particle's inside).

A preliminary analysis revealed that the IR reflectance of the interior is on average three times larger than the exterior, due to the fusion crust, created during the atmosphere passage, which darkened the external layer. The main constituent of the interior is olivine, as revealed by the triple reflectance peak centered at $11.5 \mathrm{um}$, i.e. the olivine reststrahlen bands. Other two reflectance peaks are observed at about 9.2 and 9.9 um: since these peaks are observed also on the meteorite's exterior spectra, they should be due to weathering products. Jarosite is a good candidate, also due to the absorption observed at about 3 microns.

A comparison between the interior and exterior's spatial distributions of positions/heights/depth of most prominent spectral features is in progress.

Support: MIUR (PNRA16_00029 and ID 20158W4JZ7 - PRIN 2015)

References:

[1] Dionnet et al. (2019), this congress. 


\title{
New data and revision of classfication of the Sinnai meteorite (Cagliari)
}

\author{
Lugari C. ${ }^{1}$, Bonadiman C. ${ }^{2}$, Cruciani G. ${ }^{3}$, Franceschelli M. ${ }^{3}$, Marchi M. ${ }^{3} \&$ Tassinari R. ${ }^{2}$ \\ ${ }^{1}$ Saint-Gobain PPC Italia S.P.A. - Weber activity. \\ ${ }^{2}$ University of Ferrara, Department of Phisics and Earth Sciencs. \\ ${ }^{3}$ University of Cagliari, Department of Chemical and Geological Sciences.
}

\section{Corresponding author email: christian.lugari@alice.it}

Keywords: SINNAI meteorite; ordinary chondrite; H6-petrological type.

The Sinnai meteorite which fell on February 19th 1956 near Sinnai village in Sardinia. On the basis of Fa content in olivine (18 mol\%) the meteorite is classified as H6-petrological type.On the basis of the new chemical data of bulk, mineral major and trace element analyses, textural and physical characteristic.New thin section and mirrored chip, were analyzed for mineral chemistry and using scanning electron microscopes and electron microprobe.The bulk major and trace element abundances were obtained using XRF and ICP-MS. Petrographic analyses indicate a moderate shock stage (S3) condition, confirmed by clinoenstatite lamellae and opaque shock veins with feeble evidence of oxidation. The rock consists of chondrules (64 vol\%) up to $1.0 \mathrm{~mm}$ in size, large olivine and low Ca-pyroxene crystals, a fine-grained matrix (20 vol\%) and a metallic portion (16 vol\%).The matrix is made up of single olivine, pyroxene, rare plagioclase, rare merrillite and apatite. Fe-Ni alloy (kamacite-taenite phase) and Fe-sulfurs (taenite) are the component of the metallic portion. The Fa content in chondrules and singles olivine from the matrix are $15.7 \mathrm{~mol} \%$ and $12.1 \mathrm{~mol} \%$ respectively. Low-Ca pyroxenes (Wo 0.4-2.5 wt\%) is the dominant pyroxene in the meteorite. Glassy patches in chondrules occur associated with plagioclase (An 11-22 wt\%) and rarer tiny phosphates (Na2O 2.74-3.20 wt\%). Fe-Ni alloy is formed by kamacite (Ni 4.3-6.7 wt\%) and taenite (Ni 31.0-53.1 wt\%). Glasses are rich in $\mathrm{CaO}$ (1.65$4.00 \mathrm{wt} \%)$, and rather poor in $\mathrm{FeO}(0.62-5.50 \mathrm{wt} \%)$ and $\mathrm{MgO}(0.75-16.4 \mathrm{wt} \%)$. On the basis of $\mathrm{Mg} / \mathrm{Si}(0.906)$ and $\mathrm{Al} / \mathrm{Si}(0.083)$ ratios, the Sinnai meteorite is an ordinary chondrite, undistinguishable between $\mathrm{H}, \mathrm{L}$ and LL.Comparing with the average H-type, this meteorite shows moderate REE enrichment.The new data from our analysis suggest a new classification for this meteorites as H group, 3 petrographic type 1.

\section{References:}

[1] Brearley \& Jones (1988) - In “Plan. Mat.” J.J. Papike ed., Min. Soc. of Am. 


\title{
44Ti activity in meteorites and assessment of the long-term heliomagnetic field variation
}

\author{
Mancuso S. ${ }^{1}$, Taricco C. ${ }^{1,2}$, Colombetti P. ${ }^{2}$, Rubinetti S. ${ }^{1,2}$, Sinha N. ${ }^{3}$, Bhandari N. ${ }^{4}$, \\ Barghini D. ${ }^{1,2} \&$ Gardiol D. ${ }^{1}$ \\ ${ }^{1}$ Istituto Nazionale di Astrofisica, Osservatorio Astrofisico di Torino, Strada Osservatorio 20, Pino Torinese 10025, \\ Italy. \\ ${ }^{2}$ Dipartimento di Fisica, Universit’a di Torino, Via P. Giuria 1, 10125 Torino, Italy. \\ ${ }^{3}$ Wentworth Institute of Technology, Boston, MA, USA. \\ ${ }^{4}$ Science and Spirituality Research Institute, Navrangpura, Ahmedabad, India.
}

Corresponding author email: $\underline{\text { salvatore.mancuso@,inaf.it }}$

Keywords: 44Ti, meteorites, heliospheric magnetic field (HMF), 10Be.

The very low level of 44Ti activity in several meteorites fallen in the last 250 years was determined by using gamma-ray spectrometers (HPGe $+\mathrm{NaI}$ ) located in the underground laboratory of Monte dei Cappuccini (INAF-OATo) in Torino, Italy. This dataset provides a powerful independent tool to reconstruct the temporal dependence of the long-term evolution of the heliospheric magnetic field (HMF) in the past. When compared with sunspot-based, geomagnetic-based and 10Be-based composite reconstructions, the independent HMF variation obtained in this work is in agreement with the 10Be-based reconstruction while the sunspot-based and geomagnetic-based reconstructions appear to underestimate the slope of the overall trend, resulting in a flatter trend than evinced from our data. New measurements of the 44Ti activity in meteorites that have been recently acquired by our group are ongoing through a much improved gamma-ray spectrometer. These data will extend the investigated period by more than one decade. 


\title{
Bubble size distribution of microtektites from the Australasian strewn field
}

\author{
Marinucci W. ${ }^{1}$, Arzilli F. ${ }^{2}$, Mancini L. ${ }^{3}$, Cicconi M.R. ${ }^{4}$, Folco L. ${ }^{5}$, Glass B.P. ${ }^{6}$, Koeberl C. ${ }^{7}$, \\ Pratesi G. ${ }^{8}$ \& Giuli G. ${ }^{1}$
}

${ }^{1}$ School of Science and Technology-Geology division, University of Camerino, 62032 Camerino, Italy.

${ }^{2}$ School of Earth and Environmental Sciences, University of Manchester, Manchester, UK.

${ }^{3}$ Elettra-Sincrotrone Trieste S.C.p.A., Basovizza, Trieste, Italy.

${ }^{4}$ Department Werkstoffwissenschaften, Lehrstuhl für Glas und Keramik, Universität Erlangen-Nürnberg, Erlangen, Germany.

${ }^{5}$ Dipartimento di Scienze della Terra, Università di Pisa, Pisa, Italy.

${ }^{6}$ Department of Geological Sciences, University of Delaware, Newark, DE 19716, USA.

${ }^{7}$ Department of Lithospheric Research, University of Vienna, Vienna, Austria; and Natural History Museum, Vienna, Austria.

${ }^{8}$ Dip. Scienze della Terra, Università di Firenze, Firenze, Italy.

Corresponding author email: gabriele.giuli@unicam.it

Keywords: bubble size, microtektites, nucleation, disequilibrium.

In this study we determined bubble size distribution of a set of microtektites from the Australasian strewn field recovered from both deep ocean drilling and from continental environment in Antarctica (TAM). The number of bubbles is, with few exceptions, generally small, and all of them display a very high sphericity. 12 Australasian microtektites display a variable number of bubbles from 0 to 199, with only 3 samples displaying a relatively high number of bubbles (48, 109 and 199). 4 TAM display no bubbles, and one TAM displays 3 bubbles. Data for Australasian microtektites are compared with those of 3 North American microtektites, 3 Ivory Coast microtektites, and few tektite fragments.

The bubble size distribution (BSD) of tektites and microtektites can be described by a power law which is indicative of a bubble nucleation and growth in disequilibrium conditions. BSD data indicates that the evolution of the bubbles within the tektites and microtektites analysed was affected by coalescence. 


\title{
The Sannio meteorite: a new chondrite from Southern Italy
}

\author{
Moggi Cecchi V. ${ }^{1}$, Pratesi G. ${ }^{2}$, Ghiara M.R. ${ }^{3}$, Rossi M. ${ }^{3}$, Gardiol D. ${ }^{4} \&$ Ascione G. ${ }^{5}$ \\ ${ }^{1}$ Museo di Storia Naturale-SMA, Università degli Studi di Firenze, Via La Pira, 4, 50121 Firenze. \\ ${ }^{2}$ Dipartimento di Scienze della Terra, Università degli Studi di Firenze,Via La Pira,4, 50121 Firenze. \\ ${ }^{3}$ Centro Musei Scienze Naturali, Università Federico II, Via Mezzocannone, 8, 80134 Napoli. \\ ${ }^{4}$ Istituto Nazionale di Astrofisica, Osservatorio Astrofisico di Torino, via Osservatorio 20, 10025 Pino Torinese. \\ ${ }^{5}$ Associazione Sky Sentinel, Via Leone, 28, San Nicola la Strada, 81020 Caserta.
}

Corresponding author email: vanni.moggicecchi@unifi.it

Keywords: Sannio, italian meteorite, chondrite, Real Museo Mineralogico.

Italian meteorites are extremely rare since only 41 meteorites, both finds and falls, have been officially approved by the Meteoritical Society. Among these 25 are ordinary chondrites. We hereby report the discovery of a new H4 chondrite that fell in the Campanian area of Sannio, near Caserta in 1810. The specimen consists of two small pieces totally weighing 15 grams and displaying a partial fusion crust covering a gray, microgranular texture. It belongs to the collections of the Real Museo Mineralogico of the University of Naples Federico II (catalogue $\mathrm{N}^{\circ} 16433$ - E 4972, Monticelli collection) and is described in the old catalogues as Aerolite, Sannio, 1810. Since its name doesn't appear neither in the Meteoritical Bulletin database nor in the Catalogue of Meteorites [1], it can be considered a new meteorite. In order to confirm its extraterrestrial nature a chip of the specimen (cat. RI3346) was sent to the Museo di Storia Naturale of the University of Florence. The optical microscopy analysis performed on a thin section evidenced a chondritic texture with chondrules of various types (poikilitic PO, POP, BO, RP, C, GO) and ranging in size from 300 to 450 microns set in a fine-grained matrix made of olivine and orthopyroxene. Electron microscopy (SEM) allowed to determine an unequilibrated composition of olivine and orthopyroxene as well as the presence of three different $\mathrm{Fe}, \mathrm{Ni}$ alloys, chromite and apatite. EMPA minerochemical analyses on major and minor elements confirmed the unequilibrated composition of olivine which ranges from Fa0.6 in PO chondrules to Fa19.6 in the matrix. A PP chondrule with low-Ca pyroxene (En3.2Fs96.4Wo0.4) rimmed by diopside (En3.9Fs61.5Wo34.5) has also been observed. Weathering grade and shock stage are low (W0, S2). Based on textural and minerochemical data the meteorite can be tentatively assigned to the $\mathrm{H} 4$ group of chondrites.

\section{References:}

[1] Grady (2000) - Catalogue of Meteorites, CUP, 696 pp.

[2] Grady et al. (2013) - Atlas of Meteorites, CUP, 384 pp. 


\title{
MM 5.29: a unique CV-like micrometeorite as evidence of hydrothermal activity on CC parent bodies
}

\author{
Nava J. ${ }^{1}$, Suttle M.D. ${ }^{2}$, Carli C. ${ }^{3}$, Spiess R. ${ }^{1}$, Folco L. ${ }^{2}$, Najorka J. ${ }^{4}$, Palomba E. ${ }^{3} \&$ Massironi M. ${ }^{1}$ \\ ${ }^{1}$ Department of Geosciences, University of Padova, Padova, Italy. \\ ${ }^{2}$ Dipartimento di Scienze della Terra, Università di Pisa, 56126 Pisa, Italy. \\ ${ }^{3}$ IAPS-INAF, Istituto Nazionale di Astrofisica e Planetologia Spaziali, Roma, Italy. \\ ${ }^{4}$ Core research laboratories, Department of Earth Science, The Natural History Museum, Cromwell Rd, London SW7 \\ 5BD, UK.
}

Corresponding author email: jacopo.nava@phd.unipd.it

Keywords: CV-like micrometeorite; hydrotemal processes; thermal metamorphism.

The micrometeorite MM5.29 is a unique sample recovered from Frontier Mountain $\left(72^{\circ} 59^{\prime} \mathrm{S}-160^{\circ} 20^{\prime} \mathrm{E}\right)$ by the Programma Nazionale delle Ricerche in Antartide (PNRA) [1] because it shows clear features related to the $\mathrm{CV}$ chondrites and to extensive thermal metamorphism in presence of fluids. The sample has platy orientated Fe-rich olivine matrix in which andradite crystals surrounded by diopside and jarosite are widely distributed. Only two other samples have been recognized as CV-like micrometeorite ([2], [3]) among many others with a carbonaceous-like composition (e.g [4]). With this study we are expanding the knowledge in the micrometeorites compositional range with the first unambiguous $\mathrm{CV}$-like micrometeorite that also allows us to go deeper into the thermal metamorphism and hydrothermal events that took place on the CV parent body-(ies). Furthermore, given that micrometeorites come from the asteroid belt [5] and cometary sources [6-7-8], the study of this sample can be a very useful and an important support to the space mission with C type asteroids and comets as a target (e.g. NASA-Dawn, Hayabusa 2, Osiris-Rex, Rosetta) giving possible insights into their composition and geological evolution. In particular the MM5.29 shows NIR spectra close to the average NIR spectra of Ceres [9] with the diagnostic bands at $2.7 \mu \mathrm{m}, 3.1 \mu \mathrm{m}$ and $\sim 3.4 \mu \mathrm{m}$ [10]. This micrometeorite may thus give important and exclusive information on the hydrothermal activity that the dwarf planet Ceres suffered.

\section{References:}

[1] Folco et al. (2008) - Geology.

[2] Genge et al. (2010) - MetSoc.

[3] Van Ginneken et al. (2012) - Met. \& Planet. Sci.

[4] Cordier et al. (2018) - Geochim. Cosmochim. Acta.

[5] Carrillo-Sànchez et al. (2015) - Geophys. Res. Lett.

[6] Dobrică et al. (2012) - Geochim. Cosmochim. Acta.

[7] Imae (2012) - Proc. Internat. Astron. Union.

[8] Noguchi et al. (2015) - Earth \& Planet. Sci. Lett.

[9] Nava et al. (2018) - ESPC.

[10] De Sanctis et al. (2015) - Nature. 


\title{
Fusion crust and atmospheric entry of ordinary chondrites, a comparison between experiments and nature
}

\author{
Pittarello L. ${ }^{1}$, Goderis S. ${ }^{2}$, Soens B. ${ }^{2}$, McKibbin S.J. ${ }^{3}$, Bariselli F. ${ }^{4}$, Barros Dias B.R. ${ }^{4}$, Helber B. ${ }^{4}$, Giuli G. ${ }^{5}$, \\ Lepore G.O. ${ }^{6}$, Yamaguchi A. ${ }^{7}$, Roszjar J. ${ }^{8}$, Debaille V. ${ }^{9}$, Koeberl C. ${ }^{8}$, Magin T. ${ }^{4}$ \& Claeys P. ${ }^{2}$ \\ ${ }^{1}$ University of Vienna, Austria. \\ ${ }^{2}$ VUB, Belgium. \\ ${ }^{3}$ University of Potsdam, Germany. \\ ${ }^{4}$ von Karman Institute (VKI), Belgium. \\ ${ }^{5}$ University of Camerino, Italy. \\ ${ }^{6}$ ESRF, France. \\ ${ }^{7}$ NIPR, Japan. \\ ${ }^{8} \mathrm{NHM}$, Austria. \\ ${ }^{9} \mathrm{ULB}$, Belgium.
}

Corresponding author email: lidia.pittarello@univie.ac.at

Keywords: ordinary chondrites; fusion crust; melt.

Meteorite fusion crusts are quenched melt layers formed during meteoroid atmospheric entry, mostly preserved as coatings on the meteorite surface. Atmospheric entry is a complex process, involving extreme heating and interaction between the mostly chemically reduced asteroidal material and atmospheric gases, including oxygen. Constraining this process would contribute to better understanding of features observed in cosmic spherules and meteorites, and to calibrating experiments and numerical models on atmospheric (re) entry of various materials, including extraterrestrial matter and space debris.

This work presents the results of petrographic and geochemical investigations on both natural and experimentally produced fusion crusts in H-type ordinary chondrites. As natural samples, the fusion crusts of the Antarctic meteorites Asuka (A) 09004 and A 09502, both classified as H5, were selected for their extensive crystallization of olivine, which recorded the local environmental conditions through the Earth's atmosphere. For the experiments, a high enthalpy facility has been used, applying several setups, and melting both a terrestrial basalt, as meteoroid analogue, and a fragment of the ordinary chondrite El Hammami (H5). Comparison of the chemical and petrographic features between the natural and the experimental glass confirmed that the selected experimental setup successfully reproduced the conditions likely encountered by asteroidal material during atmospheric entry, with melting, vaporization of highly volatile elements, and possible local condensation effects. The crystallization of skeletal magnetite, the evolution of olivine composition in the overgrown rim, and models on the oxygen fugacity based on the Fe3+/Fetot ratio measured by XANES (X-ray absorption near edge structure) technique, all point to a redox equilibrium, mostly controlled by the temperature, reached in seconds during melting, and yielding oxidation of the relatively reduced chondrite. 
EXPERIMENTAL AND LABORATORIAL PLANETOLOGY 


\title{
EURO-CARES: Conceptual design and procedures for a European sample curation facility for sample return
}

\author{
Brucato J.R., Meneghin A. \& the EURO-CARES Team \\ INAF - Osservatorio Astrofisico di Arcetri.
}

Corresponding author email: andrea.meneghin@inaf.it

Keywords: EURO-CARES; sample curation facility; planetary protection.

EURO-CARES (European Curation of Astromaterials Returned from Exploration of Space) was a threeyear (2015-2017) multinational project funded under the EC's Horizon 2020 research programme to develop a roadmap for a European Extra-terrestrial Sample Curation Facility (ESCF). The project involved 6 different countries and 14 different institutions. The objective was to roadmap a European Sample Curation Facility (ESCF) that would be suitable for the curation of material returned from the Moon, Mars, asteroids and other celestial bodies. While there have been previous studies specific to particular missions, countries, or target bodies, this was the first project to bring together scientists and engineers from across Europe to plan a single facility that would fit the needs of European sample return missions over the next decades. We planned the pathway of our returned samples from the landing site and transport to the facility, to their early and preliminary examination and long-term storage.

With this broad remit, we chose keywords for our ESCF: Flexible, Adaptable and Modular, to guide our planning. This would allow the ESCF to adapt to a wide variety of missions with samples of different sizes and forms, from restricted or unrestricted missions.

The project concentrates on the following main themes:

- Long-term curation of samples;

- Forward and backward planetary protection;

- Definition of a list of analogue samples;

- ESCF infrastructure requirements;

- Techniques and procedures for sample handling and delivery, from landing site to external laboratories;

- Scientific devices and procedures for preliminary examination;

- Life detection and biohazard assessment.

In this work, the main results of the study concerning the conceptual design of the ESCF and the procedures/ workflow (samples, personnel, activities) to be performed inside of such a unique curation facility for both restricted and unrestricted areas are presented. 


\title{
A luminescence In-Situ instrument for planetary dating applications
}

\author{
Coccaro F. ${ }^{1}$, Filippone R. ${ }^{1}$, De Angelis G. ${ }^{1}$ \& Di Iorio A. ${ }^{1}$ \\ ${ }^{1}$ Alma sistemi SRL.
}

Corresponding author email: fco@alma-sistemi.com

Keywords: Luminescence; on-site dating; Martian analogues.

As the ongoing robotic exploration has made some astonishing discoveries, the next major step [1] is to retrieve samples from the Martian surface so they can be analysed in detail in terrestrial laboratories. However, considering the huge costs associated to those missions, an on-site dating of rock and sediment samples allows performing quick tests and select meaningful samples to bring back enabling a cost-effective mission's approach.

Chronological dating of recent events on Mars is problematic as the uncertainties associated with the current methodology (crater counting) are comparable to the younger ages obtained ( 1 Million years). The instrument described is a miniaturized, portable tool for in-situ examination and assessment based on the luminescence method. It is a standard technique with many successful applications $[2,3,4]$. The feasibility of a luminescence-based approach to dating of soil simulants and sediments analogues to Martian deposits has been showed [5]. To minimized weight and power consumption, new techniques and procedures that involves no heater are under development. Different set of LEDs, in blue, green, IR and UV spectra are used to excited different electrons traps to allow more flexibility and used of the better suited techniques depending on the samples under study. Using an X-ray source, the correct dosimetry is estimated. Thanks to the development of its innovative technology, and in addition to planetary exploration application, the instrument will be also suitable for terrestrial field applications as a lightweight and portable dating and analysis instrument for geology and archaeology as well as a risk assessment tool for accident and emergency dosimetry and nuclear mass-casualty events.

\section{References:}

[1] Johnson (2018) - MEPAG.

[2] Wintle (2014) - Treatise on Geochemistry.

[3] Duller (2015) - Springer.

[4] Richter et al. (2017) - Nature.

[5] Detschel et al. (2009) - Journal of Luminescence. 


\title{
Reflectance and emissivity measurements on hydrated salts: a tool to dive into icy planets
}

\author{
Fastelli M. ${ }^{1}$, Comodi P. ${ }^{1}$, Zucchini A. ${ }^{1}$, Maturilli A. ${ }^{2}$, Balic-Zunic T. ${ }^{3} \&$ Rossi M. ${ }^{4}$ \\ ${ }^{1}$ Dipartimento di di Fisica e Geologia, University of Perugia, Italy. \\ 2 Planetary Emissivity Laboratory, DLR German Aerospace Center, Berlin, Germany. \\ ${ }^{3}$ Department of Geosciences and Natural Resource Management, University of Copenaghen, Denmark. \\ ${ }^{4}$ Dipartimento di Scienze della Terra, University of Napoli Federico II, Italy.
}

Corresponding author email: maximiliano.fastelli@gmail.com

Keywords: Reflectance; Emissivity; hydrated salts.

During the Galileo mission, the Near-Infrared Mapping Spectrometer (NIMS) captured more than 70 observations of Europa's surface. The composition of Europa's surface is assumed to be a mixture of nonwater ice materials, i.e. also called dirty ice, where water is stored into crystal structures, such as sulfuric acid hydrates, brines, and salt hydrates. One way to describe in detail the surface ice composition is through the accurate analyses of existing spacecraft data and telescopic observations. However, the library data to compare with planetary observations are usually restricted to small spectral ranges and collected only at room temperature. In this study, the emissivity and reflectance spectra of a selected representative group of minerals (sulphates, chlorides, alkaline-earth or alkaline sulphates and mixed salts) were collected at low $\left(-80 \mathrm{C}^{\circ}\right)$ and high $\mathrm{T}\left(500 \mathrm{C}^{\circ}\right)$. Fundamental S-O banding and stretching vibration absorption bands occur in the 4-5, and 7-10.4 $\mu \mathrm{m}$ regions. The presence of $1.9 \mu \mathrm{m}$ band indicates molecular $\mathrm{H} 2 \mathrm{O}$ and the presence of $1.4 \mu \mathrm{m}$ band indicates that $\mathrm{OH}$ is present. Cation substitution can affect the wavelength position of $\mathrm{SO} 4$ absorption bands. Spectra at high temperatures (HT) give information about dehydration of the minerals and possible phase transitions. Emissivity measurements give us information about $v 3$ absorption band in the spectral range from 600 to $3500 \mathrm{~cm}-1$. Anhydrous sulphates show absorption band in the $1050-1250 \mathrm{~cm}-1$ range corresponding to symmetric stretch of SO4. Hydrated sulphates have absorption band in the $\sim 2500-2700 \mathrm{~cm}-1$ range probably due to the presence of water molecules. The absorption bands characteristic of the non-water ice materials are present in the areas over $5 \mu \mathrm{m}$ where the current data from remote sensing were not collected. For the future missions it is desirable to use instruments able to collect spectra with a range up to $25 \mu \mathrm{m}$. 


\title{
Laboratory activities in support to the Ma_MISS experiment onboard the ExoMars2020 rover
}

\author{
Ferrari M. ${ }^{1}$, De Angelis S. ${ }^{1}$, De Sanctis M.C. ${ }^{1}$, Altieri F. ${ }^{1}$ \& Ammannito E. ${ }^{2}$ \\ ${ }^{1}$ INAF-IAPS Istituto di Astrofisica e Planetologia Spaziali, Via del Fosso del Cavaliere 100, 00133, Roma. \\ ${ }^{2}$ ASI - Agenzia Spaziale Italiana, Via del Politecnico, 00133, Roma.
}

Corresponding author email: marco.ferrari@inaf.it

Keywords: Ma_MISS; subsurface composition; Mars

Ma_MISS (Mars Multispectral Imager for Subsurface Studies) is the Visible and Near Infrared (0.4-2.2 $\mu \mathrm{m})$ miniaturized spectrometer hosted in the drill system of the ExoMars 2020 rover [1] that will characterize the mineralogy and stratigraphy of the excavated borehole wall at different depths $(\leq 2 \mathrm{~m})$ [2]. Ma_MISS is designed to accomplish the following scientific objectives: (1) determine the composition of the subsurface materials; (2) map the distribution of the subsurface $\mathrm{H} 2 \mathrm{O}$ and volatiles; (3) characterize important optical and physical properties of the materials (e.g., grain size); (4) produce a stratigraphic column that will provide information on the subsurface geology.

Previous remote sensing measurements performed by OMEGA and CRISM suggest that the exposed rocks on the surface of the selected landing site (i.e. Oxia Planum) experienced an intense aqueous alteration [3]. The widespread presence of $\mathrm{Fe} / \mathrm{Mg}$ phyllosilicates, and more generally the presence of $\mathrm{OH}$-bearing silicates confirms the interaction between water and the parent rocks. In this framework we perform several spectroscopic measurements of $\mathrm{Fe}, \mathrm{Mg}$ and $\mathrm{Al}$ clays, sulphates, zeolites and hydroxides at low pressure condition.

Infrared reflectance spectra of the selected analog minerals in the range of the Ma_MISS spectrometer show the typical hydration features due to structural $\mathrm{OH}$, bound $\mathrm{H} 2 \mathrm{O}$, and adsorbed $\mathrm{H}_{2} \mathrm{O}$. The spectral response of these hydration features is highly dependent on the sample environment and on the nature of the $\mathrm{H}_{2} \mathrm{O} / \mathrm{OH}$ in the minerals. The behavior of the hydration features near $1.4 \mu \mathrm{m}, 1.9 \mu \mathrm{m}$, and $2.2 \mu \mathrm{m}$, are reported in spectra measured under a Mars-like atmospheric environment.

We are currently working on an environmental chamber that allows analyzing the Martian analogues at low temperature with the aim to simulate the permafrost condition that most likely Ma_MISS will investigate in the Martian subsurface.

References:

[1] Vago et al. (2017) - Astrobiology, 17, 6, 7.

[2] De Sanctis et al. (2017) - Astrobiology, 17, 6, 7.

[3] Quantin et al (2016) - \#2863, 47th LPSC, Houston, TX. 


\title{
The European contribution to Mars Sample Return mission as key opportunity for planetary science
}

\author{
Ferri A. ${ }^{1}$, Cassi C. ${ }^{1}$, Massobrio F. ${ }^{1}$, Centuori S. ${ }^{2} \&$ Pellacani A. ${ }^{3}$ \\ ${ }^{1}$ TAS in ITALY; O. Sutherland, (ESA). \\ ${ }^{2}$ DEIMOS (TBC). \\ ${ }^{3} \mathrm{GMV}, \mathrm{TBC}$.
}

Corresponding author email: antonella.ferri@thalesaleniaspace.com

Keywords: Mars Sample Return; habitability; Martian history.

The physical return of samples from Mars has been a top priority of the international planetary science community for many years. Today, the key science objectives of Mars relate to searches for evidence of past life and constraining past habitability, surveying the history of Martian surface processes and surface materials, understanding the planetary evolution of Mars and its atmosphere, and preparation for potential future human exploration. A wide consensus is held that a mission which enables the analysis on Earth of samples returned from well-characterised sites would allow the greatest science return to be made across these objectives. Previous missions to Mars have been able to make significant progress through in-situ measurements alone, but there is a limit to the science they can perform due to difficulties in miniaturising analytical tools to fit within their payload's capacity. The return of samples allows for analysis on Earth using large, state-of-the-art instrumentation able to exploit advances to technology as they are made. Recent accomplishments in Mars exploration have increased confidence in the technical feasibility of returning samples. NASA and ESA are currently collaborating to study options for an international MSR campaign, capable of delivering a variety of atmospheric and soil samples collected on Mars for analysis on Earth. This international campaign would comprise three missions with the goal of returning samples before the end of 2031. NASA's Mars 2020 rover will perform sampling and caching of samples. The next two missions entail a landing platform, with a fetch rover for collecting the samples left by M2020 and a rocket to launch them into Mars orbit, and a dedicated orbiter spacecraft capable of capturing the orbiting sample and performing a safe transfer and return to Earth. The MSR Earth Return Orbiter and the Sample Fetch Rover have been identified by Europe as possible contributions to the international campaign. 


\title{
Space weathering effects on V-type asteroids
}

\author{
Fulvio D. ${ }^{1}$, Ieva S. ${ }^{2}$, Perna D. ${ }^{2}$, Mazzotta Epifani E. ${ }^{2}$, Strazzulla G. ${ }^{3} \&$ Dotto E. ${ }^{2}$ \\ ${ }^{1}$ Departamento de Fisica, Pontificia Universidade Catolica do Rio de Janeiro, RJ, Brazil. \\ ${ }^{2}$ INAF-Osservatorio Astronomico di Roma, Roma, Italy. \\ ${ }^{3}$ INAF-Osservatorio Astrofisico di Catania, Catania, Italy.
}

Corresponding author email: dfulvio@puc-rio.br

Keywords: V-Type asteroids; space weathering; eucrite.

In recent years, several tens of asteroids have been found to show a surface composition similar to that of Vesta ('V-types'). Many of these objects belong to the Vesta's dynamical family ('Vestoids') although the discovery of V-types outside Vesta's dynamical family opened several questions on the relationship between Vesta, the V-types, and the Howardite-Eucrite-Diogenite meteorites (HEDs). In this talk, we will discuss the result of ion irradiation experiments performed on different samples of eucrites, to simulate the effects of space weathering processes on V-types [1]. We will show that space weathering may explain the whole range of spectral slopes observed for all V-types, except for the Near-Earth Asteroids (NEAs) [2,3]. For these objects, only moderate space weathering effects are evidenced. The astrophysical implications of this puzzling result will be discussed $[2,4]$.

\section{References:}

[1] Fulvio et al. (2012) - Astronomy and Astrophysics 2012, Vol. 537, Letter 11.

[2] Fulvio et al. (2016) - MNRAS, Vol. 455, Issue 1, pp. 584-595.

[3] Fulvio (2018) - Planetary and Space Science, Vol. 164, pp. 37-43.

[4] Ieva (2018) - MNRAS, Vol. 479, pp. 2607-2614. 


\title{
Electrical chondrite characterization for the radar propagation study in planetary crusts
}

\author{
Gabbai F. ${ }^{1}$, Lauro S.E. ${ }^{1}$, Cosciotti B. ${ }^{1}$, Di Paolo F. ${ }^{1}$, Mattei E. ${ }^{1}$, Pettinelli E. ${ }^{1}$, Pratesi G. ${ }^{2} \&$ Carli C. ${ }^{3}$ \\ ${ }^{1}$ Dipartimento di Matematica e Fisica, Università degli Studi Roma Tre, Via della Vasca Navale, 00146 Roma, Italia. \\ ${ }^{2}$ Dipartimento di Scienze della Terra, Università degli Studi di Firenze, Via Giorgio La Pira 4, 50121 Firenze - Italia. \\ ${ }^{3}$ IAPS, Via Fosse del Cavalieri, Roma, Italia.
}

\section{Corresponding author email: f.gabbai@gmail.com}

Keywords: GPR; electromagnetic properties; chondritic meteorites.

Ground Penetrating Radar (GPR) is a geophysical terrestrial exploration method that has recently become one of the most promising technique for planetary subsurface exploration. The capability of GPR to sound planetary bodies relies on the electromagnetic properties of the constitutive materials. Chondritic meteorites and their icy mixtures are considered to be reasonable analogs for cometary material as well as constituent of shallow part of some Galilean Jovian satellite crusts. The knowledge of electromagnetic properties of meteorites allows to estimate the radar response in terms of signal velocity and attenuation. In this work we measured the real and imaginary parts of permittivity of five terrestrial rocks (basalt and andesite) and a L-chondrite meteorite as a function of frequency $(20 \mathrm{~Hz}-1 \mathrm{MHz})$ by using a capacitive cell connected to a selfbalancing bridge. The samples were cut into sheets a few millimeters thick and of about $10 \mathrm{~cm}$ in diameter. Moving the electrodes ( $2 \mathrm{~cm}$ in diameter) on the surface, we studied the spatial variability of dielectric properties of the sample. Terrestrial rocks do not show any significant inhomogeneities at the scale of our set-up, whereas meteorite exhibits a differentiated structure where a core is recognizable surrounded by a less dense material. In general the meteorite sample shows a stronger dispersive behavior compared to terrestrial rocks with higher values for both real and imaginary part of permittivity. In particular, the occurrence of very small grains $(<10$ micrometers) of metal and sulfides in some areas of the meteorite seems to be the cause of such behavior. 


\title{
Laboratory measurements on decomposition of White Soft Minerals (WSM) under conditions of interest to planetology
}

\author{
Mancarella F. ${ }^{1,2}$, D’Elia M. ${ }^{1}$, Orofino V. ${ }^{1}$, Micca Longo G. ${ }^{3}$ \& Longo S. ${ }^{3,4,5}$ \\ ${ }^{1}$ Department of Mathematics and Physics Ennio De Giorgi, University of Salento, Lecce, Italy. \\ ${ }^{2}$ INAF- Istituto di Astrofisica e Planetologia Spaziali, Via del Fosso del Cavaliere, Roma, I-00133, Italy. \\ ${ }_{3}^{3}$ Department of Chemistry, Universita degli studi di Bari Aldo Moro, Via Orabona 4, I-70126 Bari (Italy). \\ ${ }^{4}$ CNR-Nanotec, via Amendola 122/D, Bari 70126, Italy. \\ ${ }^{5}$ INAF-Osservatorio Astrofisico di Arcetri, Largo E. Fermi 5, Firenze I-50125, Italy.
}

Corresponding author email: francesca.mancarella@le.infn.it

Keywords: White Soft Minerals; organic matter; thermal processes.

In the last years the importance of studies on life-related molecules and of the delivery of organic matter to Earth's surface from Space through biosphere was pointed out [1,2]. This kind of molecules, which are very thermolabile, are always associated to solid grains mainly composed by a group of minerals provisionally called White Soft Minerals (WSMs) [3]. WSMs include minerals belonging to the groups of carbonates, like calcite, magnesite, sulphates and many other phases. All the WSMs share promising features related to the possible thermal shield for the chemical decomposition of life-related molecules during the passage through the Earth's atmosphere. Moreover, carbonates are well known for their connection to organic molecules in cometary dust particles and are investigated in the context of planetology [4-10].

In this work, we experimentally illustrate how grains, composed by such kind of minerals, decompose when subject to a thermal process similar to that experienced by mineral grains during atmospheric entry. Several laboratory measurements of gravimetry and spectroscopy are presented and compared to results from theoretical treatment of the thermal process experienced by the samples.

\section{References:}

[1] Bisceglia et al. (2017) - Int. J. Astrobiol. 16, 130-136.

[2] Micca Longo \& Longo (2017) - Int. J. Astrobiol. 16, 368-378.

[3] Micca Longo et al. (2019) - In preparation.

[4] Borg et al. (1999) - Science, 286, 90-94.

[5] Orofino et al. (2000) - Planet. Space Sci. 48, 1341-1347.

[6] Fonti et al. (2001) - JGR 106, 27815-27822.

[7] Rivkin et al. (2006) - Icarus 185, 563-567.

[8] Busemann et al. (2009) - Earth Planet. Sci. Lett. 288, 44-57.

[9] Orofino et al. (2009) - Planet. Space Sci. 57, 632-639.

[10] D'Elia et al. (2017) - Int. J. Astrobiol. 16, 137-142. 


\title{
GAL Hassin, the new center for Outreach of Astronomy and Physical Sciences in Sicily
}

\author{
Masiero S. ${ }^{1}$, Nastasi A. ${ }^{1} \&$ Ziino L. ${ }^{1}$ \\ ${ }^{1}$ Fondazione GAL Hassin-Centro Internazionale per le Scienze Astronomiche, Isnello (PA).
}

Corresponding author email: sabrina.masiero.galhassin@gmail.com

Keywords: GAL Hassin, Madonie, wide field telescope.

The Sky of the Madonie in Sicily is a very important environmental resource. The Astronomical Park GAL Hassin- International Center for Astronomical Sciences -combines frontier scientific research with the teaching and dissemination of Astronomy and Physical Sciences. The GAL Hassin consists of an observational site, located on Mount Mufara at $1865 \mathrm{~m}$, where in the next months a 1-m class remotely controlled wide field telescope (Wide-field Mufara Telescope - WMT) will be placed, and of a structure for educational and outreach activities located nearby to the town of Isnello (Palermo). The Center hosts permanent exhibition of copies of ancient astronomical instruments in the esternal park, and interactive exhibits and multimedia contents in the Museum. Here, lessons and educational presentations are given to visitors. Ten optical telescopes are housed inside a structure, a sort of dome, that moves along two rails. Also a radiotelescope ( $2.4 \mathrm{~m}$ parabola) can be used for outreach education together with a solar laboratory. The total number of visitors exceeds 20000 persons/year, including school classes and general public. The great attraction is the Planetarium, which hosts meetings and outreach events organized for children, students and general public. The Center is open for school tours during the week, the weekends are dedicated to the general public. For different target groups we pursue different strategies. Ways to communicate and popularise astronomy at the GAL Hassin are still a work in progress. I will describe some of the major strategies we use during the guided tours with different categories of visitors. I will also talk about future synergies withh other partners, such as institutes, astronomical observatories, museums, universities and cultural foundations. 


\title{
Molecular oxygen in the surface of Galilean moons investigated in laboratory
}

\author{
Migliorini A. ${ }^{1}$, Ioppolo S. ${ }^{2}$, Kanuchova Z..$^{3,4}$, Strazzulla G. ${ }^{4}$, Piccioni G. ${ }^{1}$ \& Tosi F. ${ }^{1}$ \\ ${ }^{1}$ IAPS-INAF, Rome. \\ ${ }^{2}$ Queen Mary University of London. \\ ${ }^{3}$ Astronomical Institute, Slovakia. \\ ${ }^{4}$ INAF, Observatory of Catania.
}

Corresponding author email: alessandra.migliorini@inaf.it

Keywords: Molecular oxygen; Galilean Moons; MAJIS.

Spectroscopy in the visible range of the Galilean satellites is a suitable way to investigate the surface properties of these objects. In recent years, several species, like $\mathrm{O} 2, \mathrm{O} 3$, and $\mathrm{SO} 2$, have been detected on the surfaces of these satellites, which were thought to be completely covered only by water ice. The detection of two peculiar $\mathrm{O} 2$ absorption bands, centred at 557.3 and $627.5 \mathrm{~nm}$, in the Ganymede trailing face (Spencer et al. 1995) and on Europa and Callisto leading faces (Spencer and Calvin, 2002) led to laboratory experiments in order to better constraint the O2 phases trapped in the water ice surface (Vidal et al. 1997). Spectroscopy in the visible range of the Galilean satellites is a suitable way to investigate the surface properties of these objects. In recent years, several species, like $\mathrm{O} 2, \mathrm{O} 3$, and $\mathrm{SO} 2$, have been detected on the surfaces of these satellites, which were thought to be completely covered only by water ice. The detection of two peculiar $\mathrm{O} 2$ absorption bands, centred at 557.3 and $627.5 \mathrm{~nm}$, in the Ganymede trailing face (Spencer et al. 1995) and on Europa and Callisto leading faces (Spencer and Calvin, 2002) led to laboratory experiments in order to better constraint the O2 phases trapped in the water ice surface (Vidal et al. 1997).

New laboratory measurements of solid oxygen and its mixtures with $\mathrm{H} 2 \mathrm{O}, \mathrm{N} 2, \mathrm{CO} 2$, and $\mathrm{NH} 3$ have been carried out using the Open University portable astrochemistry vacuum chamber attached to the ultraviolet vacuum beamline of the synchrotron ASTRID 2 facility at the Aarhus University.

The icy surfaces of the galilean moons were simulated and studied in the 550-650 nm spectral region. The solar irradiation was simulated through irradiation with low energy electrons (Jones et al. 2014).

In this work, we present the preliminary results obtained by comparing the laboratory measurements and ground-based observations of Ganymede and Europa at TNG and the Asiago facilities.

This activity is in support of future observations of the Galilean moons with MAJIS on JUICE, scheduled for launch in 2022.

\section{References:}

[1] Spencer et al. (1995) - J. Geophys. Res.

[2] Spencer and Calvin (2002) - The Astronomical Journal.

[3] Vidal et al. (1997) - Science.

[4] Jones et al. (2014) - The Astrophysical Journal. 


\title{
Intracrystalline geothermometry applied to a Martian analogue
}

\author{
Murri M. ${ }^{1}$, Cámara F. ${ }^{2}$, Adam J. ${ }^{3}$, Domeneghetti M.C. ${ }^{1}$ \& Alvaro M. ${ }^{1}$ \\ ${ }^{1}$ Department of Earth and Environmental Sciences, University of Pavia, Italy. \\ ${ }^{2}$ Department of Earth Sciences ‘A. Desio', University of Milan, Italy. \\ ${ }^{3}$ Department of Earth and Planetary Sciences, Macquarie University, Sydney, 2109, Australia.
}

Corresponding author email: mara.murri01@universitadipavia.it

Keywords: geothermometry, nakhlites, cpx, opx.

The application of intracrystalline "geothermometers" to Martian nakhlite samples is lacking validations for the calibration equations that express the $\operatorname{lnkD}$ as a function of $1 / \mathrm{T}(\mathrm{K})$ for clino- and ortho-pyroxenes (cpxs and opxs). To overcome these limitations, crystals of cpx and opx, grown in rock analogues synthesized under controlled conditions, are needed to compare the closure temperature (TC) of the intracrystalline Fe $2+-\mathrm{Mg}$ ordering process with the quenching temperature (TQ) of the synthesis experiments. Since in these experiments the system remains closed for the entire cooling process the intracrystalline thermometry records the last thermal event experienced by the single mineral phase.

For this purpose, we measured by single crystal X-ray diffraction the Fe2+-Mg order degree (kD) on three pairs of synthetic samples containing both cpx and opx as separated crystals recovered after quenching from high temperature. The site occupancies from X-ray diffraction have been interpreted adopting the calibration equation by [1] for cpx and that by [2] for opx.

Our results demonstrate for the first time the quite remarkable agreement between calculated closure temperature (TC) and actual quenching temperature (TQ) for synthesis products with the largest discrepancy of the order of few tens of degrees. We then applied our geothermometer [1] to natural augite samples from Theo's Flow lava, the terrestrial analogue of Martian nakhlites [3] in order to shed light on its thermal history and magmatic evolution.

\section{References:}

[1] Murri et al. (2016) - Am Mineral 101, 12, 2747-2750.

[2] Stimpfl et al. (2005) - Contrib Mineral Petr 150, 319-334.

[3] Lentz et al. (2011) - Geol Soc Am Spec Pap, 483, 263-277. 


\title{
Experimental petrology and planetary studies: spectroscopy on silicate glasses for the exploration of volcanic terrains
}

\author{
Pisello A. ${ }^{1,2}$, De Sanctis M.C. ${ }^{2}$, Maturilli A. ${ }^{3}$, De Angelis S. ${ }^{2}$, Ferrari M. ${ }^{2}$, Vetere F. ${ }^{1}$, \\ Pauselli C. ${ }^{1} \&$ Perugini D.1 \\ ${ }^{1}$ Department of Physics and Geology, University of Perugia, Piazza Università, 06123 Perugia, Italy. \\ ${ }^{2}$ Istituto di Astrofisica e Planetologia Spaziali, Istituto Nazionale di Astrofisica, Rome, Italy. \\ ${ }^{3}$ Institute for Planetary Research, DLR, Rutherfordstrasse 2, 12489 Berlin-Adlershof, Germany.
}

Corresponding author email: alessandropisello@gmail.com

Keywords: Emissivity; Reflectance; Silicate glasses.

Silicates are the main constituents of terrains on terrestrial planets in the solar system. Silicate glasses represent the amorphous phase of silicate crystals. Typically, volcanic rocks are afanitic or porphyric rather than holocrystalline: the fraction of amorphous material is therefore major than the fraction of crystalline material. Many works focus on the spectral characterization of crystalline silicates, but it is important to also study silicate glasses' spectral properties to better interpret available remotely sensed data from past and future missions.

Emissivity and reflectance spectra have been investigated on different series of silicate glasses, having a wide compositional range representing different magmatic series. Glasses were synthesized starting from natural rocks. Spectral characterization was performed in two different laboratories for two different ranges: i) Planetary Emissivity Laboratory (DLR, Berlin): mid-IR data at different temperatures have been acquired.

Reflectance (fresh and after heating at $700^{\circ} \mathrm{C}$, spectral range $\left.1-18 \mu \mathrm{m}\right)$ and emissivity $\left(150^{\circ} \mathrm{C}, 300^{\circ} \mathrm{C}\right.$, $450^{\circ} \mathrm{C}, 700^{\circ} \mathrm{C}$; spectral range 5-16 $\mu \mathrm{m}$ ) spectra were collected. ii) Institute for Space Astrophysics and Planetology (IAPS-INAF, Rome): near-IR spectra (fresh sample, spectral range 0.35-2.5 $\mu \mathrm{m}$ ) have been acquired in reflectance.

Results show that the shift of the spectra, by taking Christiansen feature (CF) as a reference point, linearly relates with the silica content, the SCFM factor and/or the degree of polymerization state via the NBO/T parameter: the more evolved is the composition, the more polymerized the structure, the shorter the wavelength at which $\mathrm{CF}$ is observable. Short-range order features (Q species) are also distinguishable in the mid-IR range. For what concerns near-IR reflectance, it is observed how the spectra are heavily influenced by iron content, iron speciation (Fe III/ Fe tot) and by occurrence of magnetite nanolites. 


\title{
Laboratory study in support of OSIRIS-REx mission. Mineral and organics reflectance spectra in space simulated environment as hint for a deep investigation of Bennu surface
}

\author{
Poggiali G. ${ }^{1,2}$, Brucato J.R. ${ }^{2}$, Corazzi M.A. ${ }^{1,2}$, Meneghin A. ${ }^{2}$, Ieva S. ${ }^{3}$, Dotto E. ${ }^{3}$, \\ Mazzotta Epifani E. ${ }^{3} \&$ Fornaro T. ${ }^{4}$ \\ ${ }^{1}$ Università degli Studi di Firenze, Dipartimento di Fisica ed Astronomia, Sesto Fiorentino (FI), Italy. \\ ${ }^{2}$ INAF - Osservatorio Astrofisico di Arcetri, Firenze, Italy. \\ ${ }^{3}$ INAF - Osservatorio Astronomico di Roma, Monte Porzio Catone, Rome, Italy. \\ ${ }^{4}$ Carnegie Institution for Science, Geophysical Laboratory (GL), Washington DC, USA.
}

Corresponding author email: giovanni.poggiali@unifi.it

Keywords: OSIRIS-Rex; Infared spectroscopy; Bennu.

Bennu, the target of NASA OSIRIS-REx space mission, is a Near Earth Asteroid (NEA) classified as a carbonaceous B-type asteroid. This class of NEAs is thought to be the most primitive bodies in the Solar System directly linked with planetary formation and the transfer of prebiotic molecules on Earth. From ground-based observations the surface of carbonaceous asteroids shows spectral features linked with the presence of carbon, while space missions have revealed the presence of organic material on the rocky surfaces of these bodies. For this reason, in the next future, the detection of organic material on Bennu will be the most awaited result for the OSIRIS-REx mission. Since the approach and the rendezvous phase, started on December 3rd, Bennu is studied in detail with the two spectrometers on board of the spacecraft: OVIRS in the visible (VIS) and nearinfrared (NIR) range and OTES for mid-infrared (MIR).

The experimental work here presented will show a wide laboratory study on infrared spectrometry of mineral and organics in asteroidal space simulated condition. The aim of the study is the investigation on how the presence of different mineral or rocks can influence the survival and destruction of simple and complex organic material in airless bodies affected by UV irradiation. Laboratory spectra measured in space simulated conditions (i.e. vacuum and low temperature) in a wide wavelength range from VIS to FIR could be in the future directly compared with data from the spacecraft, helping the interpretation of spectroscopic features present on Bennu' surface. Detailed studies performed in laboratory, with the support of model analysis, are an effective tool to increase our knowledge on nature and history of small bodies in the Solar System. 


\title{
Experimental set up used to characterize the optical properties of gases at typical planetary conditions
}

\author{
Stefani S. ${ }^{1}$, Piccioni G. ${ }^{1} \&$ Snels M. ${ }^{2}$ \\ ${ }^{1}$ IAPS-INAF, via del fosso del cavaliere 100, 00133 Rome Italy. \\ ${ }^{2}$ ISAC-CNR, via del fosso del cavaliere 100, 00133 Rome Italy.
}

Corresponding author email: stefania.stefani@inaf.it

Keywords: gas optical properties, pressure, temperature.

The laboratory studies of the optical properties of gases at typical planetary conditions, high/low pressure and high/low temperature, are of major importance to implement the input parameters of the radiative transfer models. Furthermore, the experimental data, will support the study of our solar system bodies and will also contribute significantly to the understanding of the exoplanets which, in great part of the cases explored until now, present conditions most different from those terrestrial. For this purpose, we propose a set of different experimental set up able to measure the optical properties of gases, like as $\mathrm{CO}_{2}, \mathrm{CH}_{4}, \mathrm{H}_{2}, \mathrm{H}_{2}+\mathrm{He}$ and $\mathrm{H}_{2}+\mathrm{He}+\mathrm{CH}_{4}$, at low/high pressure and low/high temperature. The experimental set-up is composed by two dedicated gas cells integrated with a Fourier Transform InfraRed (FT-IR) spectrometer. One cell, a High Pressure High Temperature (HP-HT) gas cell, is designed to sustain pressures up to $200 \mathrm{bar}$, temperatures up to $570 \mathrm{~K}$ and it is characterized by an optical path of about $2 \mathrm{~cm}$. Another cell, a Multi Pass (MP), has a variable optical path pre-settable from 2.5 to $30 \mathrm{~m}$, able to work with gases at pressure up to 10 bar and temperature up to $600 \mathrm{~K}$. In order to study the very weak absorptions (about 10-10) found, for example in the so called "atmospheric windows", we realized a second independent experimental set up which takes advantage of the Cavity Ring Down (CRD) technique. This apparatus allows to achieve effective optical paths of several kilometers by using resonant optical cavities, equipped with highly reflective mirrors $(99,999 \%)$ and is able to work with pressure up to 50 bar and temperatures up to about $370 \mathrm{~K}$. A new setup with extended characteristics in temperature and pressure range is under construction to overcome some present limitations and here is briefly discussed as well. The new innovative apparatus will be suitable for both FT-IR and Cavity Ring Down (CRD) techniques, sustaining gases at pressure from a few mbars to 50 bar and temperature from $75 \mathrm{~K}$ up to $520 \mathrm{~K}$. 


\title{
Ion and electron bombardment of materials studied in the laboratory in support of planetary space missions
}

\author{
Strazzulla G.
}

INAF-Osservatorio Astrofisico di Catania, Italy.

Corresponding author email: Giovanni.strazzulla@inaf.it

Keywords: energetic ion; electron bombardment; space weathering.

The study of the effects induced by energetic ions and electrons bombarding frozen gases or solid silicates and carbon based materials have been investigated since several years with a view to their relevance to understand the evolution of physical and chemical properties of the surfaces of airless bodies in the Solar System.

The results contribute to understand the results obtained by instruments placed at Earth or in space and to properly plan new observations.

Here I will present some results recently obtained in laboratories placed in Caen (France), Aarhus (Denmark) and Catania (Italy). The results are discussed in the light of their relevance in support of space missions, in particular Bepi-Colombo (ESA, JAXA), JUICE (ESA) and JWST (NASA). 


\title{
The use of a quantum filter in astronomical imaging
}

\author{
Strumia F. ${ }^{1}$, Brez A. ${ }^{2}$, Bacci P. ${ }^{3} \&$ Paolicchi P. ${ }^{1}$ \\ ${ }^{1}$ Dipartimento di Fisica, Università di Pisa. \\ ${ }^{2}$ INFN, Sezione di Pisa. \\ ${ }^{3}$ GAMP (Gruppo Astrofili Montagna Pistoiese.
}

Corresponding author email: paolo.paolicchi@unipi.it

Keywords: quantum filter, image contrast, bright spectra.

A filter made of an array of equidistant holes is described. It can allow to improve the image contrast and to superimpose bright spectra to the usual images. A few preliminary tests have been performed, and a more thorough testing and analysis is required to qualify the potential of the method and define its applications. 


\section{Authors' Index}

Authors are listed alphabetically: For each contribution, the page number and the session are given.

Aboudan A.

Adam J.

Adriani A.

Affer L.

Alei E.

Aloisi F.

Altavilla G.

Altieri F.

$52,54,57,75,79,91,92$

Altieri G.

Alvaro M.

Ammannito E.

Anselmo L.

Antoniucci S.

Ardizzone F.

Arnold G.

Aronica A.

Arzilli F.

Ascione G.

Asimow P.D.

Bacci P.

Baioni D.

Balbi A.

Balic-Zunic T.

Balucani N.

Barbaro A.

Barbieri M.

Barghini D.

Bariselli F.

Baroni C.

Barros Dias B.R.

Barucci M.A.

Bassan M.

Battistuzzi M.

Beck P.

Bellesi M.

Bellucci G.

Benatti S.

Bennett C.

Bernardi F.

Berrilli F.

Bertini I.

Bertoli S.

Bettanini C.

Bhandari N.

Bianco G.

Bierhaus E.B.

Bignamini A.

Billi D.
93,149

128,156

$51,52,79,80,91$

$18,19,29,33,37$

69

96

81

125,149

102

115

56

$112,123,124$

73,78

141

142

137

161

53

35,38

148

36

128

120

129,140

144

55

144

112

102

37

123

130

54,81

$20,21,23,29,45$

121

97

41

100, 116

55

59

140

98

121

21, 29

38
Bindi L.

137

Bockelee-Morvan D.

112,124

Bolton S.

51,80

Bonadiman C.

131, 139

Bonnarel F.

29

Bonomo A.

26

Brez A.

161

Brucato J.

Brucato J.R.

Brunetti M.T.

Brunetto R.

Burke K.

Buzzoni A.

Cagnazzo C.

$39,115,121,146,158$

$56,66,118$

135,138

121

96, 98

Cámara F.

41

156

Campins H.

121

Camplone V.

57

Campo V.

85

Capaccioni F. 11, 31, 59, 92, 93, 112,

123,124

Cappuccio P.

$60,63,64$

Capretti S.

69

Capria M.T.

$22,27,59,70,88,89$,

$94,112,124,125$

Capuzzo-Dolcetta R.

Caracciolo A.

$36,129,132$

Cardinale $\mathrm{M}$.

Carli C.

$58,71,75,85,92,93,133$,

$134,143,152$

Carrozzo F.G. $\quad 54,57,81,111,125$

Carta R.

Cartacci M.

82

Casavecchia P.

36

Cassi C. 150

Cecchi Pestellini C. 19

Centuori S. $\quad 150$

Cheng Y.-C. $\quad 124$

Chiarini M. $\quad 97$

Chojnacki M. 90

Ciaravella A. 19

Ciarcia S. 63

Ciarniello M. 111, 112, 123, 124, 125

Cibin L. $\quad 97$

Cicchetti A. 52, $59,82,91$

Cicconi M.R. $\quad 141$

Claeys P. $\quad 144$

Claudi R. $\quad 18,19,21,23,37$

Coccaro F. $\quad 147$

Cocola L. $\quad 37$ 
Collu C.

Colombetti P.

66

Comodi P.

Coradini M.

140

148

Corazzi M.A.

82

Cosciotti B.

39,158

82,152

Cremonese G. 57, 59, 74, 84, 88, 93, 118

Cruciani G.

139

D'Achille G.

D'Aversa E.

D'Elia M.

d'Hendecourt L.

D'Orefice M.

Da Deppo V.

Daerden F.

Dall'Oro A.

Daly M.

Damasso M.

De Angelis E.

De Angelis G.

De Angelis I.

De Angelis S.

De Marchi F.

De Sanctis M.C.

$59,70,111,112,114$, $120,123,124,125,134$,

149,157

De Toffoli B.

84

Debaille V.

144

Debei S.

59

Delbo M.

Dell'Oro A.

113,122

Della Corte V.

$27,117,126,135,138$

Della Giustina D.

121

Depiesse C.

54,81

Deshapriya P.

124

Desidera S.

Di Achille G.

Di Benedetto $\mathrm{M}$.

$60,61,62$

60,63

Di Cecco A.

Di Cintio P.

98

99

147

Di Iorio A.

Di Paola A.

98

Di Paolo F.

82,152

Di Pietro I.

Di Stefano I.

Dinelli B.M.

Dionnet Z.

Dirri F.

Domeneghetti M.C.

Doose L.

Dotto E.

Durante D.

Elder C.
Erard S.

112,124

Esposito F.

90

EURO-CARES Team

Faggi S.

146

Falvella M.C.

Fastelli M.

40

98

148

Fedele D.

24,32

Federico C.

114, 124

Fenucci M.

101

Ferranti L.

$67,71,72,85$

Ferrari M.

$134,149,157$

Ferrari S.

85

Ferri A.

150

Fierro D.

98

Figer A.

96

Filacchione G. 59, 91, 112, 123, 124

Filippone R. $\quad 147$

Fink U. 124

Fiorenza E. $\quad 106$

Fioretti A.M. 128

Fiorucci S. $\quad 66$

Flamini E. $\quad 82$

Folco L. $\quad 135,138,141,143$

Fonte S. $\quad 125,32$

Formisano M. $\quad 112,114,117,124$

Fornaro T. 158

Fornasier S. 112

Franceschelli M. 139

Frigeri A. $\quad 125$

Fulle M. $\quad 49$

Fulvio D. $\quad 151$

Gabbai F. $\quad 152$

Galleti S. $\quad 96$

Galliani M. $\quad 16$

Galluzzi V. $\quad 67,70,71,74,85$

Galuzzo D. $\quad 14,41,129,136,140,142$

Garrido A. 25

Gatti L. $\quad 70$

Gemelli M. $\quad 135,138$

Genova A. $\quad 68$

Ghiara M.R. $\quad 142$

Giacobbe P. $\quad 26$

Giacomini L. $\quad 16,58,67,69,70,71$,

72,93

Giardino M. $\quad 125,94$

Giovannelli L. 41

Giuli G. $\quad 130,141,144$

Glass B.P. $\quad 141$

Goderis S. $\quad 144$

Golubov O. 119

Goodrich C. $\quad 128$

Graciotti R. 85

Grassi D. $\quad 52,79,91$ 
Guarcello M.

Guichard J.

Guzzetta L.

Guzzetti F.

HADES collaboration

Helber B.

Hiesinger $\mathrm{H}$.

HIRES Consortium

$\mathrm{Hu}$ J.

Iacovone D.

Iafolla $\mathrm{V}$.

Iess L.

Ieva $\mathrm{S}$.

Ioppolo S.

Istiqomah I.

Ivanovski S.L.

JIRAM team

Juno team

Kanuchova Z.

Kappel D.

Kartalev M.

Kaspi Y.

Kazakov A.

King A.

Kling A.

Knezevic Z.

Koeberl C.

Kovacevic A.B.

Kugel U.

La Forgia $F$.

La Rocca N.

Lanzafame G.

Lari G.

Lauretta D.S.

Lauro S.E.

Lazzarin M.

Lazzaro D.

Lazzarotto F.

Lazzoni C.

Lefevre C.

Lenzi S.

Lepore G.O.

Leyrat $\mathrm{C}$.

LICIACube team

Lippi M.

Litti L.

Locci D.

Longo S.

Longobardo A.

Longobardo A.

Lopez-Moreno J.-J.
98

96

67,72

56

25

144

92

43

137

98

106

63,65

$100,115,151,158$

155

123

27, 49, 70, 73, 78,

117,126

52,79

51

155

$112,123,124$

73

63

73, 78

135

84

122

141,144

35

97

116,120

37

131

101, 107

121

82,152

$100,116,120$

120

73,78

28

106

48, 86

144

112,124

103

40

128

19,45

42,153

111

$112,117,124,125$,

135,138

54,81
Lorenzoni L.

83

Louys M.

29

Lucchesi D.M.

102,106

Lucchetti A.

Lucente M.

$70,74,84,118$

Lugari C.

106

Luzzi E.

131,139

$\mathrm{Ma}$ C.

75

137

Maccone C. $\quad 76$

Maggio A. 25

Magin T.

144

Magnafico C.

102,106

Magni G.

Malaspina M.

Maldonado J.

$114,124,31$

16

25

Mancarella F.

153

Mancinelli P.

Mancini L.

131,141

Mancuso S.

140

Mangano V.

73,78

Mann R.

63

Manzari P. $\quad 134$

Maraviglia A. $\quad 15$

Marchi M. $\quad 139$

Marconi A. $\quad 43$

Margheritis D.B. $\quad 47$

Marinangeli L. $\quad 77,84,85,87$

Marinucci W.

141

Maris M.

Martins Z.

$29,33,46,49$

Marzari F.

44

Marzo C.

28,119

98

Masiero S.

104,154

Mason J.

54,81

Massetti S.

73,78

Massironi M. 59, 67, 71, 74, 84, 85, 92,

$93,118,143$

Massobrio F.

150

Matassoni F.

96

Mattei E.

82,152

Maturilli A.

148,157

Mazzotta Epifani E.

$100,103,115$,

151,158

McKibbin S.J.

144

Meinert $\mathrm{C}$.

Melis M.T.

66,85

Meneghetti M.

128

Meneghin A.

146, 158

Mennella V.

123

Mesa D.

120

Micca Longo G.

42,153

Micela G.

Michel P.

$19,25,45,98$ 
Micheli M.

115

Migliorini A. $\quad 32,52,91,116,120,155$

Milillo A.

$45,73,78,107$

Modica P.

44

Moggi Cecchi V.

$134,142,133$

Molaro J.

121

Molinari S.

32

Molinaro M.

21,29

Mongelluzzo G.

90

Moriconi M.L.

$52,79,91$

Morosinotto T.

Moroz L.V.

37

123

Mottola S.

Mudric T.

88

Mumma M.J.

40

Munaretto G.

74,84

Mura A. $\quad 45,51,52,73,78,79,80,91$

Murana A.

83

Murante G.

Murri M.

Musetti B.

$33,46,48,49$

156

Najorka J.

47

143

Nascimbeni V.

26,29

Nastasi A.

104, 154

Nava J.

Negri B.

Nestola F.

128

Noe Dobrea E.

84

Nolan M.C.

121

Noschese R.

$52,59,91$

Notaro V.

65

Oliva F.

Ori G.G.

$32,54,81$

83

153

Orofino V.

82

Orosei R.

73,78

Orsini S.

OSIRIS-REx Team

118,121

13,30

55,83

Pacifici A.

Paganini L.

Pajola M.

Palazzi E.

Palomba E.

Palomba E.

Palumbo P.

Pantaloni M.

Paolicchi P.

Pardini C.

Patel M.R.

Pauselli C.

Pellacani A.

Pelorosso B.

Penasa L.
Perna D.

Peron R.

$100,105,115,151$

102,106

Perozzi E.

Peruccacci S.

$94,98,100,115$

Perugini D.

Petralia A.

Petropoulou V.

56

157

$19,25,45$

Pettinelli E.

116

Piccioni G.

Pilorget C.

82,152

$52,91,155,159$

123

Piluso E.

77

Pinto L.D.

31

Pirrotta S. $\quad 57$

Pisello A. $\quad 157$

Pittarello L. $\quad 144$

Plainaki C. 52, $\quad 51,94$

PLANMAP Team 93

Poch O. $\quad 123$

Podda S. 66

Poggiali G. $\quad 39,115,158$

Poletto L. $\quad 37$

Politi R. 32, 59

Polychroni D. 27

Pommerol A. $\quad 123$

Pompilio L. $\quad 77,84,87$

Pondrelli M. $\quad 85$

Pozzobon R. 58, 84,85

Pratesi G. $\quad 133,134,141,142,152$

PRISMA collaboration $\quad 136$

Provenzale A. $\quad 46,48,49,86$

Pucacco G. $\quad 102$

Quirico E. 112, 123

Racioppa P. 65

Ragazzoni R. 97

Raponi A. $\quad 111,112,123,125$

Raymond C.A. $\quad 111,125$

Re C. $\quad 59,88$

Rinaldi G. $\quad 112,117,124$

Rispoli R. $\quad 73,78$

Ristic B. $\quad 54,81$

Rizk B. 121

Rognini E. $\quad 111,125,89$

Roma M. 85

Rosetta/VIRTIS Team 112

Rosi M. 36

Rossi A. 99, 119

Rossi A.P. $\quad 75$

Rossi A.P. $\quad 85$

Rossi M. 148, 142

Roszjar J. 144

Rothery D.A. 93

Rotundi A. $\quad 27,117,126,135,138$

Rousseau B. 112,123 
Rubinetti S.

Russell C.T.

Saillenfest M.

Salese F.

Salinari P.

Salvatore M.C.

Sandrelli S.

Santini C.

Santoli F.

Sauro F.

Scaioni M.

Scarciglia F.

Scheeres D.J.

Schisano E.

Schröder S.

Schwartz S.R.

Semenzato A.

Serra D.

Serventi G.

Sessler G.

Sgavetti M.

Shaddad M.H.

Silva L.

Silvestro S.

Simioni E.

Simonetti P.

Sindoni G.

Sinha N.

Skouteris D.

Slemer A.

Snels M.

Soens B.

Soldovieri F.

Sordini R.

Spanò $\mathrm{P}$.

Spiess R.

Spoto F.

Stefani S.

Strazzulla G.
140

111,125

101

62,84

97

55

16

130

106

84

66

77

119

32

111,123

121

74

107

58

97

58

128

$33,46,49$

84,90

$59,84,88$

49

$52,57,91,94$

140

36

59

159

144

82

$52,73,91$

104

143

122

159

$151,155,160$
Strumia F.

161

Suttle M.D.

Taffoni G.

$135,138,143$

Tangari A.C.

Taricco C.

77,84

140

Tassinari R.

139

Teodoro L.

84

Thomas I.R.

54,81

Tombesi F.

Tommei G.

107, 108

Tosi F.

$52,91,111,112,123$,

125,155

Turrini D.

Vaccaro C.

$27,32,33,91$

Valsecchi G.B.

van der Bogert C.H.

Vandaele A.C.

$99,109,115$

Vaz D.A.

92

54,81

$61,62,90$

Verrecchia F.

33

Vetere F.

157

Villanueva G.L.

40

Vincent J-B.

124

Vinogradoff V.

123,134

VIR team.

111

Visco M.

102

Vladilo G.

von Hardenberg J.

Walsh K.

Willame Y.

Wilson J.

Wislocka A.M.

Wright J.

Yamaguchi A.

Zakharov V.V.

Zambon F.

Ziino L.

Zinzi A.

Zucchini A.

Zusi M.
$33,45,46,49$

121

54,81

84

35

93

144

$27,117,126$

$71,92,93,111$

104,154

$33,57,85,88,89,94$

148

59 


\section{CON IL PATROCINIO E IL SUPPORTO DI}

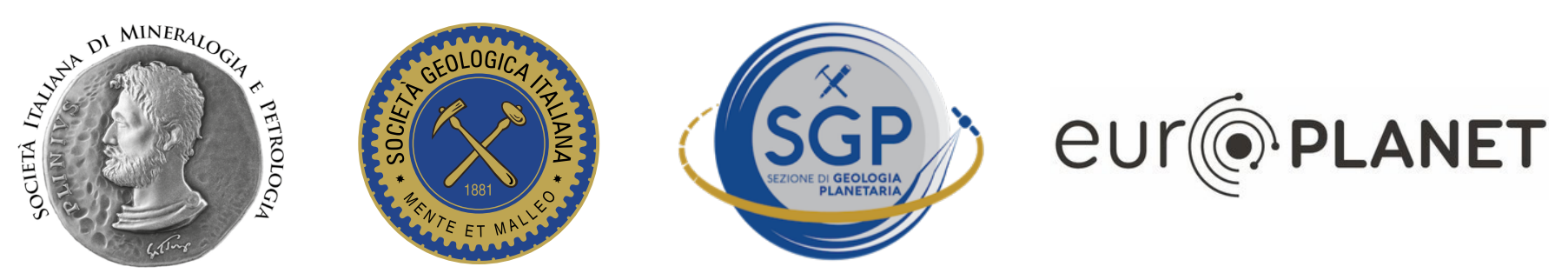

ThalesAlenía

a Thales/Leonardo company SPACe

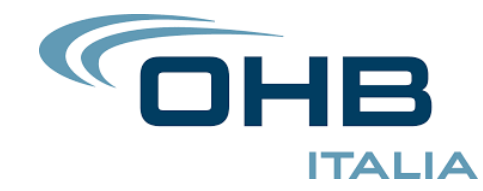

